\title{
High-Energy Neutrino Astrophysics: Status and Perspectives
}

\author{
U.F. Katz ${ }^{\mathrm{a}, 1}$, Ch. Spiering ${ }^{\mathrm{b}, *}$ \\ ${ }^{a}$ ECAP, University of Erlangen, Erwin-Rommel-Str. 1, 91058 Erlangen, Germany \\ ${ }^{b}$ DESY, Platanenallee 6, 15738 Zeuthen, Germany
}

\begin{abstract}
Neutrinos are unique cosmic messengers. Present attempts are directed to extend the window of cosmic neutrino observation from low energies (Sun, supernovae) to much higher energies. The aim is to study the most violent processes in the Universe which accelerate charged particles to highest energies, far beyond the reach of laboratory experiments on Earth. These processes must be accompanied by the emission of neutrinos. Neutrinos are electrically neutral and interact only weakly with ordinary matter; they thus propagate through the Universe without absorption or deflection, pointing back to their origin. Their feeble interaction, however, makes them extremely difficult to detect. The years 2008-2010 have witnessed remarkable steps in developing high energy neutrino telescopes. In 2010, the cubic-kilometre neutrino telescope IceCube at the South Pole has been completed. In the Mediterranean Sea the first-generation neutrino telescope ANTARES takes data since 2008, and efforts are directed towards KM3NeT, a telescope on the scale of several cubic kilometres. The next years will be key years for opening the neutrino window to the high energy Universe. With an instrumented volume of a cubic kilometre, IceCube is entering a region with realistic discovery potential. Discoveries or non-discoveries of IceCube will have a strong impact on the future of the field and possibly mark a "moment of truth". In this review, we discuss the scientific case for neutrino telescopes, describe the detection principle and its implementation in first- and second-generation installations and finally collect the existing physics results and the expectations for future detectors. We conclude with an outlook to alternative detection methods, in particular for neutrinos of extremely high energies.
\end{abstract}

Keywords: Astroparticle physics, Neutrino astronomy, Neutrino telescopes, Neutrino interactions, Cosmic neutrinos, Neutrino oscillations, Acoustic detection, Radio detection PACS: 07.07.Df, 12.60.Jv, 13.15.+g, 13.85.Tp, 14.60.Pq, 91.50.-r, 92.10.-c, 95.30.Cq, 95.35. + d, 95.55.Vj, 95.85.Pw, 95.85.Ry, 98.70.Sa

\footnotetext{
*Email: csspier@ifh.de

${ }^{1}$ Email: katz@physik.uni-erlangen.de
} 


\section{Contents}

1 Introduction 1

2 Scientific Background and Motivation 3

2.1 Neutrinos from cosmic accelerators . . . . . . . . . . . . . . . 3

2.1 .1 Cosmic rays . . . . . . . . . . . . . . . . . 3

2.1.2 Production of neutrinos . . . . . . . . . . . 5

2.1 .3 Galactic sources . . . . . . . . . . . . . . . 6

2.1.4 Extragalactic sources . . . . . . . . . . . . . 8

2.2 Particle physics and exotic phenomena . . . . . . . . . . . . 11

2.2 .1 Indirect search for dark matter . . . . . . . . . . . . . . . 12

2.2 .2 Super-heavy particles . . . . . . . . . . . . . . . . . 13

2.2.3 Neutrino oscillations . . . . . . . . . . . . . . . . . . . . 14

2.3 Environmental and marine sciences . . . . . . . . . . . . . . . 16

3 Detection Principles 18

3.1 Neutrino interactions . . . . . . . . . . . . . . . . . . . . . . 18

3.2 Cherenkov light . . . . . . . . . . . . . . . . . . . . 21

3.3 Light propagation and detection . . . . . . . . . . . . . . . 21

3.4 Detection of muon tracks and cascades . . . . . . . . . . . . . [ 23

3.4 .1 Muon tracks . . . . . . . . . . . . . . . . . . . . 23

3.4 .2 Cascades . . . . . . . . . . . . . . . . 25

3.5 Effective area and sensitivity . . . . . . . . . . . . . . . . 26

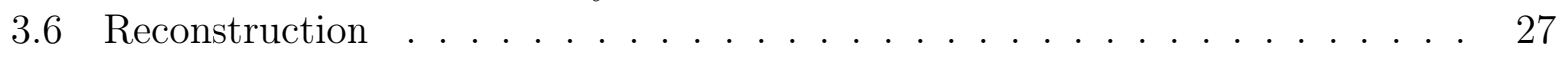

4 First-Generation Neutrino Telescopes

4.1 DUMAND . . . . . . . . . . . . . . . . . . . . . 30

4.2 The Baikal neutrino telescope . . . . . . . . . . . . . . . . 31

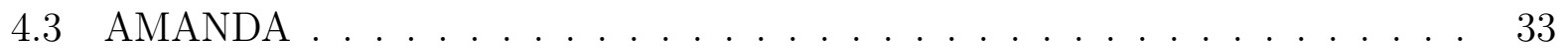

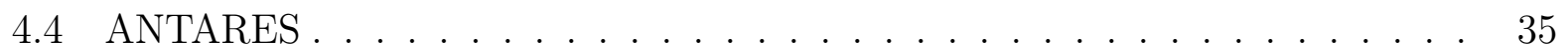

4.5 NEMO . . . . . . . . . . . . . . . . . . . . . . . . . 38

4.6 NESTOR . . . . . . . . . . . . . . . . . . . . . . . . . . 39

5 Second-Generation Neutrino Telescope Projects 40

5.1 IceCube . . . . . . . . . . . . . . . . . . . 40

5.1 .1 DeepCore . . . . . . . . . . . . . . . . . . 42

5.1 .2 IceTop . . . . . . . . . . . . . . . . . . . 43

5.1 .3 IceCube as a supernova burst detector . . . . . . . . . . . . 43

$5.2 \mathrm{KM} 3 \mathrm{NeT} \ldots \ldots \ldots \ldots \ldots \ldots \ldots \ldots$

5.3 GVD in Lake Baikal . . . . . . . . . . . . . . . . . . 46 
6 Physics Results and Perspectives 48

6.1 Atmospheric neutrinos . . . . . . . . . . . . . . . . . . . 48

6.2 Cosmic neutrinos . . . . . . . . . . . . . . . . . 50

6.2.1 Searches for diffuse cosmic neutrino fluxes . . . . . . . . . . . 51

6.2.2 Searches for steady neutrino point sources . . . . . . . . . . 52

6.2.3 Searches for variable neutrino sources . . . . . . . . . . . . . . 57

6.2.4 Alert programs . . . . . . . . . . . . . . . 58 5

6.3 Dark matter and other exotic particles . . . . . . . . . . . . . . . 60

6.3.1 Dark matter . . . . . . . . . . . . . . . . . 60

6.3.2 Magnetic monopoles, Q-balls and nuclearites . . . . . . . . 63

6.4 Cosmic ray physics . . . . . . . . . . . . . . . . . . . 64

7 Alternative Detection Principles for Extreme Energies $\quad 66$

7.1 Detection via air showers . . . . . . . . . . . . . . . . . 66

7.2 Radio detection . . . . . . . . . . . . . . . . . . . . . . . . . . . . . . . . . . . .

7.3 Acoustic detection . . . . . . . . . . . . . . . . . . . .

8 Summary and Outlook $\quad 70$

$\begin{array}{ll}\text { References } & 72\end{array}$ 


\section{Introduction}

High-energy neutrinos, with energies much larger than $100 \mathrm{MeV}$, must be emitted as a by-product of collisions of charged cosmic rays with matter; in fact, only neutrinos provide incontrovertible evidence for hadronic acceleration. Since they can escape much denser celestial environments than light, they can be tracers of processes which stay hidden to traditional astronomy. At the same time, however, their extremely low reaction probability makes their detection extraordinarily difficult.

First ideas to search for cosmic neutrinos other than those from the Sun date back to the late fifties. In 1960, K. Greisen proposed a 3000 ton underground Cherenkov detector to record neutrinos emitted by the Crab nebula [1]. He was flanked by F. Reines [2], who realised, however, that "the cosmic neutrino flux cannot be usefully predicted" - i.e. that a mass of 3000 tons may be far too small. In the same year, M. Markov made his groundbreaking proposal "to install detectors deep in a lake or in the sea to determine the direction of charged particles with the help of Cherenkov radiation" [3]. In the decades since then it was realised that high-energy neutrino astronomy requires detectors of a cubic kilometre or larger that can indeed only be implemented in open media. Actually, the first project of that size, IceCube at the South Pole, has just been completed. Two others, KM3NeT in the Mediterranean Sea and GVD in Lake Baikal, are in their preparatory phases. No doubt, we are entering an exciting era of opportunity.

Already now, neutrino astronomy is reality in the low-energy sector, where the detection of neutrinos from the Sun and the supernova SN 1987A was honoured by the 2002 Nobel Prize for physics. Figure 1 shows a compilation of the spectra of dominant natural and artificial neutrino fluxes. No practicable idea exists on how to measure the neutrinos of the $1.9 \mathrm{~K}$ neutrino counterpart to the cosmic microwave background. At higher energies, neutrinos from the Sun, from SN 1987A, from reactors and from the interior of the Earth have already been detected, as have so-called "atmospheric neutrinos" created in cosmic ray interactions in the Earth's atmosphere. Still awaiting detection are high-energy cosmic neutrinos from extraterrestrial sources such as active galactic nuclei (AGN) or from interactions of ultraenergetic protons with the cosmic microwave background [4]. These cosmic neutrinos will hopefully be detected by neutrino telescopes in the next decade, even though predictions for their fluxes are uncertain by orders of magnitude in many cases.

The development of high-energy neutrino astronomy is reflected in a series of previous reviews spanning the period 1995 to 2009 [5-10]. The neutrino telescopes discussed in this review focus on energies beyond a few $\mathrm{GeV}$. First searches for such neutrinos were made in the 1960s in the Kolar Gold Field mine in India and in the East Rand mine in South Africa (for a review see [7]). In the 1980s, the spectrum of atmospheric muon neutrinos was measured with a detector in the Fréjus tunnel between France and Italy, and a first limit on the diffuse flux of extra-terrestrial $\mathrm{TeV}$ neutrinos was set [11]. Over the following decades, the evolution of underground neutrino detectors culminated in two experiments with an area of about $1000 \mathrm{~m}^{2}$ each (see Sect. 3.5 for a discussion of effective areas): MACRO in the Gran Sasso Underground Laboratory in Italy and Super-Kamiokande in the Japanese Kamioka mine. MACRO collected more than thousand atmospheric neutrinos over six years 


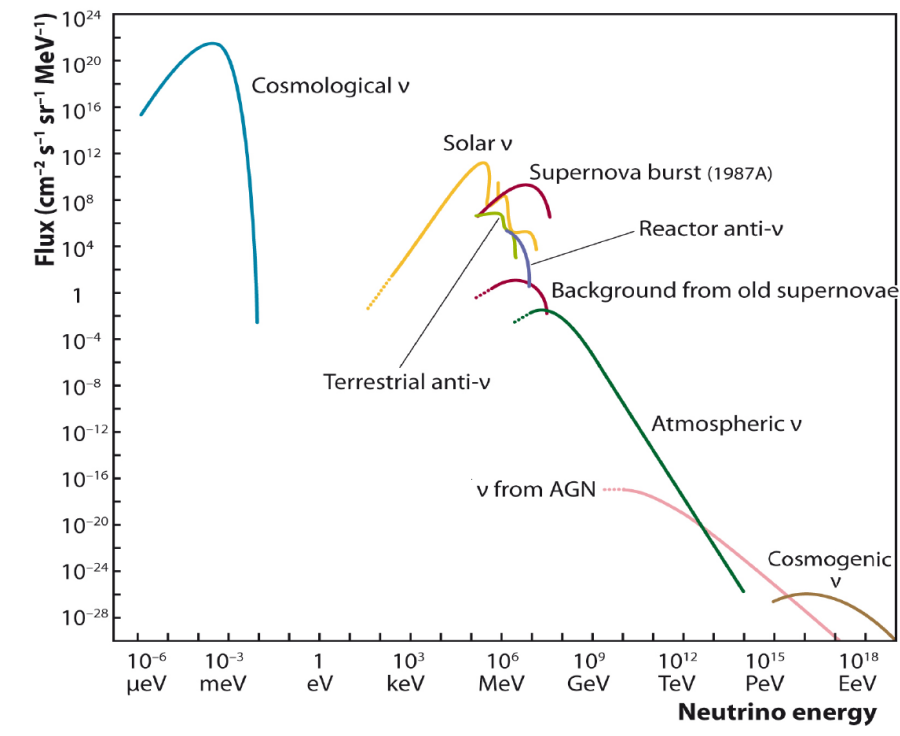

Figure 1. Measured and expected fluxes of natural and reactor neutrinos.

of data taking. Super-Kamiokande, with an even larger data sample, is still in operation. The atmospheric neutrino results from these detectors have demonstrated that neutrinos oscillate between their flavour states $\nu_{\mu}$ and $\nu_{\tau}$, additionally to the $\nu_{e}$ oscillations observed for solar neutrinos [7].

The first-generation detectors in water and ice have beaten the largest underground detectors by a factor of about 30 with respect to their sensitivity to high-energy neutrinos. The second-stage detectors on the cubic-kilometre scale will yield another factor of 30 . Compared to detectors underground we therefore enter a "factor-1000 era". Arguably, this factor is not a guarantee for discoveries. On the other hand it rarely happened in astronomy that improvements of more than an order of magnitude (in sensitivity or in angular or time resolution) came along without discovering new, unexpected phenomena [12. "Nothing is guaranteed, but history is on our side" [13]: In some years we will know whether we indeed have entered an era of discovery or not.

This review is organised as follows: Section 2 summarises the scientific motivation. Apart from the main topic, neutrino astrophysics, it includes the indirect search for dark matter, the study of standard and non-standard neutrino oscillations, the search for exotic particles like magnetic monopoles, super-symmetric Q-balls or nuclearites and - last but not least the investigation of environmental effects, be it in deep natural water or Antarctic ice. The basics of the detection methods are summarised in Sect. 3 . In Sect. 4 the first-generation neutrino telescopes are described, in Sect. 5 the second-generation projects on the cubickilometre scale. A selection of results obtained with NT200 in Lake Baikal, ANTARES in the Mediterranean Sea as well as AMANDA and IceCube at the South Pole is presented in the following Sect. 6. For the highest energies beyond $100 \mathrm{PeV}$, even cubic-kilometre detectors are far too small to detect the feeble neutrino fluxes expected. This is the realm of new technologies which aim, with a correspondingly high detection threshold, to monitor volumes of 100 cubic kilometres and beyond. These methods are described in Sect. 7. The last section finally gives a summary and tries an outlook to forthcoming developments. 


\section{Scientific Background and Motivation}

The primary motivation to build kilometre-scale neutrino detectors is driven by the observation of charged cosmic rays. Since long, neutrinos have been supposed to be a key messenger to identify the sources of cosmic ray acceleration and to provide a deeper understanding of the associated astrophysical objects. In Sect. 2.1, we recall basic information on cosmic rays and gamma rays which motivate (and constrain) the search for energetic neutrinos. We sketch the astrophysical source candidates for neutrinos from hadronic acceleration processes and relate the predicted fluxes to event rates expected in neutrino telescopes on the cubic-kilometre scale.

The identification of the sources of cosmic rays and, more generally, the opening of a new observational window to the Universe, is arguably the most important, but by far not the only purpose of large neutrino telescopes. Neutrino detectors are multi-purpose devices addressing also questions of particle physics and environmental science. Section 2.2 gives an overview on the particle physics issues studied with the help of neutrino telescopes, and Sect. 2.3 sketches the environmental spin-offs.

\subsection{Neutrinos from cosmic accelerators}

High-energy charged cosmic rays have been known to exist for almost a century, but their origin is still a mystery. Since charged particles are deflected in the inter- and extragalactic magnetic fields, their arrival direction at Earth does not reveal their sources, except at the very highest energies. In contrast, neutrinos, produced at the acceleration sites or during cosmic ray propagation, propagate on straight trajectories and point back to their origin.

Several astrophysical object classes have been proposed as potential particle accelerators. In spite of a vast amount of observational data in all electromagnetic wavelength regimes from radio to gamma - it is, however, still unclear, whether the non-thermal processes in these objects are of electronic or hadronic nature. It appears likely that a final answer to this question will require the observation of neutrinos (cf. Sect. 2.1.2).

\subsubsection{Cosmic rays}

Cosmic rays are charged particles - basically hadrons (protons, light and heavy nuclei) with only a tiny admixture of electrons - that impinge on Earth from outer space, with an energy spectrum extending to energies in excess of $10^{20} \mathrm{eV}(100 \mathrm{EeV})$, see Fig. 2 .

The energy spectrum follows a broken power law $E^{-\alpha}$. The region where the decrease with a power index $\alpha=2.7$ steepens to a power index of $\alpha=3.1$ at about $10^{15} \mathrm{eV}(1 \mathrm{PeV})$ is called the "knee". It is assumed that cosmic rays up to (and even beyond) the knee are of Galactic origin. The main accelerator candidates are supernova remnants (SNR): The shock fronts powered by supernova explosions propagate into the interstellar medium, and by repeated scattering processes across the shock front particles can gain energy ("first-order Fermi acceleration" [15-19]).

The spectrum resulting from first-order Fermi acceleration can be shown to follow roughly an $E^{-2}$ spectrum, with a maximum energy

$$
E_{\max } \propto Z \cdot v \cdot B \cdot L
$$




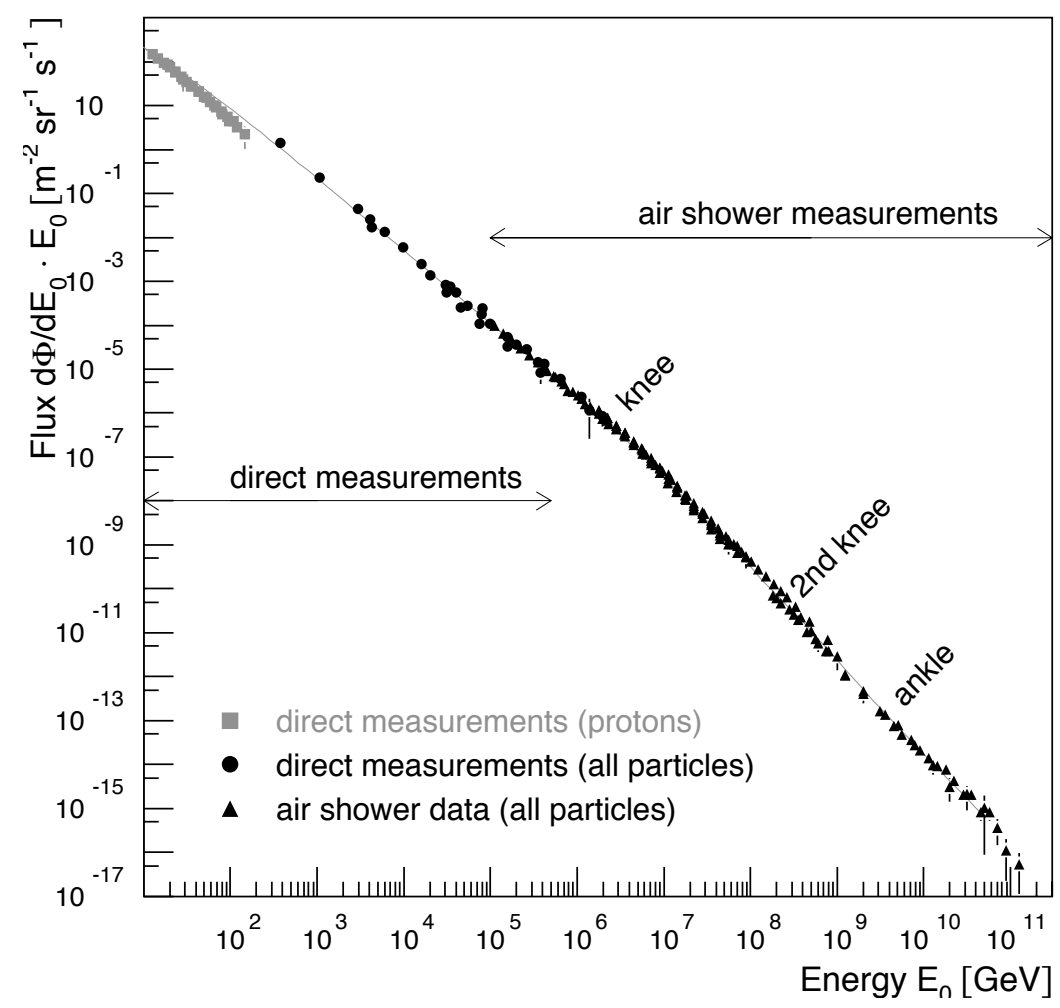

Figure 2. Energy spectrum of cosmic rays, illustrating its main features (knee, ankle, and high-energy cut-off at a few $10^{11} \mathrm{GeV}$ ). Figure taken from [14.

where $Z$ is the charge of the particle, $v$ the velocity of the shock wave, $B$ the magnetic field strength in the acceleration region and $L$ the size of this region.

With eq. (1), the maximum energy to which a SNR can boost particles turns out to be somewhat below the knee, at about $10^{14} \mathrm{eV}$. However, by interactions of cosmic rays with the magnetic fields in the acceleration region, these fields can be amplified, resulting in energies of up to $10^{16} \mathrm{eV}$ (see [20] and references therein). The mechanism of converting kinetic energy of the SNR into energy of accelerated particles is effective over the first $10^{3}-10^{4}$ years of the SNR.

Circumstantial evidence for SNRs as the main sources of cosmic rays can be also derived from the Galactic energy budget. The energy density of Galactic cosmic rays is about $1 \mathrm{eV} / \mathrm{cm}^{3}$ [21], corresponding to $\rho_{\mathrm{CR}}=10^{-12} \mathrm{erg} / \mathrm{cm}^{3}$. The power required to sustain this density is $L=\rho_{\mathrm{CR}} \cdot V / t_{\text {cont }}$, with $t_{\text {cont }} \approx 10^{7}$ years [14] being the average containment time of cosmic rays in the Galaxy and $V \approx 10^{67} \mathrm{~cm}^{3}$ the volume of the visible part of the Galaxy. With $L \approx 10^{41} \mathrm{erg} / \mathrm{s}$, the power to maintain the cosmic ray density turns out to be about $10 \%$ of the power generated by supernovae, which release on average $10^{51}$ erg every 20-50 years, a relation noticed already in 1934 by Baade and Zwicky [22].

Besides supernova remnants, there are further candidates for Galactic particle acceleration, most notably pulsars with their extremely high magnetic fields at the poles, and binary systems with a neutron star or a black hole as one of the partners. The latter often form relativistic radio jets and are then dubbed microquasars (for a review, see [23]).

Somewhere between $10^{17}$ and $10^{18.5} \mathrm{eV}$, at maximum, known Galactic source candidates 
are running out of power and extragalactic sources start dominating the spectrum. Around $10^{18.5} \mathrm{eV}$ the spectrum flattens again to an $E^{-2.7}$ shape, a feature named the "ankle". Almost all cosmic rays with energies above the ankle are assumed to be of extragalactic origin. Even if Galactic sources would accelerate particles beyond the ankle energy, these particles would right away escape the Galaxy since their gyroradius exceeds the size of the Galaxy.

The cut-off of the cosmic ray spectrum at highest energies, confirmed by recent Auger measurements [24], is likely due to interactions with the cosmic microwave background $\left(p+\gamma_{\mathrm{CMB}} \rightarrow \Delta^{+}\right)$and was first predicted by Greisen, Zatsepin and Kuzmin [25, 26]: the "GZK cut-off".

The main candidates for particle acceleration to energies beyond $10^{19} \mathrm{eV}$ are Active Galaxies, Gamma Ray Bursts and starburst galaxies. With eq. (1), the maximum energy to which these objects could accelerate protons is between $10^{20}$ and $10^{21} \mathrm{eV}$. Similarly as for supernova remnants and the energy budget of the Galaxy, there is a suggestive coincidence between the power released by these extragalactic objects and the power required to sustain an $E^{-2}$ flux up to GZK energies. The latter is $10^{-7} \mathrm{eV} / \mathrm{cm}^{3}$, equivalent to $3 \times 10^{-19} \mathrm{erg} / \mathrm{cm}^{3}$ [27. Normalised to the cosmic abundance of AGN and GRB, this density translates to a required power of about $2 \times 10^{44} \mathrm{erg} / \mathrm{s}$ (Active Galaxies) and $3 \times 10^{52} \mathrm{erg} / \mathrm{s}$ (Gamma Ray Bursts) and this indeed is of a similar order as the corresponding electromagnetic energies released by these objects.

For a recent review of cosmic rays we refer to [14]).

\subsubsection{Production of neutrinos}

It has been recognised for half a century [1, 2] that protons from cosmic accelerators would also generate neutrinos, via charged pion production in collisions with the ambient matter or radiation fields, in reactions such as:

$$
\begin{aligned}
p+\text { nucleus } & \rightarrow \pi+X \quad\left(\pi=\pi^{ \pm}, \pi^{0}\right) \\
p+\gamma & \rightarrow \Delta^{+} \rightarrow\left\{\begin{array}{l}
\pi^{0}+p \\
\pi^{+}+n
\end{array}\right.
\end{aligned}
$$

with the subsequent decays $\pi^{0} \rightarrow \gamma \gamma, \pi^{ \pm} \rightarrow \mu^{ \pm} \stackrel{\bar{\nu}}{ }_{\mu}$ and $\mu^{+} \rightarrow e^{+} \bar{\nu}_{\mu} \nu_{e}, \mu^{-} \rightarrow e^{-} \nu_{\mu} \bar{\nu}_{e}$. The resulting neutrino flavour ratio is approximately $\nu_{e}: \nu_{\mu}: \nu_{\tau}=1: 2: 0$ at the sources; neutrino oscillation turns this into a ratio of $\nu_{e}: \nu_{\mu}: \nu_{\tau}=1: 1: 1$ upon arrival at Earth (see Sect. 2.2.3).

The kinematic threshold for process (3) is determined by the photon energies in the radiation field. For ambient photons in the UV region, as characteristic for many stars and accreting objects, it is in the range of several PeV. For the cosmic microwave background, it is at about $10^{19.6} \mathrm{eV}$. If the photon spectrum has a broad spectrum, such as the power-law for photons generated by synchrotron radiation, the threshold is "smeared" to much lower energies. This is particularly important for neutrino astronomy in the $\mathrm{TeV}$ range.

$\mathrm{TeV}$ gamma rays can be produced via the decay of neutral pions, but also by inverse Compton scattering:

$$
e^{-}+\gamma_{\text {low energy }} \rightarrow e^{-}+\gamma_{\text {high energy }}
$$


Actually, most of the measured spectra from $\mathrm{TeV}$ gamma ray sources are compatible with models based on inverse Compton scattering, and in many cases with the so-called synchrotron-self Compton (SSC) model where the photon gas is provided by synchrotron radiation from accelerated electrons (see Fig. 3). Needless to emphasise that pure SSC models are based on electron, not hadron acceleration and do not directly explain the origin of cosmic rays. Pure electron acceleration models are called "leptonic models". In most realistic cases, both electrons and hadrons will be accelerated.

Figure 3 sketches the processes happening in such a combined model. The synchrotron radiation from electrons serves as target for Inverse Compton scattering as well as for proton collisions. Electrons are cooled by synchrotron emission and may boost synchrotron photons to the $10-100 \mathrm{TeV}$ range but certainly not to $\mathrm{PeV}$ energies. The observation of $\mathrm{PeV}$ gamma rays would therefore be a clear proof of hadron acceleration. Unfortunately, the range of $\mathrm{PeV}$ photons does not exceed the size of our Galaxy, since they are absorbed by the process $\gamma_{\mathrm{PeV}}+\gamma_{\mathrm{CMB}} \rightarrow e^{+} e^{-}$.

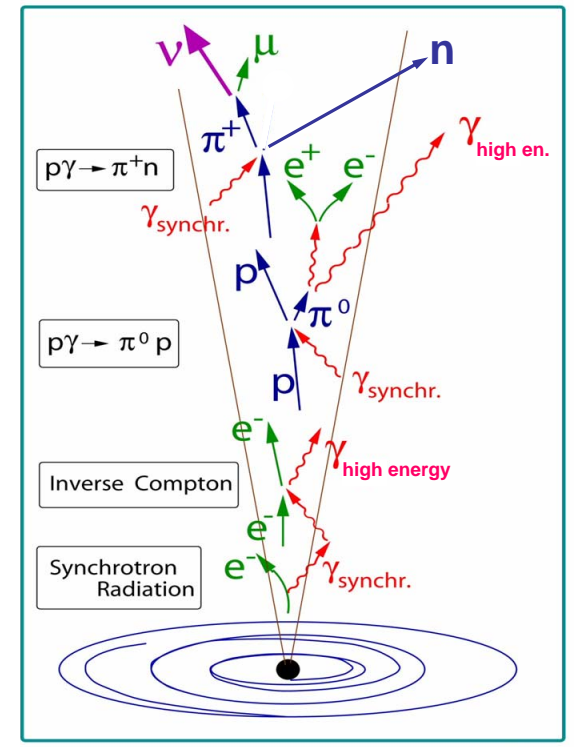

Figure 3. Gamma ray and neutrino production in a jet emitted from an Active Galactic Nucleus, with both hadrons and electrons being accelerated along the jet.

If charged particles are confined by large magnetic fields, only neutral particles can escape the acceleration region. Apart from gamma rays and neutrinos, this can be also neutrons (see top right in Fig. 3), provided the source is sufficiently dilute. Neutrons decaying outside the source would then yield those cosmic ray protons which are detected on Earth and which can be used to constrain the flux of neutrinos (see Sect. 2.1.4).

\subsubsection{Galactic sources}

Until the mid-1990s, only one supernova remnant, the Crab nebula, had been detected in $\mathrm{TeV}$ gamma rays. Therefore, predictions for neutrinos from these sources were not yet on a firm ground at that time. The Crab belongs to a special class of SNR, the pulsar wind nebulae, where a central pulsar emits material into the nebula. The most likely sources of Galactic cosmic rays are shell-type supernova where this effect is absent or not essential. 
In the mean time the situation has changed dramatically. Imaging Air Cherenkov Telescopes like H.E.S.S., MAGIC and VERITAS have detected more than hundred sources of $\mathrm{TeV}$ gamma rays, amongst them about $30 \mathrm{SNRs}$ (for recent reviews see [28, 29]). From the observed fluxes of gamma rays, estimates or upper bounds on the flux of neutrinos can be derived, assuming that most of the observed gamma rays stem from decays of $\pi^{0}$ s generated according to eqs. (2) and (3). Keeping in mind that high-energy gamma rays may well emerge from inverse Compton scattering, eq. (4), their observation is not a proof that the source accelerates hadrons rather than only electrons. A certain test can be provided by detailed information on the $\mathrm{MeV}-\mathrm{GeV}$ part of the gamma spectrum and by information on its high-energy cut-off. For Galactic sources, the morphology of gamma ray emission can be studied and provides additional information. In this context, we note the observation of the supernova remnant RX J1713.7-3946 with the H.E.S.S. telescope [30]. The image of this source (see Fig. 4, left) shows an increase of the gamma flux from the direction of known molecular clouds. The effect can be attributed to protons accelerated in the SNR and then interacting with the clouds. The spectrum of gamma rays above some $100 \mathrm{GeV}$ is well compatible with expectations for the decay of $\pi^{0} \mathrm{~s}$ from proton interactions. Recent measurements with the Fermi-LAT instrument [31, however, indicate that the spectrum at lower energies may be better described by leptonic models. This is in accordance with $\mathrm{X}$-ray line emission around $1 \mathrm{keV}$ which should be produced along with pion decays but has not been observed with the Suzaku satellite detector [32]. Unambiguous Galactic sites of hadronic acceleration have thus yet to be identified. Extended gamma ray sources which trace the density of molecular clouds, such as the SNR Vela (RX J0852.0-4622) [33] and a large region near the Galactic Centre [34], remain good candidates.
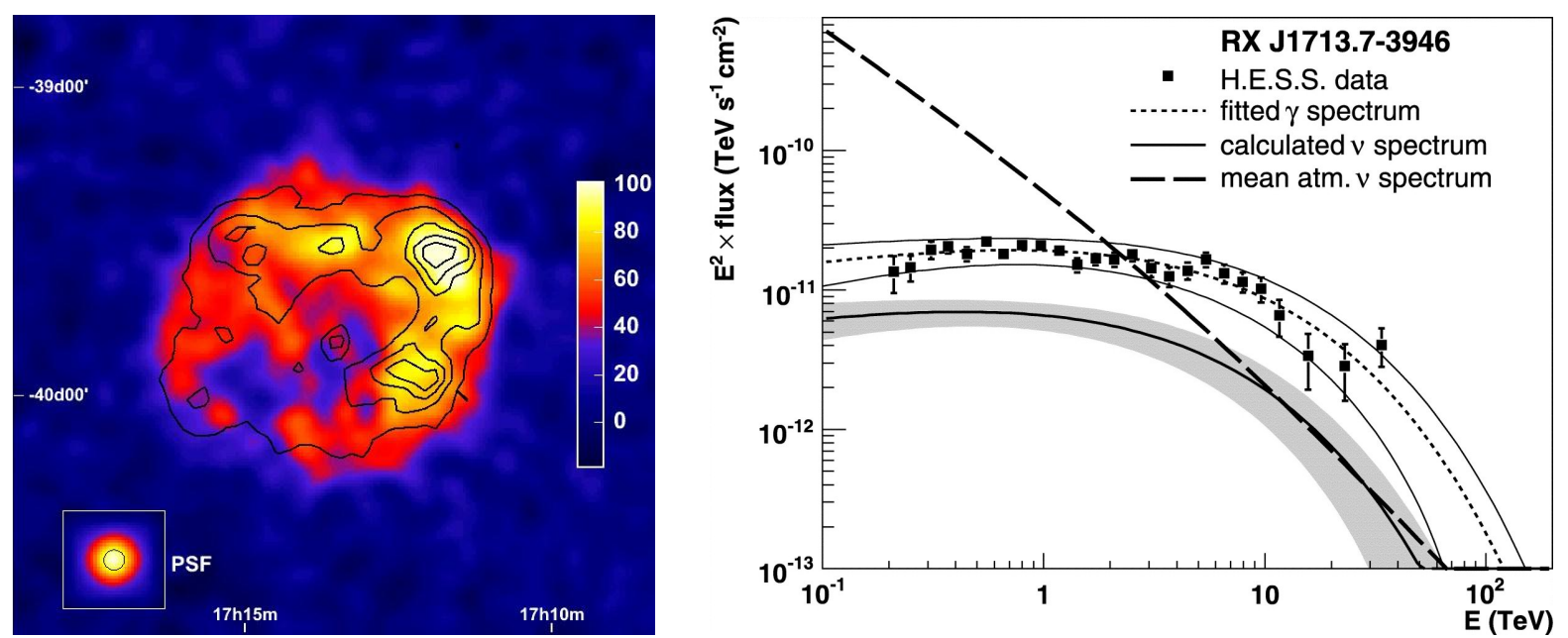

Figure 4. Left: Gamma ray image of the supernova remnant RX J1713.7-3946, recorded with the H.E.S.S. telescope; superimposed are contours of X-ray surface brightness recorded by the ASCA satellite (figure taken from [35]). Right: Measured gamma flux from RX J1713.7-3946 and the related neutrino flux estimated under the assumption that the gamma emission is of purely hadronic origin (figure taken from [36]). 
In [36, the expected neutrino flux from the SNR RX J1713.7-3946 is calculated (cf. Fig 4 , right). Based on the assumption that all observed gamma rays stem from $\pi^{0}$ decays, the authors calculate the neutrino flux expected from $\pi^{ \pm}$decays. For five years of data taking with a cubic-kilometre detector and a threshold at $1 \mathrm{TeV}$, the number of events is calculated to be between 7 and 14, over a background of 21 atmospheric neutrino events in a $2.1^{\circ}$ search cone (resulting from the $1.3^{\circ}$ diameter of the source, multiplied with a factor of 1.6 to achieve optimal sensitivity). A threshold of $5 \mathrm{TeV}$ results in $2.6-6.7$ signal events and 8.2 atmospheric neutrino events. Cutting at higher energies will eliminate not only the atmospheric background but also the signal, cutting at lower energies will significantly worsen the signal-to-background ratio. These estimates suggest that the positive effect of a detector threshold much below $1 \mathrm{TeV}$ will be small, at least for steady sources. It is also clear that neutrinos from this particular source will be hardly observable if the gamma flux of hadronic origin is sub-dominant; in this case, however, the quest of identifying the sites of hadronic acceleration starts afresh, and neutrino observations will be more crucial than ever in solving this puzzle.

Several candidates for sources with hadron acceleration beyond the knee ("Pevatrons") have been identified in the Cygnus region by the Milagro collaboration [37, 38]. The gamma ray spectrum of the strongest of these sources, MGRO J1908+06, is consistent with an $E^{-2}$ behaviour between $500 \mathrm{GeV}$ and $40 \mathrm{TeV}$. It does not show evidence for a cut-off, in accordance with the expectations for Pevatrons, which should emit gamma rays with energies up to several hundred $\mathrm{TeV}$. In [39], the associated neutrino fluxes from the six identified Milagro source candidates have been calculated and the event rates in the IceCube neutrino telescope estimated. In a simulated neutrino sky-map for 5 years of data taking, two of the sources are discernible "by eye" when applying a lower energy threshold of $40 \mathrm{TeV}$. Stacking all six sources, an excess is found with a Poisson probability for being a background fluctuation of smaller than $10^{-3}$ for lower energy thresholds anywhere between 10 and $100 \mathrm{TeV}$. The simulation assumes a gamma-ray cut-off at $300 \mathrm{TeV}$.

Similarly close to the sensitivity of cubic-kilometre telescopes are the expected neutrino fluxes from microquasars, as for instance LS I +61303 and LS 5039, where the latter could provide a handful of neutrino events per year [40].

Practically all publications of the last years (see references above and e.g. [36, 41-45]) come to the unanimous conclusion that cubic-kilometre detectors will just "scrape" the detection region. The present estimates suggest that the sensitivity of a cubic-kilometre telescope is "tantalisingly (and frustratingly) close" [41] to the expectations for the brightest observed Galactic TeV gamma sources.

\subsubsection{Extragalactic sources}

There is much less observational guidance to predict neutrino fluxes from extragalactic than for Galactic sources. The predictions for individual sources have order-of-magnitude character. The best-motivated extragalactic candidates for high energy neutrino emission are Active Galactic Nuclei (AGN), Gamma Ray Bursts (GRB) and galaxy clusters.

- Active Galactic Nuclei host a super-massive black hole with $10^{6}-10^{9}$ solar masses in their extremely bright centre. The black hole accretes matter and thus transforms huge 
amounts of gravitational energy into radiation, typically of the order of $10^{44} \mathrm{erg} / \mathrm{s}$. This energy can also be converted to kinetic energy of accelerated particles, see also Fig. 3. Blazars, a particular class of AGN where the jet is aligned closely to the line of sight, turned out to be strong gamma emitters, with 119 sources at $\mathrm{GeV}$ energies listed in the 2009 Fermi bright gamma source list [46] and 18 at TeV energies being reported in [47. Gamma ray emission from blazars is often highly variable, with the most extreme variation observed by H.E.S.S. for the blazar PKS 2155-304: An increase by two orders of magnitude within one hour [48. Naturally, the observation of neutrino events from such a source and within such a short time window would be rather significant. Another interesting outburst has been observed from the blazar 1ES 1959+650 [49]. This was an "orphan flare", where the $\mathrm{TeV}$ emission was not accompanied by X-ray emission, as it typically would be for SSC models. A hadronic model does not require such a correlation between $\mathrm{TeV}$ and X-ray (synchrotron) emission; therefore orphan flares are interesting environments to search for neutrino emission.

- Gamma Ray Bursts are the most cataclysmic phenomena in the Universe, releasing huge amounts of energy in gamma rays within milliseconds to minutes. The favoured explanation for the longer bursts is the collapse of a massive star into a black hole. The so-called fireball model of GRB assumes that a central engine ejects large amounts of mass within a short time interval which form successive plasma shells and have typical Lorentz factors of $\Gamma=100-1000$. When the outer shells slow down they are hit by the inner shells and internal shock fronts are piling up. Along these fronts, electrons and protons are accelerated. Electrons are cooled by synchrotron radiation, protons can be accelerated up to energies as high as $10^{21} \mathrm{eV} \mathrm{[50].} \mathrm{When} \mathrm{the} \mathrm{shells} \mathrm{run} \mathrm{into} \mathrm{the}$ interstellar medium, external shocks are built up, leading to afterglow emission visible in X-ray, optical and radio wavelengths. Neutrino emission has been calculated for three phases: the precursor phase when the jet is still forming and no electromagnetic radiation is escaping [51]; the "prompt" phase coinciding with the burst in gamma rays (see e.g. [50]); and the afterglow phase [52]. We will return to these predictions when presenting experimental bounds in Sect 6 .

- Starburst galaxies are galaxies undergoing an episode of large-scale star formation, where the central regions eject a galactic-scale wind driven by the collective effect of supernova explosions and winds from massive stars. Recently, the starburst galaxies NGC253 (southern hemisphere) and M82 (northern hemisphere) have been detected by the H.E.S.S. and VERITAS telescopes [53, 54]. The gamma ray flux at several hundred $\mathrm{GeV}$ suggests cosmic ray densities of two to three orders of magnitude above that in our own Galaxy. Following [55], the cumulative neutrino flux of all starburst galaxies is detectable with cubic-kilometre detectors.

Predictions for the integrated flux from all extragalactic sources are based on the observed gamma and X-rays fluxes or those of charged cosmic rays above $10^{18} \mathrm{eV}$. A selection of predictions and bounds is shown in Fig. 5. Early normalisations as e.g. in [56] assumed neutrinos would be produced in the cores of AGN, accompanied by X-ray emission, and 
that most of the observed X-ray background was non-thermal radiation from a superposition of the fluxes from unresolved AGN. This model as well as others violated upper bounds derived from the observed cosmic ray spectrum. Subsequent observations of these AGN showed that most of the X-ray emission is thermal and therefore cannot be directly related to the production of relativistic particles. Relying on $\mathrm{MeV}$ gamma ray rather than X-ray observations, the authors scaled down the original prediction by an order of magnitude ([57], cf. curve 2 in Fig. 5).

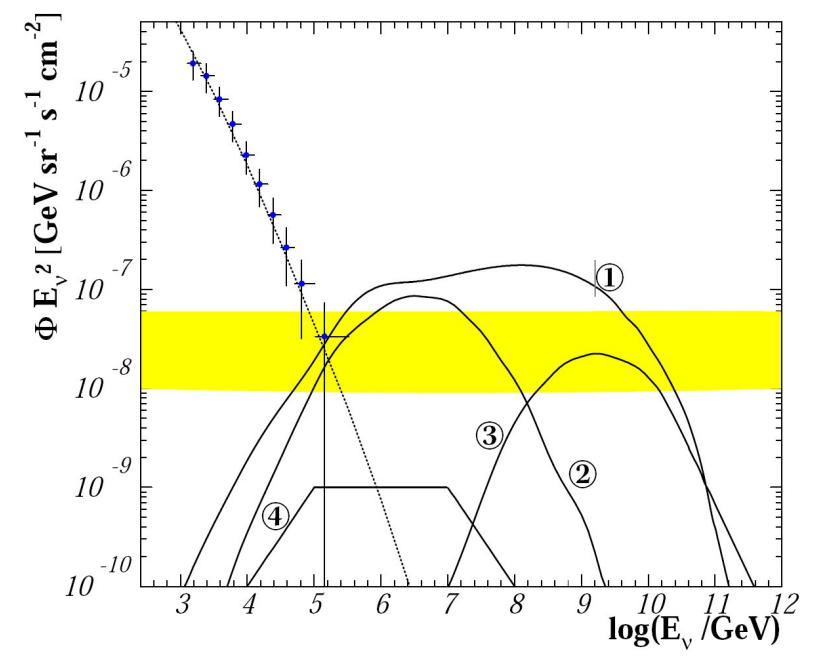

Figure 5. Spectra of diffuse neutrino fluxes, multiplied with $E^{2}$. Points: AMANDA measurements of the atmospheric neutrino flux [58], compared to the prediction from [59] (dotted line). Horizontal band: $E^{-2}$ upper bound derived from charged cosmic rays at $E>10^{19} \mathrm{eV}$ [60], with the width reflecting the uncertainty of cosmic evolution parameters. Numbered curves: predictions for the diffuse flux of neutrinos from all AGN $(1,2)$ [57, 61, of cosmogenic neutrinos (3) [4] and of neutrinos from GRBs, assuming that they are the sources of highest energy charged cosmic rays (4) [50]. Figure courtesy of J. Becker.

Two upper bounds on the diffuse neutrino flux, both derived from charged cosmic ray fluxes, are frequently used as benchmarks. The first ("Waxman-Bahcall bound" [60]) is normalised to the cosmic ray flux at about $10^{19} \mathrm{eV}$. Assuming a generic $E^{-2}$ spectrum for all extragalactic sources, the authors obtain a limit of $E^{2} \Phi_{\nu}=1-5 \times 10^{-8} \mathrm{GeV} \mathrm{cm}^{-2} \mathrm{~s}^{-1} \mathrm{sr}^{-1}$, where $\Phi_{\nu}$ is the neutrino flux differential in energy, time, area and solid angle and the uncertainty is given by different cosmic evolution models (coloured band in Fig. 5). This estimate assumes that the sources are sufficiently dilute, so that neutrons can escape, decay and provide the observed cosmic rays to which the estimate is normalised. If the sources are opaque even for neutrons, the only remaining estimator is electromagnetic radiation. The decay of $\pi^{0} \mathrm{~s}$ (co-produced with charged pions producing neutrinos) yields gamma rays which develop electromagnetic cascades. The energy of gammas escaping the source is mostly in the range $1 \mathrm{MeV}$ to $100 \mathrm{GeV}$. The diffuse gamma-ray background above $30 \mathrm{MeV}$ was measured by the EGRET satellite [62] to be $E^{2} \Phi_{\gamma}=1.37 \times 10^{-6} \mathrm{GeV} \mathrm{cm}^{-2} \mathrm{~s}^{-1} \mathrm{sr}^{-1}$ and sets a bound of similar size to the neutrino flux (not shown in the figure), which hardly can be circumvented by more sophisticated assumptions on the character of the sources. Recent Fermi data [63] suggest [64] that this "gamma bound" (or "cascade bound", originally derived in [65]) is close to the Waxman-Bahcall bound, and actually has already been superseded by experimental upper limits from the running underwater/ice telescopes (see below). In a cubic-kilometre detector, the Waxman-Bahcall flux would lead to 100-500 events per year.

Contrary to Waxman and Bahcall, Mannheim, Protheroe and Rachen (MPR) [61] assume that a significant part of the observed cosmic ray spectrum between $10^{16} \mathrm{eV}$ and 
$10^{19} \mathrm{eV}$ is due to extragalactic rather than Galactic sources. Interpreting this spectrum as a superposition of spectra from many extragalactic source classes, each with a different cut-off, the neutrino bound considerably weakens towards lower energies and is about $E^{2} \cdot \mathrm{d} N / \mathrm{d} E \approx 5 \times 10^{-7} \mathrm{GeV} \mathrm{cm}^{-2} \mathrm{~s}^{-1} \mathrm{sr}^{-1}$, at a few $10^{14} \mathrm{eV}$. Meanwhile, also this bound is only of historical interest, due to the new results from the Fermi satellite and from neutrino telescopes.

A non-astrophysical contribution to the diffuse neutrino background is assumed in "topdown scenarios", where cosmic rays of highest energy are due to cascade decays of long-lived super-heavy particles at unification-scale energies $\left(10^{24}-10^{25} \mathrm{eV}\right)$. Such processes inevitably produce high-energy neutrinos and photons. An estimate of the resulting neutrino event rate in IceCube of up to 40 events per year [66] has meanwhile been superseded by recent, tight limits from Auger on the photon contribution to energetic cosmic rays [67].

The most exciting discovery of neutrino astronomy would be the detection of point sources rather than just a high-energy excess in diffuse fluxes. However, experimental limits for diffuse fluxes are setting bounds for expected point-source fluxes. The argument is as follows: Contributions to the diffuse flux will come from all the observable universe, up to a distance $c / H_{0}$, whereas point sources will be visible at a level of several events per source only up to a limited distance of a few hundred Mpc, assuming reasonable maximum luminosities per source. For a homogeneous distribution of extragalactic sources, one therefore can derive a limit on the number of observable point sources. In [41 the following assumptions are made: a homogeneous source density in a Euclidean universe; a "typical" (and similar) source luminosity $L_{\text {source }}$ for all sources; an $E_{\nu}^{-2}$ spectrum of the neutrino fluxes. Given an experimental limit $K_{\text {diffuse }}$ on the diffuse neutrino flux and a sensitivity $C_{\text {point }}$ to point sources, the expected number of resolvable extragalactic point sources, $N_{s}$ scales as

$$
N_{s} \propto \frac{K_{\text {diffuse }} \cdot \sqrt{L_{\text {source }}}}{C_{\text {point }}^{3 / 2}} .
$$

With the present diffuse flux limit from IceCube and its point source sensitivity, one obtains $N_{s} \approx 0.2-2$ (in analogy to an estimate made in [68] for the AMANDA diffuse limit). This means that, under the given assumptions, a cubic-kilometre detector would have a remaining chance to detect extragalactic point sources. Note, however, that a few individual, very close sources could circumvent the homogeneity assumption and be well observable. Also, point sources with cut-offs below a few hundred TeV would not be covered by the argument above since, in order to obtain the best sensitivity for diffuse fluxes, a lower energy cut at about $100 \mathrm{TeV}$ has to be used [69]. In particular this implies that the argument of [41] does not apply to Galactic sources since they are not homogeneously distributed and typically are expected to have cut-off energies below $100 \mathrm{TeV}$.

\subsection{Particle physics and exotic phenomena}

Beyond charting the high energy universe, neutrino telescopes may reveal first signatures of new physics beyond the standard model. Opportunities include the indirect search for dark matter particle candidates, the search for super-heavy exotic particles like supersymmetric Q-balls or magnetic monopoles, or the search for deviations from the established 
neutrino oscillations which may result from violation of Lorentz invariance. We sketch these opportunities in the following.

\subsubsection{Indirect search for dark matter}

Favoured dark matter candidates are Weakly Interacting Massive Particles (WIMPs). They are preferentially discussed in the framework of the minimal supersymmetric standard model (MSSM), see [70] and references therein. Specifically, the lightest supersymmetric particle is considered a good WIMP candidate, with predicted masses in the range from a few $\mathrm{GeV}$ to a few tens of $\mathrm{TeV}$ [71]. A more constrained class of theories is based on minimal supergravity (mSUGRA); see [72] for a comparison of MSSM and mSUGRA. WIMPs can be trapped in the gravitational potential of celestial bodies such as the Earth or the Sun through elastic scattering and would accumulate in the cores of these objects. If WIMPs are Majorana particles (as in supersymmetry), they may subsequently annihilate once sufficient densities are reached. Neutrinos are produced via decay of the annihilation products [73]. The mean neutrino energy depends on the WIMP mass and the annihilation final state. For instance, quark-antiquark states result in softer, $W^{+} W^{-}$states in harder neutrino spectra.

The capture rate depends on the mass of the celestial body and on local density, mean velocity and scattering cross section of the WIMPs on nuclei. Slower WIMPs are captured more efficiently than faster WIMPs. The scattering cross section can be spin-dependent or spin-independent; the capture rate thus depends on the spin composition of the nuclei in the celestial body (capture in the Sun, e.g., is dominated by scattering on hydrogen nuclei with spin $1 / 2$ and is therefore particularly effective for a spin-dependent interaction). The scattering cross section also depends on the mass ratio of the WIMP and the scattered nucleus. For instance, the Sun with its lighter nuclei captures light WIMPs more efficiently, the Earth gains with respect to heavier WIMPs. The annihilation rate scales with the square of the accumulated WIMP density (which likely has reached equilibrium for the Sun but less likely for the Earth) and with the annihilation cross section (which is related to the scattering cross section through the underlying theoretical model).

WIMP detection via secondary particles from annihilations (in particular gammas or neutrinos) is called "indirect detection", in contrast to "direct detection" of recoil nuclei from elastic WIMP-nucleus scattering. Results from both methods can be related (see Sect. 6), but one has to keep in mind that direct event rates are highest for fast WIMPs and scale with the WIMP density, whereas indirect rates are highest for slow WIMPs and scale with the square of the WIMP density. In this respect, the two methods are complementary. Whereas direct and indirect searches are on similar footing with respect to spin-independent scattering, indirect searches win when it comes to neutralino capture via spin-dependent scattering in the Sun [74]. Consequently, limits on the spin-dependent scattering cross section from indirect searches are by nearly two orders of magnitude better than those from direct searches. For detailed reviews on dark matter searches, we refer to [74 76].

An alternative and in many ways orthogonal approach is the search for WIMP candidates at accelerators, in particular at the LHC [77. The forthcoming years will be particularly interesting in this respect. One has to keep in mind, however, that a discovery at LHC would not yet pin down the dark matter properties since it would remain to be shown that 
the LHC particles actually (exclusively) form the astrophysical dark matter. In that sense, a full picture of the very nature of dark matter (if it were supersymmetric particles) will be obtained only by combining results from all three methods [75].

\subsubsection{Super-heavy particles}

Neutrino telescopes can be used to search for several exotic objects on the mass scale much beyond that of WIMPs which may lead to characteristic signal patterns. Here, we mention the three most popular ones: magnetic monopoles, supersymmetric Q-balls and nuclearites. The predicted mass of magnetic monopoles ranges between $10^{4}$ and $10^{19} \mathrm{GeV}$, nuclearites could have masses from a few hundred $\mathrm{GeV}$ up to the mass scale of neutron stars, and Q-balls up to $10^{27} \mathrm{GeV}$. The expected signals for these particles are similar; a clear assignment is therefore excluded for a single event, but may be possible if angular and light yield distributions can be studied for a sample of several events.

- Magnetic monopoles: Magnetic monopoles have been introduced by P. Dirac in 1931 in order to explain the quantisation of electric charge [78]. After decades of unsuccessful monopole searches at accelerators and in cosmic rays, the efforts gained new momentum when monopoles turned out to be a consequence of most variants of Grand Unified Theories [79]. A phase transition in the early Universe at $10^{-34} \mathrm{~s}$ might have filled the Universe with a significant amount of monopoles.

Measurements and estimates of cosmic magnetic fields suggest that they could accelerate magnetic monopoles lighter than $10^{14} \mathrm{eV}$ (sub-GUT-scale) to relativistic velocities [80, 81]. The magnetic charge of monopoles obeys the Dirac quantisation rule $g=n \cdot e /(2 \alpha)$, with $n=1,2,3, \ldots$ and $\alpha=1 / 137$ ( $e$ being the elementary charge). A magnetic monopole with unit charge $g=137 e / 2$ and a velocity above the Cherenkov threshold in water or ice $(\beta>0.75)$ would emit Cherenkov light along its path. The light intensity would exceed that of a minimally ionising muon by a factor of 8300 , this providing a rather unique signature in a neutrino telescope.

Typical GUT versions predict monopoles with masses of $10^{16} \mathrm{GeV}$ and more. These monopoles would have typical virial velocities peaking at $\beta=10^{-4}-10^{-3}$. They might catalyse baryon decays along their path which would be detected via the decay particles 82. For certain regions of the parameter space spanned by catalysis cross section and monopole velocity, the Cherenkov light from the secondary particles would create a pattern of a slowly propagating light source on a straight trajectory, well observable in a neutrino telescope.

- Nuclearites: Nuclearites ("strange quark matter" or "strangelets") are hypothetical aggregates of $u, d$ and $s$ quarks combined with electrons, to adjust electric neutrality. They might be stable for baryon numbers ranging from those of usual nuclei up to those of neutron stars $\left(A \approx 10^{57}\right)[83$, 84. Nuclearites could have been produced in the primordial Universe or in certain astrophysical processes like the collision of neutron stars. They would induce a thermal shock wave along their path through the detector medium. For virial velocities, the corresponding Planck radiation could reach 
$10^{3} \mathrm{~K}$. A light source so slow and bright would produce a very specific time pattern similar to that of a slow GUT monopole.

- Supersymmetric Q-balls: Q-Balls are hypothesised coherent states of squarks, sleptons and Higgs-fields [85]. These SUSY soliton states could be stable for masses $M \gg$ $10^{15} \mathrm{GeV}$, the life time for smaller masses is unknown. Stable Q-Balls could provide a relevant contribution to Dark Matter. Decays of unstable Q-balls could produce WIMPs, with properties not compatible with the conventional thermal origin [84]. The discovery of Q-Balls would have an enormous impact on the understanding of matter-antimatter symmetry emerging in the early Universe [86 88].

Neutral Q-ball objects (SENS, supersymmetric Electrically Neutral Solitons) could catalyse proton decays along their path, similar to GUT monopoles. Electrically charged Q-ball objects (SECS, supersymmetric electrically charged solitons) would produce light in a similar way as nuclearites.

\subsubsection{Neutrino oscillations}

We meanwhile know that neutrinos change their flavours during propagation. There are two standard mechanisms that contribute to this phenomenon, a mixing between mass and flavour eigenstates (inducing "vacuum oscillations"), and flavour-dependent forward scattering amplitudes in matter ("MSW effect" [89, 90]). Non-standard oscillation effects could e.g. be associated to a violation of Lorentz invariance. Neutrino telescopes have the potential to study the high-energy regime of neutrino oscillations.

\section{Standard oscillations:}

Neutrino data from solar, atmospheric, reactor and accelerator experiments support the concept of neutrino oscillations. The weak flavour eigenstates $\nu_{e}, \nu_{\mu}, \nu_{\tau}$ are linear combinations of mass eigenstates $\nu_{1}, \nu_{2}, \nu_{3}$. For the simplified case of two flavours $\nu_{\mu}, \nu_{\tau}$ and two mass eigenstates $\nu_{2}, \nu_{3}$ one has:

$$
\begin{aligned}
& \nu_{\mu}=\nu_{2} \cos \theta_{23}+\nu_{3} \sin \theta_{23} \\
& \nu_{\tau}=-\nu_{2} \sin \theta_{23}+\nu_{3} \cos \theta_{23} .
\end{aligned}
$$

If the masses $m_{2}$ and $m_{3}$ are different, quantum mechanical time evolution of an initial $\nu_{\mu}$ state induces a non-zero transition probability to $\nu_{\tau}$. The survival probability for the muon neutrino is

$$
P\left(\nu_{\mu} \rightarrow \nu_{\mu}\right)=1-\sin ^{2}\left(2 \theta_{23}\right) \cdot \sin ^{2}\left(\frac{1.27 \Delta m_{23}^{2} \cdot L}{E_{\nu}}\right),
$$

where $L$ (in $\mathrm{km}$ ) is the distance travelled by the neutrino, $E_{\nu}$ (in $\mathrm{GeV}$ ) its energy and $\Delta m_{32}^{2}=m_{3}^{2}-m_{2}^{2}$ (in $\mathrm{eV}^{2}$ ). The three-flavour case is governed by two independent differences of mass squares and three mixing angles. The best-fit oscillation parameters derived from present data are [91]:

$$
\begin{array}{rlrl}
\left|\Delta m_{31}^{2}\right| & =2.40 \times 10^{-3} \mathrm{eV}^{2} \approx\left|\Delta m_{23}^{2}\right| & \sin ^{2} 2 \theta_{23} & \simeq 1 \\
\Delta m_{21}^{2} & =7.65 \times 10^{-5} \mathrm{eV}^{2} & \sin ^{2} \theta_{12} & =0.304 \\
& \sin ^{2} \theta_{13} & <0.056 \quad(3 \sigma) .
\end{array}
$$


If an atmospheric muon neutrino crosses the Earth along its full diameter, the first minimum of the survival probability occurs around $24 \mathrm{GeV}$ (see Fig. 6), the second at $8 \mathrm{GeV}$. For shorter propagation paths through the Earth, the minima occur at correspondingly reduced energies and become unobservable when approaching the horizon.

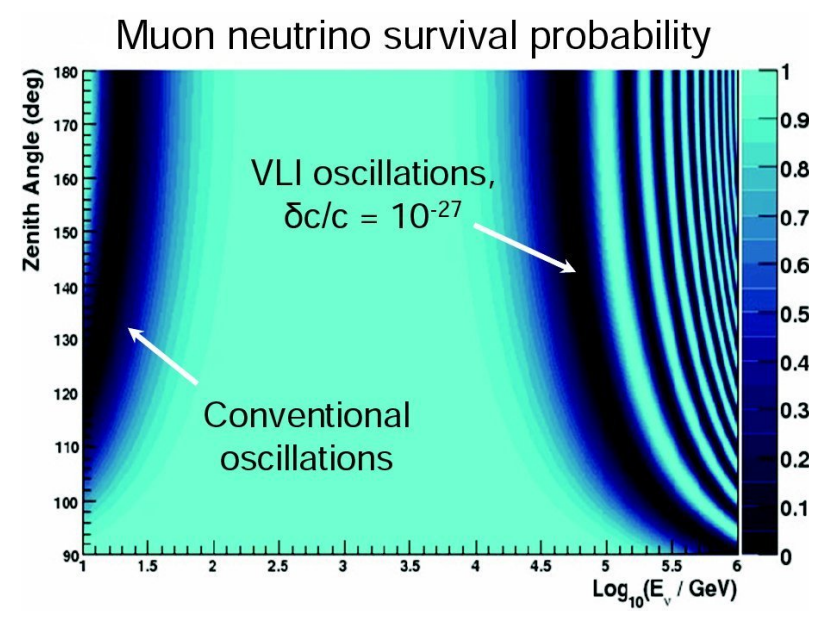

Figure 6. The probability of muon neutrino disappearance as a function of neutrino energy $E_{\nu}$ and zenith angle for atmospheric neutrinos A zenith of $180^{\circ}$ refers to a vertically-upward going neutrino, i.e. propagation along the full Earth diameter. A zenith angle of $90^{\circ}$ marks horizontal propagation with a baseline of a few $100 \mathrm{~km}$. The pattern at lower energies corresponds to "standard oscillations" (without taking into account matter effects), the patterns at higher energies would be expected for non-standard oscillations due to the violation of Lorentz invariance (see text).

If their energy threshold is as low as about $10 \mathrm{GeV}$ (like for IceCube's DeepCore, see Sect. 5), underwater/ice detectors can approach the range of conventional oscillations at high energies [92, complementary to underground detectors like Super-Kamiokande which are mostly sensitive to lower energies.

For Galactic or extragalactic distance scales, kpc to Mpc and beyond, only average oscillation probabilities are observable since even tiny energy differences cause large oscillation phase shifts; in addition, the emission regions may well be larger than oscillation wavelengths. It can be shown that the mixing parameters listed in eq. (8) transform a produced flavour ratio of $\nu_{e}: \nu_{\mu}: \nu_{\tau}=1: 2: 0$ to an observed one of $\nu_{e}: \nu_{\mu}: \nu_{\tau}=1: 1: 1$ [93, 94].

\section{Matter effects and neutrino mass hierarchy:}

One of the remaining puzzles of neutrino physics is the hierarchy of the mass eigenstates $\nu_{1}, \nu_{2}, \nu_{3}$. We know that $m_{2}$ is larger than $m_{1}$, but we do not know whether $m_{3}$ is the largest (normal hierarchy) or the smallest mass (inverted hierarchy). It is expected that eventually double-beta decay experiments may provide the answer (in case neutrinos are Majorana particles). Equally promising are long-baseline neutrino accelerator experiments, provided $\sin ^{2} 2 \theta_{13} \gtrsim 0.001$. Also a 100 Megaton detector for neutrinos may give the answer if $\sin ^{2} 2 \theta_{13}$ is not too much below 0.1 and the detection threshold is $5-10 \mathrm{GeV}$ [92, 95, 96]. This is the energy range where matter-induced oscillations become important.

For neutrinos below $15 \mathrm{GeV}$ passing through the centre of the Earth, the MSW effect slightly enhances the $\nu_{\mu}$ oscillation probability and suppresses the $\bar{\nu}_{\mu}$ oscillation probability. For the inverted hierarchy, in contrast, the oscillation probability for $\bar{\nu}_{\mu}$ is enhanced and for $\nu_{\mu}$ suppressed. Water/ice neutrino telescopes cannot distinguish between neutrinos and antineutrinos (i.e. identify the charges of the secondary muons). However, in the relevant energy range the interaction cross sections of neutrinos and anti-neutrinos differ by about 
a factor of two: $\sigma(\nu) \approx 2 \sigma(\bar{\nu})$. This translates into a difference in the number of observed muon events (about $5 \%$ for $\sin ^{2} 2 \theta_{13}=0.1$ ). Based on the statistical discrimination with respect to the number of events below $15 \mathrm{GeV}$, it may therefore be possible to distinguish normal from inverted hierarchy with DeepCore. Needless to say that firmly establishing such a small difference requires excellent knowledge of the detector systematics. Given the complicated optical properties of ice, it remains to be demonstrated whether systematic uncertainties for these low energies can be kept sufficiently small. In this respect, a densely instrumented water detector may be superior.

Non-standard oscillations:

Many quantum gravity (QG) models suggest that Lorentz invariance may be violated and that this effect may be seen in neutrino oscillations [97-99]. Conventional oscillations are due to different mass eigenstates. Violation of Lorentz invariance (VLI) may occur if in addition there are also different velocity eigenstates, each with its own limiting velocity, differing from the speed of light. The VLI magnitude depends on the velocity splitting $\Delta c / c=\left(c_{1}-c_{2}\right) / c$. Assuming maximum conventional and VLI mixing and the same phase, the muon survival probability can be written as

$$
\left.P_{\nu_{\mu} \rightarrow \nu_{\mu}}\right|_{\text {max. mixing }}=1-\sin ^{2}\left(\frac{\Delta m^{2} L}{4 E}+\frac{\Delta c}{c} \frac{L E}{2}\right),
$$

where the first term comes from standard oscillations (see eq. (7)) and the second from VLI mixing. Note that the two effects scale differently with energy, the one with $1 / E$, the other with $E$ or, more generally, with $E^{n}$ if $\Delta c / c$ is replaced by a generalised VLI term $\Delta \delta=\Delta c / c \times E^{1-n}$.

Another possible consequence of QG is the evolution of pure neutrino states to mixed states via interaction with the foamy space-time itself (quantum decoherence). The resulting effect can be characterised by a set of parameters $D_{1}, \ldots, D_{8}$ which in the simplest case are equal and can vary with some integral power $m$ of energy.

Figure 6 shows the survival probability as a function of neutrino energy $E_{\nu}$ and zenith angle for neutrinos created in the Earth atmosphere. The pattern at lower energies corresponds to "standard oscillations" (without taking into account matter effects), the pattern at higher energies would be expected for non-standard oscillations due to the violation of Lorentz invariance with $\Delta c / c=10^{-27}$.

\subsection{Environmental and marine sciences}

The construction of neutrino telescopes in deep ice or deep water provides opportunities for long-term, real-time measurements in these environments that are unique and therefore of utmost interest to a variety of scientific disciplines beyond astroparticle physics, such as geology and geophysics, marine biology, oceanography or environmental sciences. Owing to the larger construction flexibility in water and to the richness of marine phenomena, corresponding activities are mostly concentrated on marine sites.

Multidisciplinary observatories are associated to all current Mediterranean deep-sea neutrino telescope projects (see [100, 101] and [102] and references therein). For KM3NeT, a 
dedicated interface to such instrumentation is integral part of the planning [103], and a close cooperation with the European Multidisciplinary Seafloor Observatory (EMSO) project [104] has been established. It is expected that deep-sea data will be taken by a series of instruments that are connected to the KM3NeT deep-sea cable network; in conjunction, also the neutrino telescope data themselves will be useful (e.g. light measurements for bioluminescence studies, calibration data for acoustic and water current measurements).

Issues to be addressed with these data are amongst others [103]:

- Investigation of deep-sea phenomena such as internal waves and short time-scale oscillations in the water column, which are relevant for understanding the physical processes of the ocean and their effects on the distribution of suspended geological, chemical and biological materials;

- Real-time tracking of bio-acoustic emissions or vertical migrations of organisms;

- Long-term monitoring of the ocean margin environment around Europe;

- Studies of oceanic processes and the land-ocean-atmospheric interactions. Geological records show that the ecosystem of the Mediterranean Sea amplifies climatologic variations, making it an ideal test bed for climate studies.

In addition, contributions to hazard warning systems (in particular related to tsunamis) are conceivable, even though problems related to reliability standards, data access and usage rights need to be clarified.

In the South Polar ice, the spectrum of multidisciplinary investigations is limited compared to Ocean detectors, but nevertheless manifold. Light propagation in deep ice is affected by remnant air bubbles at shallow depths [105] and by layered impurities from dust which are due to climatic effects or volcano eruptions [106] - see Fig. 19 in Sect. 4.3. Motivated by these findings, a project called DeepIce was proposed in 1999 to the US National Science Foundation. It was suggested to join efforts of astroparticle physicists, glaciologists, seismologists, geophysicists and biologists to investigate and exploit the features of glacial ice at or near the South Pole. The project was not funded, but many of its ideas have been pursued further and have led to relevant results, e.g. on the propagation of acoustic and radio waves in ice (see also Sect. 7), on glaciology, climate research and volcanology. One legacy of DeepIce is an optical dust-logger, which reads out concentrations of dust particles and volcanic ash in the $2.5 \mathrm{~km}$ deep water-filled boreholes with a depth resolution of about $1 \mathrm{~mm}$ [107]. These dust logs, together with similar results from Greenland ice, have revealed a relationship between abrupt climate changes and faint volcanic fallout layers and have permitted for reconstructions of dust and paleowind records of high quality. The detailed investigation of the IceCube dust logs have provided an unique characterisation of Polar deep ice and make the South Pole a leading candidate for a next US deep-ice core project.

Moreover, the thermistors and microinclinometers installed in IceCube allow for studying the shear strain rate of a large volume of ice in three dimensions as a function of stress, impurity content and temperatures down to $-35^{\circ} \mathrm{C}$. Last but not least, we mention an idea on studying biology under extreme conditions which also evolved from DeepIce: a quantitative model of how micron-sized microbes could live in liquid veins at triple junctions of ice grains and how they could be detected [108]. 


\section{Detection Principles}

The classical operation of neutrino telescopes underground, underwater and in deep ice is recording upward-going muons generated in charged current muon neutrino interactions. The upward signature guarantees the neutrino origin of the muon since no other known particle can traverse the Earth. Neutrino telescopes need to be situated at more than a kilometre depth in order to suppress downward-moving muons which may be misreconstructed as upward-moving ones (Fig. 7). Apart from these, only one irreducible background to extraterrestric neutrinos remains: neutrinos generated by cosmic ray interactions in the Earth's atmosphere ("atmospheric neutrinos"). This background cannot be reduced by going deeper. On the other hand, it provides a standard calibration source and a reliable proof of principle.

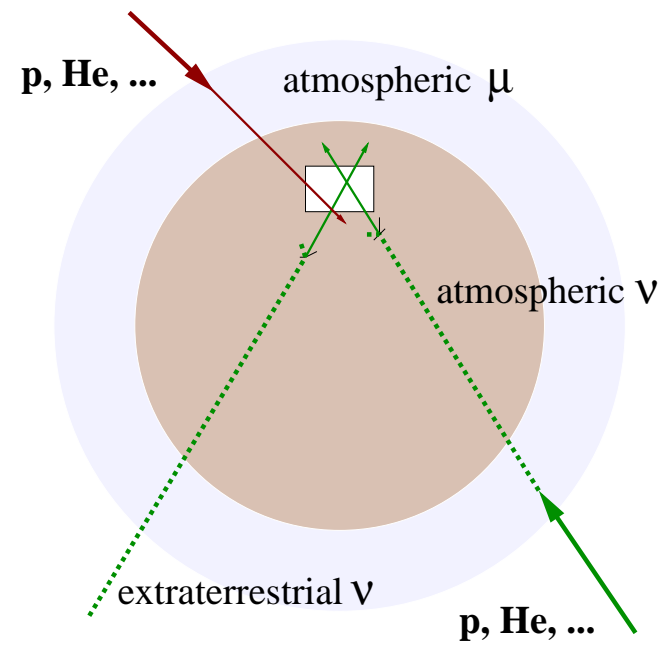

Figure 7. Sources of muons in deep underwater/ice detectors. Cosmic nuclei - protons (p), $\alpha$ particles (He), etc. - interact in the Earth atmosphere (light-coloured). Sufficiently energetic muons produced in these interactions ("atmospheric muons") can reach the detector (white box) from above. Upward-going muons must have been produced in neutrino interactions.

Underwater/ice neutrino telescopes consist of an array of photomultiplier tubes (PMTs) housed in transparent pressure spheres which are spread over a large volume in oceans, lakes or glacial ice. The PMTs record arrival time and amplitude, sometimes even the full waveform, of Cherenkov light emitted by muons or particle cascades. In most designs the spheres are attached to strings which - in the case of water detectors - are moored at the ground and held vertically by buoys. The typical PMT spacing along a string is $10-20 \mathrm{~m}$, and the distance between adjacent strings $60-150 \mathrm{~m}$. The spacing is thus by orders of magnitude larger than in an underground detector like Super-Kamiokande. This allows for covering large volumes, but makes the detector practically blind with respect to events with energies below about $10 \mathrm{GeV}$.

\subsection{Neutrino interactions}

At neutrino energies $E_{\nu}$ above some $10 \mathrm{GeV}$, as relevant for this review, charged-current (anti)neutrino-nucleon reactions are dominated by deep-inelastic scattering, ${ }^{C} \vec{\nu}_{\ell} N \rightarrow \ell^{\mp} X$ (charged current, CC) or $\stackrel{c}{\nu}_{\ell} N \rightarrow \stackrel{c}{\nu}_{\ell} X$ (neutral current, NC), with $\ell=e, \mu, \tau$. The leadingorder differential cross section for the most important process, $\nu_{\mu} \mathrm{CC}$ reactions, is

$$
\frac{\mathrm{d}^{2} \sigma_{\nu}^{(-)} \rightarrow \mu^{\mp} X}{\mathrm{~d} x \mathrm{~d} y}=\frac{2 G_{F}^{2} M_{N} E_{\nu}}{\pi} \cdot\left(\frac{M_{W}^{2}}{Q^{2}+M_{W}^{2}}\right)^{2} \cdot x \begin{cases}d\left(x, Q^{2}\right)+(1-y)^{2} \bar{u}\left(x, Q^{2}\right) & \text { for } \nu \\ (1-y)^{2} u\left(x, Q^{2}\right)+\bar{d}\left(x, Q^{2}\right) & \text { for } \bar{\nu}\end{cases}
$$


where $x$ and $y$ are the Bjorken scaling variables ( $x$ being the fraction of the nucleon momentum carried by the struck quark, $y$ the fraction of the incoming neutrino energy transferred to the hadronic system $X), Q^{2} \approx 2 x y E_{\nu} M_{N}$ is the negative four-momentum transfer squared, $G_{F}$ is the Fermi constant and $M_{N}$ and $M_{W}$ are the nucleon and the $W$ mass, respectively (see [109 for more details). The parton distributions $d\left(x, Q^{2}\right)$ and $u\left(x, Q^{2}\right)$ represent the sums of all $d$-type and $u$-type quark flavours, respectively; likewise $\bar{d}\left(x, Q^{2}\right)$ and $\bar{u}\left(x, Q^{2}\right)$ are the corresponding antiquark distributions. These functions have been measured in fixed-target experiments and at HERA (see [110] and references therein).

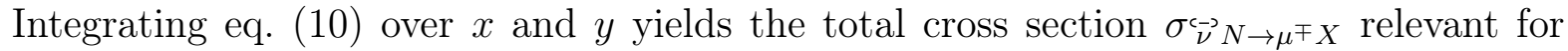
neutrino telescope observations. For $E_{\nu} \lesssim 10 \mathrm{TeV}$ we have $\left\langle Q^{2}\right\rangle \ll M_{W}^{2}$; in this regime the cross section is linear in $E_{\nu}$ to a good approximation, with a value of $\sigma \approx 10^{-35} \mathrm{~cm}^{2}$ at $E_{\nu}=1 \mathrm{TeV}$. For higher energies, the propagator term and the QCD-induced $Q^{2}$ dependence of the parton distributions result in a slower rise, roughly proportional to $E_{\nu}^{0.4}$. The cross sections resulting from a recent analysis [11] for CC and $\mathrm{NC}$ reactions and their uncertainties are shown in Fig. 8. Note that the cross section is per nucleon and is different for neutrinos and antineutrinos; for isoscalar targets, the cross sections roughly have ratio $\nu: \bar{\nu}=2: 1$, whereas they are approximately equal for proton targets. The uncertainties at large neutrino energies are mainly caused by the lack of experimental constraints on the parton distributions at $x \lesssim 10^{-5}$.
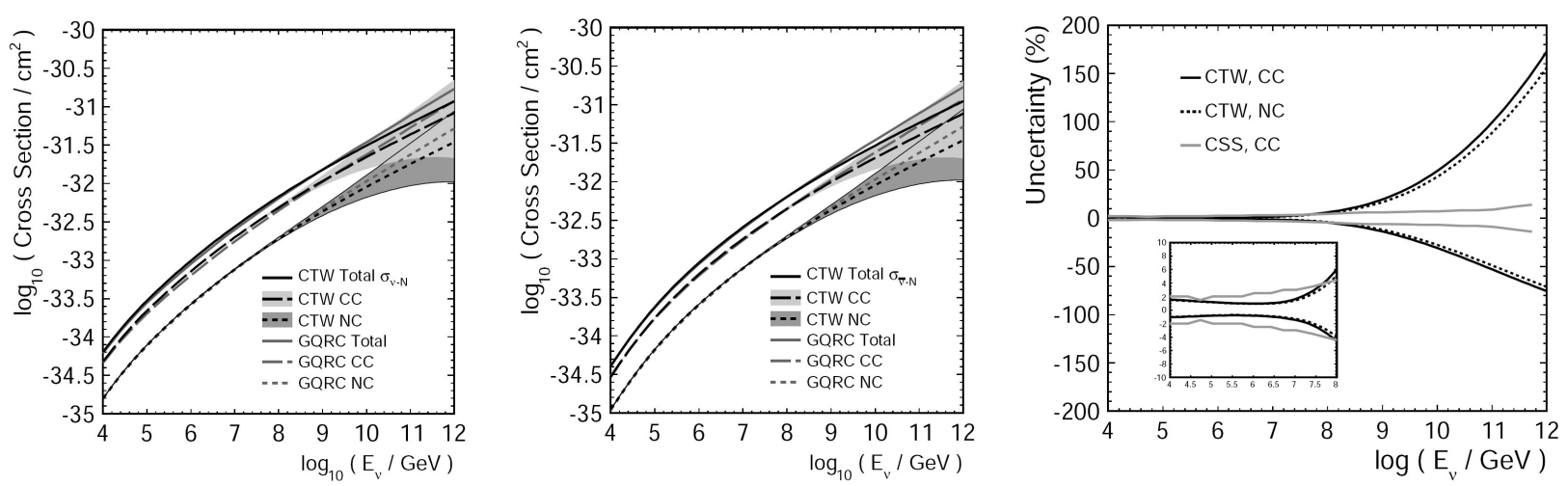

Figure 8. Total cross sections for neutrino (left) and antineutrino (middle) scattering on isoscalar nucleons. Shown are results of two different analyses, both for charged current (CC) and neutral current (NC) scattering. In the right panel, the relative uncertainties in both analyses are indicated. Figures taken from [111] (CTW); shown are also results from [112] (GQRC) and [113] (CSS).

The final state lepton follows the initial neutrino direction with a mean square root mismatch angle $\theta$ decreasing with the square root of the neutrino energy [21]:

$$
\sqrt{\left\langle\theta^{2}\right\rangle} \approx \frac{1.5^{\circ}}{\sqrt{E_{\nu}[\mathrm{TeV}]}} .
$$

In [114, the parameterisation $\langle\theta\rangle \approx 0.7^{\circ} /\left(E_{\nu}[\mathrm{TeV}]\right)^{0.6}$ is given for the average angle. On the one hand, this principally allows for source tracing with CC muon neutrino reactions, but on the other hand represents an intrinsic kinematic limit to the ultimate angular resolution, 
which is slightly worse than for high-energy gamma-ray astronomy and orders of magnitude worse than for conventional astronomy.

For a CC reaction of a muon neutrino with energy $E_{\nu}$, we define $P_{\nu \rightarrow \mu}\left(E_{\nu}, E_{\mu}^{\min }\right)$ to be the probability to produce a muon which reaches the detector with an energy exceeding the minimum detectable energy, $E_{\mu}^{\mathrm{min}}$. This probability depends on the cross section $\mathrm{d} \sigma_{\nu N \rightarrow \mu X}\left(E_{\nu}, E_{\mu}\right) / \mathrm{d} E_{\mu}$ and the effective muon range $R_{\text {eff }}$, which is defined as the range after which the muon energy has decreased to $E_{\mu}^{\min }[21]$ :

$$
P_{\nu \rightarrow \mu}\left(E_{\nu}, E_{\mu}^{\min }=1 \mathrm{GeV}\right)=\frac{\rho}{M_{N}} \int_{E_{\mu}^{\min }}^{E_{\nu}} \mathrm{d} E_{\mu} \frac{\mathrm{d} \sigma_{\nu N \rightarrow \mu X}\left(E_{\nu}, E_{\mu}\right)}{\mathrm{d} E_{\mu}} \cdot R_{\mathrm{eff}}\left(E_{\mu}^{\min }, E_{\mu}\right)
$$

with $\rho$ being the density of the target material and $M_{N}$ the nucleon mass. For water and $E_{\mu}^{\min }=1 \mathrm{GeV}$ the following approximation holds [5]:

$$
P_{\nu \rightarrow \mu}\left(E_{\nu}, E_{\mu}^{\min }\right)= \begin{cases}1.3 \times 10^{-6} \cdot\left(E_{\nu} / 1 \mathrm{TeV}\right)^{2.2} & \text { for } E_{\nu}<1 \mathrm{TeV} \\ 1.3 \times 10^{-6} \cdot\left(E_{\nu} / 1 \mathrm{TeV}\right)^{0.8} & \text { for } E_{\nu}>1 \mathrm{TeV}\end{cases}
$$

This implies that a neutrino telescope can detect a muon neutrino with $1 \mathrm{TeV}$ energy with a probability of about $10^{-6}$ if the telescope is on the neutrino's path.

For a neutrino flux $\Phi_{\nu}\left(E_{\nu}, \vartheta\right)=\left[\mathrm{d}^{4} N_{\nu} /\left(\mathrm{d} E_{\nu} \mathrm{d} t \mathrm{~d} A \mathrm{~d} \Omega\right)\right] \cdot \Delta \Omega$ arriving from a solid angle region $\Delta \Omega$ at zenith $\vartheta$, the number of events recorded by a detector with area $A$ within a time $T$ is given by

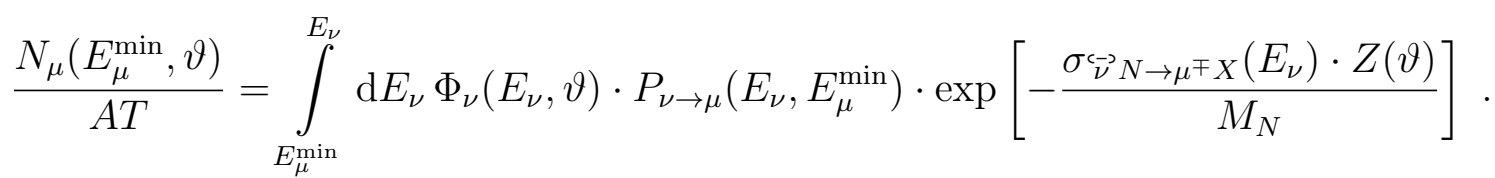

Here $Z(\vartheta)$ is the matter column density in the Earth crossed by the neutrino, in $\mathrm{g} / \mathrm{cm}^{2}$. For sub-TeV energies, absorption in the Earth is negligible and the exponential term is close to unity; for larger neutrino energies, absorption becomes relevant (see Fig.99).

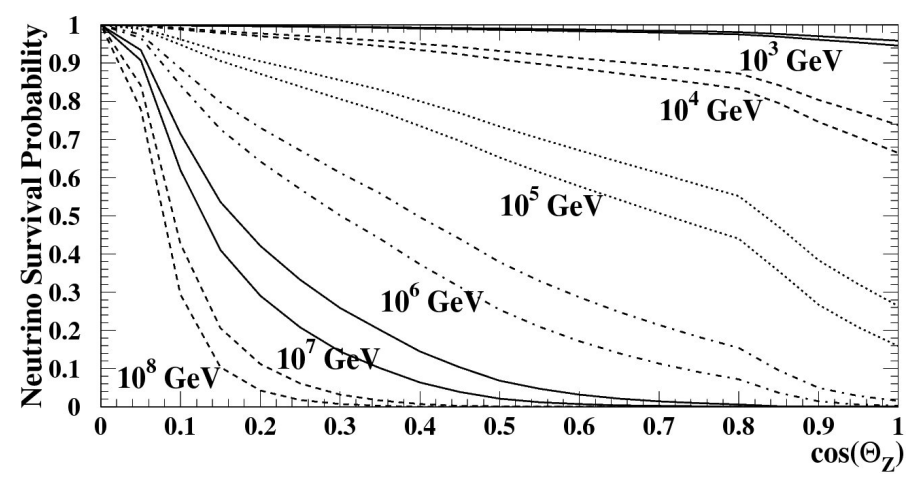

Figure 9. Transmission probability through the Earth for neutrinos of different energies, as a function of the zenith angle. For each energy, the upper line is for CC interactions and the lower one for $\mathrm{CC}$ and $\mathrm{NC}$ interactions. Figure taken from [115].

The fraction of the neutrino energy carried by the final-state lepton is given by $1-y$. For lower energies, where $\left\langle Q^{2}\right\rangle \ll M_{W}^{2}$, integration of eq. 10 yields $1 / 4<\langle y\rangle<1 / 2$, depending 
on the relative contribution of quarks and antiquarks. For larger $E_{\nu}$, the dependence of the propagator term on $Q^{2} \approx 2 x y M_{N} E_{\nu}$ reduces the mean $y$ (see Fig. 10). The muon in $\nu_{\mu}$ $\mathrm{CC}$ reactions on average thus carries the major fraction of the neutrino energy, whereas the hadronic cascade has lower energy.

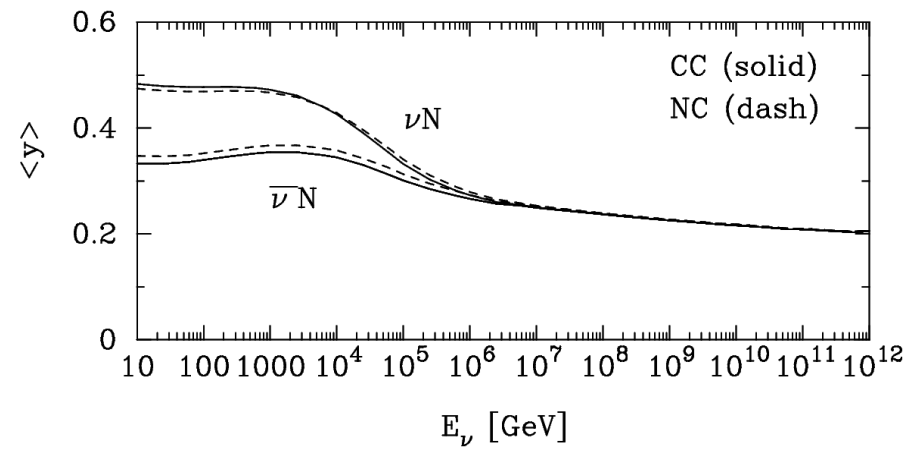

Figure 10. Average $y$ as a function of neutrino energy, for CC (solid lines) and $\mathrm{NC}$ (dashed) reactions. Figure taken from [116].

\subsection{Cherenkov light}

Charged particles moving faster than the speed of light in a medium with index of refraction $n$, i.e. $v>c / n$, emit Cherenkov light under the characteristic angle

$$
\cos \theta_{c}=\frac{1}{\beta \cdot n},
$$

where $\beta=v / c$ and $n$ depends on the frequency $\nu$ of the emitted photons, $n=n(\nu)$. The spectral distribution of Cherenkov photons per path length of an emitting particle with charge $\pm z e$ is given by

$$
\frac{\mathrm{d} N}{\mathrm{~d} x \mathrm{~d} \lambda}=\frac{2 \pi \cdot z^{2} \alpha}{\lambda^{2}} \cdot\left(1-\frac{1}{\beta^{2} \cdot n^{2}}\right)
$$

with $\alpha$ being the fine structure constant. The total amount of released energy per particle path length is obtained by multiplying eq. (16) with $E_{\gamma}=h \nu$ and integrating over $\nu$ :

$$
-\left(\frac{\mathrm{d} E}{\mathrm{~d} x}\right)_{c}=\frac{2 \pi z^{2} \alpha h}{c} \int_{\beta \cdot n(\nu) \geq 1} \nu\left(1-\frac{1}{\beta^{2} \cdot n^{2}(\nu)}\right) \mathrm{d} \nu .
$$

For $\lambda=550 \mathrm{~nm}$ the index of refraction for water is $n \approx 1.33$, yielding about $400 \mathrm{eV} / \mathrm{cm}$, or approximately 200 Cherenkov photons per $\mathrm{cm}$ in the transparency window of water, i.e. for wavelengths $400 \mathrm{~nm} \leq \lambda \leq 700 \mathrm{~nm}$. The average Cherenkov angle of these photons is $\theta_{c} \approx 43^{\circ}$.

\subsection{Light propagation and detection}

The propagation of light in water is governed by absorption and scattering, which both depend on the wavelength $\lambda$. In the first case the photon is lost, in the second case it changes its direction. Scattering effectively delays the propagation of photons between the points of emission and detection. The parameters generally chosen to describe these phenomena are: 
1. The absorption length $L_{a}(\lambda)$ - or the absorption coefficient $a(\lambda)=1 / L_{a}$ - describes the exponential decrease of the number $N$ of non-absorbed photons as a function of photon path length $r, N=N_{0} \cdot \exp \left(-r / L_{a}\right)$.

2. The scattering length $L_{b}(\lambda)$ and scattering coefficient $b(\lambda)=1 / L_{b}$, defined in analogy to $L_{a}(\lambda)$ and $a(\lambda)$.

3. The scattering function $\chi(\theta, \lambda)$, i.e. the distribution in scattering angle $\theta$.

4. Instead of the "geometrical" scattering length $L_{b}(\lambda)$ one frequently uses the effective scattering length $L_{\text {eff }}=L_{b} /(1-\langle\cos \theta\rangle)$, where $\langle\cos \theta\rangle$ is the mean cosine of the scattering angle. $L_{\text {eff }}$ "normalises" scattering lengths for different distributions $\chi(\theta, \lambda)$ of the scattering angle to the extreme case $\langle\cos \theta\rangle=0$, i.e. $L_{\text {eff }}$ can be interpreted as isotropisation length. For $\langle\cos \theta\rangle \approx 0.8-0.95$, as for all media considered here, photon delay effects in media with the same $L_{\text {eff }}$ are approximately the same.

In Table 1, typical parameter values for Lake Baikal, oceans and the Antarctic ice are summarised (see [117] for references). All values are given for the wavelength where they are maximal.

\begin{tabular}{|l|c|c|}
\hline Site & $L_{a}[\mathrm{~m}]$ & $L_{\text {eff }}[\mathrm{m}]$ \\
\hline Lake Baikal, 1 km depth & 22 & $\begin{array}{c}150-400 \\
\text { (seasonal variations) }\end{array}$ \\
\hline Ocean, $>1.5 \mathrm{~km}$ depth & $\begin{array}{c}40-70 \text { (depends } \\
\text { on site and season) }\end{array}$ & $\begin{array}{c}200-400 \text { (depends } \\
\text { on site and season) }\end{array}$ \\
\hline Polar ice, $1.5-2.0 \mathrm{~km}$ depth & $\approx 95$ (average) & $\approx 20$ (average) \\
\hline Polar ice, $2.2-2.5 \mathrm{~km}$ depth & $\approx 150$ (average) & $\approx 40$ (average) \\
\hline
\end{tabular}

Table 1. Absorption length and effective scattering length for deep lake and ocean water and for deep ice.

Scattering and absorption in water and ice are measured with artificial light sources. The scattering coefficient, to a large part due to particulate matter, changes with wavelength less pronouncedly than the absorption coefficient (see Fig. 20 for ice). In water, the depth dependence over the vertical dimensions of a neutrino telescope is small, but parameters may vary with time, due to transient water inflows loaded with bio-matter or dust, or due to seasonal changes in water parameters. They must therefore be permanently monitored. In glacial ice at the South Pole, the situation is different. The parameters are constant in time but strongly depend on depth (see below).

Strong absorption leads to reduced photon collection, strong scattering deteriorates the time information which is essential for the precise reconstruction of tracks and showers (see below).

Efficient and accurate detection and reconstruction of neutrino events requires light detection with a sensitivity at the single-photon level and a measurement of the arrival time 
with nanosecond precision. Furthermore, the integrated photo-sensitive area, multiplied with the single-photon detection efficiency, is the prime parameter determining the sensitivity of the neutrino telescope and is to be maximised. Currently, PMTs [118, 119] are the only devices matching these constraints at an affordable price. Relevant parameters are the photocathode size (i.e. the diameter of the tube); the quantum efficiency (probability that an incoming photon causes emission of a photo-electron) and its spectral dependence; the collection efficiency (probability that a photo-electron causes a measurable signal); the transit time spread (jitter of the delay between photon interaction and output signal); the gain (electric amplification, i.e. average number of electrons in the signal of one photo-electron).

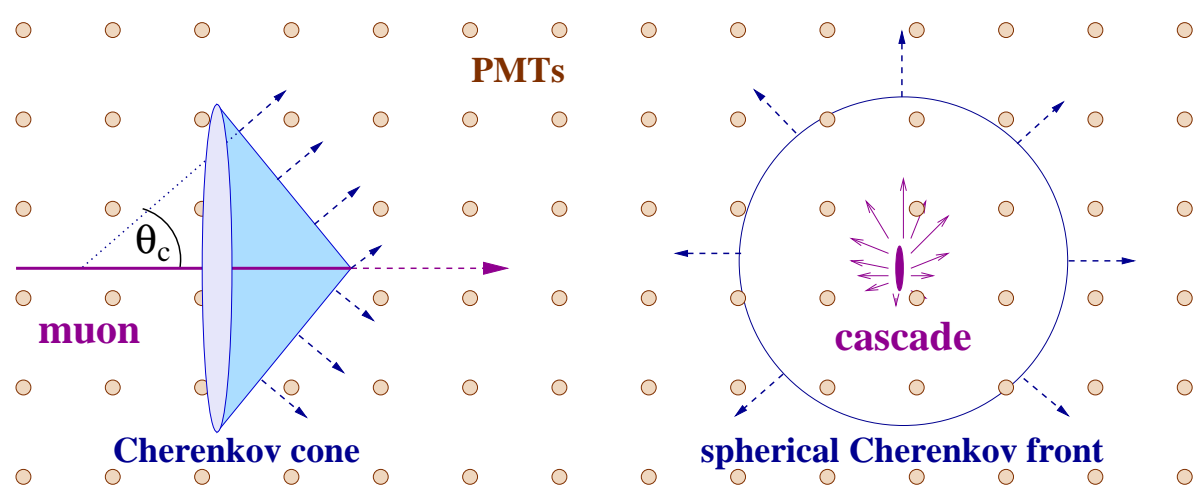

Figure 11. Detection principles for muon tracks (left) and cascades (right) in underwater/ice detectors. Note that the Cherenkov light emission by cascades is peaked at the Cherenkov angle $\theta_{c}$ with respect to the cascade axis but has a wide distribution covering the full solid angle.

\subsection{Detection of muon tracks and cascades}

Figure 11 sketches the two basic detection modes of underwater/ice neutrino telescopes. In the following, $N$ denotes the target nucleon and $X$ the hadronic final state. CC muon neutrino interactions, ${ }^{\stackrel{\nu}{\nu}}{ }_{\mu} N \rightarrow \mu^{\mp} X$ produce a muon track (left; an IceCube event display of a $10 \mathrm{TeV}$ muon is shown in Fig. 12), whereas other neutrino reaction types cause hadronic and/or electromagnetic cascades (right). This is in particular true for NC reactions $\left({ }^{c} \vec{\nu} N \rightarrow\right.$ ${ }^{`} \vec{\nu} X$, hadronic cascade) or CC reactions of electron neutrinos ( ${ }^{`} \vec{\nu}_{e} N \rightarrow e^{\mp} X$, overlapping hadronic and electromagnetic cascades). CC tau neutrino interactions, ${ }^{\complement} \vec{\nu}_{\tau} N \rightarrow \tau^{\mp} X$, can have either signature, depending on the $\tau$ decay mode. In most astrophysical models, neutrinos are produced with a flavour ratio $\nu_{e}: \nu_{\mu}: \nu_{\tau} \approx 1: 2: 0$. Over cosmic distances, oscillations turn this ratio to $\nu_{e}: \nu_{\mu}: \nu_{\tau} \approx 1: 1: 1$ (cf. Sect.2.2.3), which means that almost $2 / 3$ of the charged current interactions initiate cascades only.

\subsubsection{Muon tracks}

The most precise measurement of the neutrino direction is achieved for charged current muon (anti)neutrino interactions; this channel is therefore central to all investigations of astrophysical neutrino sources. The muon direction, and also its energy, are reconstructed from the Cherenkov cone accompanying upward-going muons. The upward signature guarantees the neutrino origin of the muon since no other particle can cross the Earth. The 


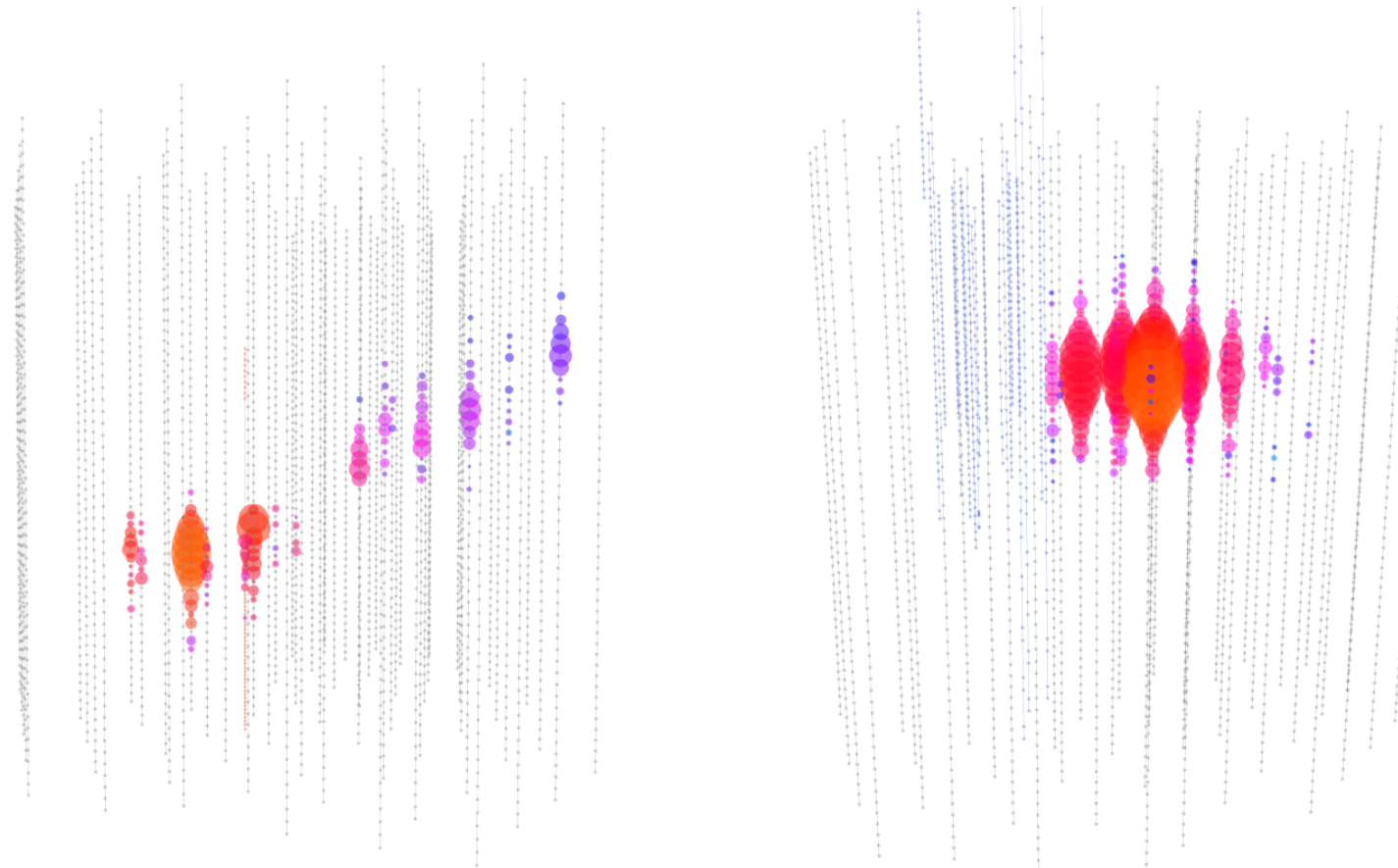

Figure 12. Event displays of a muon (left) and a cascade (right) event recorded in IceCube. The coloured circles indicate the photomultipliers hit; their sizes correspond to the numbers of photons detected. The colour code visualises the hit times, with red hits earliest and violet ones latest. The reconstructed zenith angle and energy of the muon are $117^{\circ}$ and $20 \mathrm{TeV}$, respectively; the reconstructed cascade energy is $175 \mathrm{TeV}$.

effective target volume considerably exceeds the actual detector volume due to the large range of muons (about $0.96 \times 10^{5} \mathrm{~g} / \mathrm{cm}^{2}\left(1.04 \times 10^{5} \mathrm{~g} / \mathrm{cm}^{2}\right)$ at $300 \mathrm{GeV}$ and $14 \times 10^{5} \mathrm{~g} / \mathrm{cm}^{2}$ $\left(12 \times 10^{5} \mathrm{~g} / \mathrm{cm}^{2}\right)$ at $100 \mathrm{TeV}$ in water/ice (standard rock), logarithmically rising towards larger energies [120]; $10^{5} \mathrm{~g} / \mathrm{cm}^{2}$ corresponds to $1 \mathrm{~km}$ in pure water).

The muon loses energy mainly via ionisation, pair production, bremsstrahlung and photonuclear reactions. The energy loss can be parameterised by [21, 120]

$$
-\frac{\mathrm{d} E_{\mu}}{\mathrm{d} x}=a+b \cdot E_{\mu}
$$

where $a$ and $b$ are weakly energy dependent. For water, the ionisation loss is roughly given by $a=2 \mathrm{MeV} / \mathrm{cm}$. The energy loss from pair production, bremsstrahlung and photonuclear reactions becomes dominant at energies beyond $1 \mathrm{TeV}$, where it is approximately linear in $E_{\mu}$; for water and $E_{\mu}=100 \mathrm{TeV}$ it is given by $b_{\text {tot }}=b_{\text {pair }}+b_{\text {brems }}+b_{\text {phonuc }}=$ $(1.7+1.2+0.6) \times 10^{-6} \mathrm{~g} \mathrm{~cm}^{-2}=3.5 \times 10^{-6} \mathrm{~g} \mathrm{~cm}^{-2}[120]$. Note that all energy loss mechanisms producing relativistic charged particles - in particular $e^{ \pm}$from pair production and conversion of bremsstrahlung photons - also lead to additional Cherenkov light.

For the reconstruction of a muon track, its Cherenkov photons need to be measured at least at five positions. In reality, because of timing inaccuracies due to scattering (mostly in ice) and to light background from radioactive decays (deep sea) and bioluminescence (lakes 
and deep sea), a reliable reconstruction requires typically 10 or more PMT signals.

Underwater/ice telescopes are optimised for the detection of muon tracks at energies of the order $\mathrm{TeV}$ or above, for the following reasons:

1. The flux of neutrinos from cosmic accelerators is expected to be harder (generic shape $E_{\nu}^{-2}$ ) than that of atmospheric neutrinos $\left(E_{\nu}^{-3.7}\right)$, yielding a better signal-tobackground ratio at higher energies.

2. Neutrino cross section and muon range increase with energy. The larger the muon range, the larger the effective detector volume (even though this argument must be used with care since a muon that has lost most of its energy when reaching the detector may be discarded due to energy-dependent selection cuts).

3. The mean angle between muon and neutrino decreases with energy (see eq. (11)), resulting in better source tracing and signal-to-background ratio at high energy.

4. For energies above a $\mathrm{TeV}$, the light emission increases linearly with $E_{\mu}$, providing an estimate for the muon energy with an accuracy of $\sigma\left(\log E_{\mu}\right) \approx 0.3$. By unfolding procedures, a muon energy spectrum can be translated into a neutrino energy spectrum.

Muons which have been generated by cosmic ray interactions in the atmosphere above the detector and punch through the water or ice down to the detector outnumber neutrinoinduced upward-moving muons by several orders of magnitude (about $10^{6}$ at $1 \mathrm{~km}$ depth and $10^{4}$ at $4 \mathrm{~km}$ depth) and have to be removed by careful up/down discrimination.

At energies above a few hundred $\mathrm{TeV}$, where the Earth becomes opaque even to neutrinos, neutrino-generated muons arrive preferentially from directions close to the horizon, at EeV energies essentially only from the upper hemisphere (Fig.99). The high energy deposition of muons from $\mathrm{PeV}-\mathrm{EeV}$ extraterrestrial neutrinos provides a handle to distinguish them from downward-going atmospheric muons (that have a spectrum decreasing much more steeply with energy).

\subsubsection{Cascades}

Charged current interactions of electron and, in most cases, tau neutrinos and all neutral current interactions do not lead to high energy muons but to electromagnetic or hadronic cascades. Their length increases only with the logarithm of the cascade energy and is of the order $5-20 \mathrm{~m}$. Cascade events therefore typically need to be contained in the instrumented volume of the detector to be reconstructible ("contained events").

With their small length and a diameter of the order of $10-20 \mathrm{~cm}$, cascades may be considered as quasi point-like compared to the spacing of the PMTs. The effective volume for cascade detection is close to the instrumented volume of the detector. For first-generation neutrino telescopes it is therefore much smaller than that for muon detection. However, for kilometre-scale detectors and moderate energies both can be of the same order of magnitude. The total amount of light is proportional to the cascade energy and thus provides a direct measurement of $E$ for contained cascades, in contrast to muons, for which only $\mathrm{d} E / \mathrm{d} x$ 
can be measured. Therefore, in charged current $\nu_{e}$ interactions, the neutrino energy can be determined with an accuracy of about $30 \%$ (note, however, that this is not true for NC events; also, in $\nu_{\tau} \mathrm{CC}$ events the $\nu_{\tau}$ from the decay of the final-state $\tau$ carries away a sizeable fraction of "invisible" energy). Whereas the energy resolution is much better than for muons, the directional accuracy is worse since the lever arm for fitting the direction is small (for actual values, see below). The irreducible background from atmospheric electron neutrinos is significantly smaller than that of atmospheric muon neutrinos for the muon channel. All this makes the cascade channel particularly interesting for searches for a diffuse, high-energy excess of extraterrestrial over atmospheric neutrinos.

\subsection{Effective area and sensitivity}

The minimum energy of a muon to be recorded depends on the impact point and the impact angle, resulting in a smearing for $E_{\mu}^{\min }$. Even muons outside the geometrical area may fulfil the trigger condition. On the other hand, background suppression usually requires tight cuts during the analysis, so that muons having triggered the detector might be rejected at a later stage. Therefore, the geometrical area $A$ is replaced by the effective muon area $A_{\text {eff }}^{\mu}$ which is calculated from Monte Carlo simulations as follows: $N_{\text {gen }}$ muons are generated perpendicular to an area $A_{\text {gen }}$ significantly larger than the detector cross section. With $N_{\text {passed }}$ muons accepted after all cuts, the effective area is then given by

$$
A_{\text {eff }}^{\mu}=A_{\text {gen }} \cdot \frac{N_{\text {passed }}}{N_{\text {generated }}} .
$$

The effective area depends on $\theta$ and $\phi$, i.e. the muon direction with respect to the detector. Due to the increasing range and light emission, $A_{\text {eff }}^{\mu}$ increases with muon energy. Note that $A_{\text {eff }}^{\mu}$ also depends on the distance between the point where the muons are generated and the detector.

The concept of an effective area can also be applied to neutrinos, taking into account the neutrino nucleon cross section, the muon range and the absorption in the Earth according to Sect. 3.1. Figure 13 shows the effective neutrino area of IceCube as a function of energy and zenith angle. The strong increase with energy is due to the increasing neutrino cross section and to the increasing muon range. The decrease at high energies for large zenith angles is due to neutrino absorption in the Earth.

Due to the small neutrino interaction cross section, the effective neutrino area is many orders of magnitude smaller than the muon effective area. Even cubic-kilometre neutrino telescopes reach only neutrino areas between a few square metres and a few hundred square metres, depending on energy. This has to be compared to several ten thousand square metres typical for air Cherenkov telescopes which detect gamma-ray initiated air showers. A ratio 1:1000 $\left(10 \mathrm{~m}^{2}: 10000 \mathrm{~m}^{2}\right)$ may appear desperately small. However, one has to take into account that Cherenkov gamma telescopes can only observe one source at a time, and that their observations are restricted to Moon-less, clear nights. Neutrino telescopes observe a full hemisphere, 24 hours per day. Therefore, cubic-kilometre detectors reach a flux sensitivity similar to that reached by first-generation Cherenkov gamma telescopes like Whipple and HEGRA [122, 123] for TeV gamma rays, i.e. $\Phi(>1 \mathrm{TeV}) \approx 10^{-12} \mathrm{~cm}^{-2} \mathrm{~s}^{-1}$. 


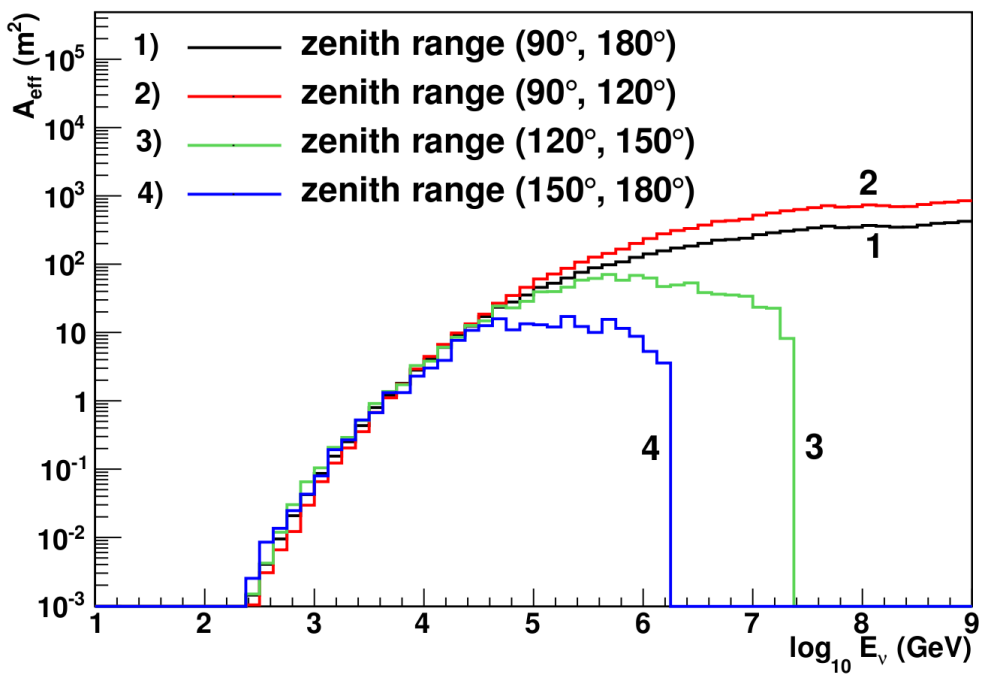

Figure 13. Effective neutrino area of the 40-string configuration of IceCube as a function of energy, averaged over different zenith angle ranges. At energies beyond $10^{4} \mathrm{GeV}$, neutrino absorption $\mathrm{n}$ the Earth becomes important. Figure taken from [121.

\subsection{Reconstruction}

In this section, some relevant aspects of event reconstruction are discussed for the case of muon tracks [124 126]. For cascades, see [127-129]. The reconstruction procedure for a muon track consists of several consecutive steps which are typically:

1. Rejection of noise hits;

2. Simple pre-fit procedures providing a first-guess estimate for the following iterative maximum-likelihood reconstruction;

3. Maximum-likelihood reconstruction;

4. Quality cuts in order to reduce background contaminations and to enrich the sample with signal events. This step is strongly dependent on details of the actual analysis diffuse fluxes at high energies, searches for steady point sources, searches for transient sources etc.

An infinitely long muon track can be described by an arbitrary point $\vec{r}_{0}$ on the track which is passed by the muon at time $t_{0}$, with a direction $\hat{p}$ and energy $E_{0}$ (see Fig. 14). Since the muon in very good approximation propagates with the vacuum speed of light, $c$, photons emitted under the Cherenkov angle $\theta_{c}$ (see eq. (15)) and propagating on a straight path ("direct photons") are expected to arrive at PMT $i$ located at $\vec{r}_{i}$ at a time

$$
t_{\text {geo }}=t_{0}+\frac{\hat{p} \cdot\left(\vec{r}_{i}-\vec{r}_{0}\right)+d \cdot \tan \theta_{c}}{c}
$$

where $d$ is the closest distance between PMT $i$ and the track. The time residual $t_{\text {res }}$ is given by the difference between the measured hit time $t_{\text {hit }}$ and the hit time expected for a direct photon, $t_{\text {geo }}$ :

$$
t_{\mathrm{res}}=t_{\mathrm{hit}}-t_{\mathrm{geo}}
$$




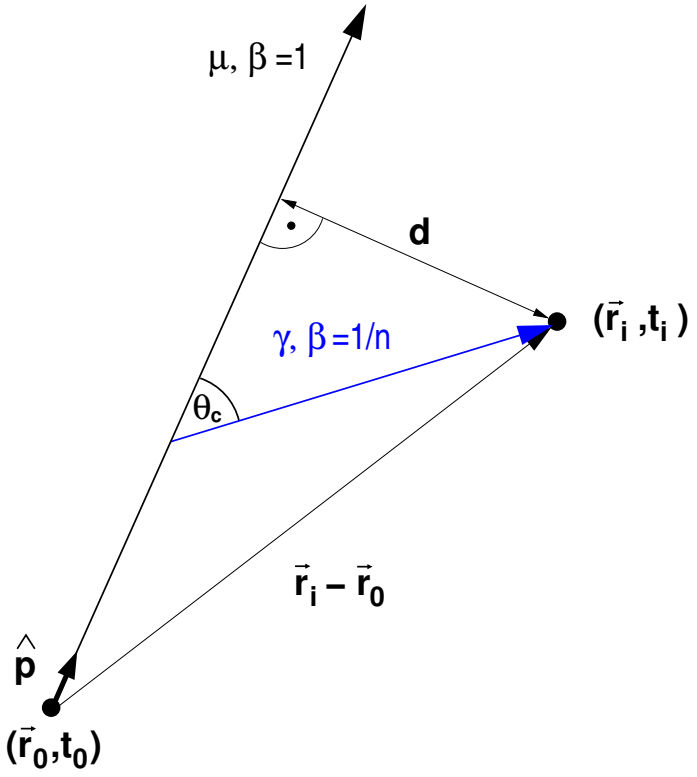

Figure 14. Geometry of muon reconstruction: The muon, propagating with $\beta=1$ in very good approximation, radiates a Cherenkov photon under the angle $\theta_{c}$ (with $\cos \theta_{c}=1 / n$ ) that propagates with $\beta=1 / n$ on a straight line and hits the photon sensor at position $\vec{r}_{i}$. Under these assumptions the hit time can be calculated to be $t_{i}=t_{\text {geo }}$ (see eq. (20p). The time difference $t_{i}-t_{0}$ thus relates the muon track parameters (position $\vec{r}_{0}$, direction $\hat{p}$ ) to the position of the photon hit.

Schematic distributions for time residuals are given in Fig. 15. An unavoidable symmetric contribution around $\Delta_{t}=0$ in the range of a nanosecond comes from the PMT/electronics time jitter, $\sigma_{t}$. An admixture of noise hits to the true hits from a muon track adds a flat pedestal contribution like shown in top right of Fig. 15. Electromagnetic cascades along the track, initiated by bremsstrahlung and pair production, lead to a tail towards larger (and only larger) time residuals (bottom left). Scattering of photons can lead to an even stronger delay of the arrival time (bottom right). These residuals must be properly implemented in the probability density function for the arrival times used in the maximum-likelihood procedure.
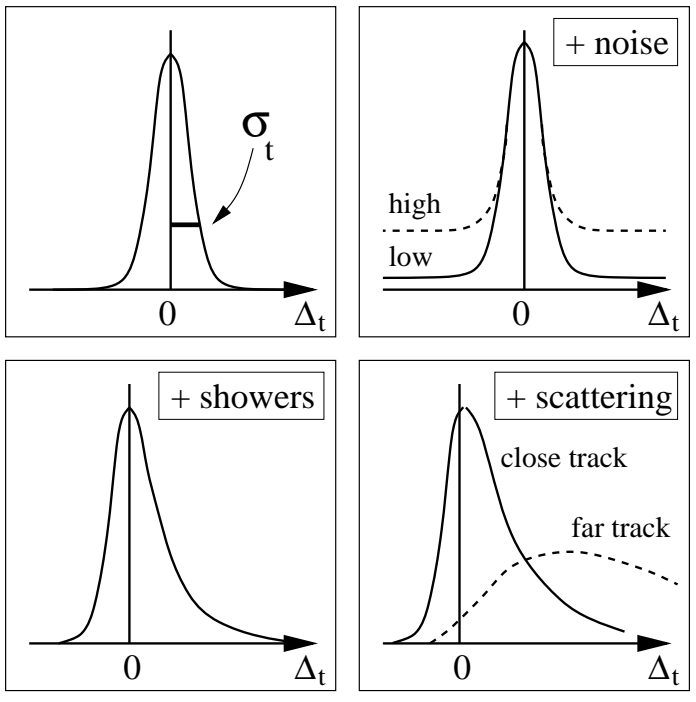

Figure 15. Schematic distributions of arrival times for different cases (see text). 
The simplest likelihood function is based exclusively on the measured arrival times. It is the product of all $N_{\text {hit }}$ probability density functions $p_{i}$ to observe, for a given value of track parameters $\{a\}$, photons at times $t_{i}$ at the location of the PMTs hit:

$$
L_{\mathrm{time}}=\prod_{i=1}^{N_{\mathrm{hit}}} p\left(t_{\mathrm{res}, i} \mid\{a\}\right) .
$$

More complicated likelihood functions include the probability of hit PMTs to be hit and of non-hit PMTs not to be hit, or of the respective amplitudes. Instead of referring only to the arrival time of the first photon for a given track hypothesis and the amplitude for a given energy hypothesis, one may also refer to the full waveform from multiple photons hitting the PMT. For efficient background suppression, the likelihood may also incorporate information about the zenith angular dependence of background and signal (Bayesian probability). The reconstruction procedure finds the best track hypothesis by maximising the likelihood. 


\section{First-Generation Neutrino Telescopes}

An explorative phase of more than two decades was required to solve the technical problems of instrumenting target masses of the order of 10 Megatons in deep ice, fresh or sea water and to overcome the difficulties which these hostile environments pose. Nevertheless, neutrino telescopes of this size have been constructed and successfully operated in all three media. In this Section, the technical setup of these installations is discussed, also with reference to the history of major steps leading to their construction.

\subsection{DUMAND}

The history of underwater neutrino telescopes starts with a project which eventually failed but left an incredibly rich legacy of ideas and technical principles: The DUMAND project. DUMAND stands for Deep Underwater Muon and Neutrino Detector. Its early history is excellently covered in a "Personal history of the DUMAND project" by A. Roberts [130]. At the 1973 International Cosmic Ray Conference (ICRC), a small group of physicists conceived a deep-water detector to clarify puzzles in muon depth-intensity curves. The puzzles faded away, but it was obvious that such a detector could also work for neutrinos. The year 1975 saw the first of a - meanwhile legendary - series of DUMAND Workshops. Soon, the decision was taken to deploy the detector $30 \mathrm{~km}$ off the coast of Big Island, Hawaii, at a depth of $4.8 \mathrm{~km}$.

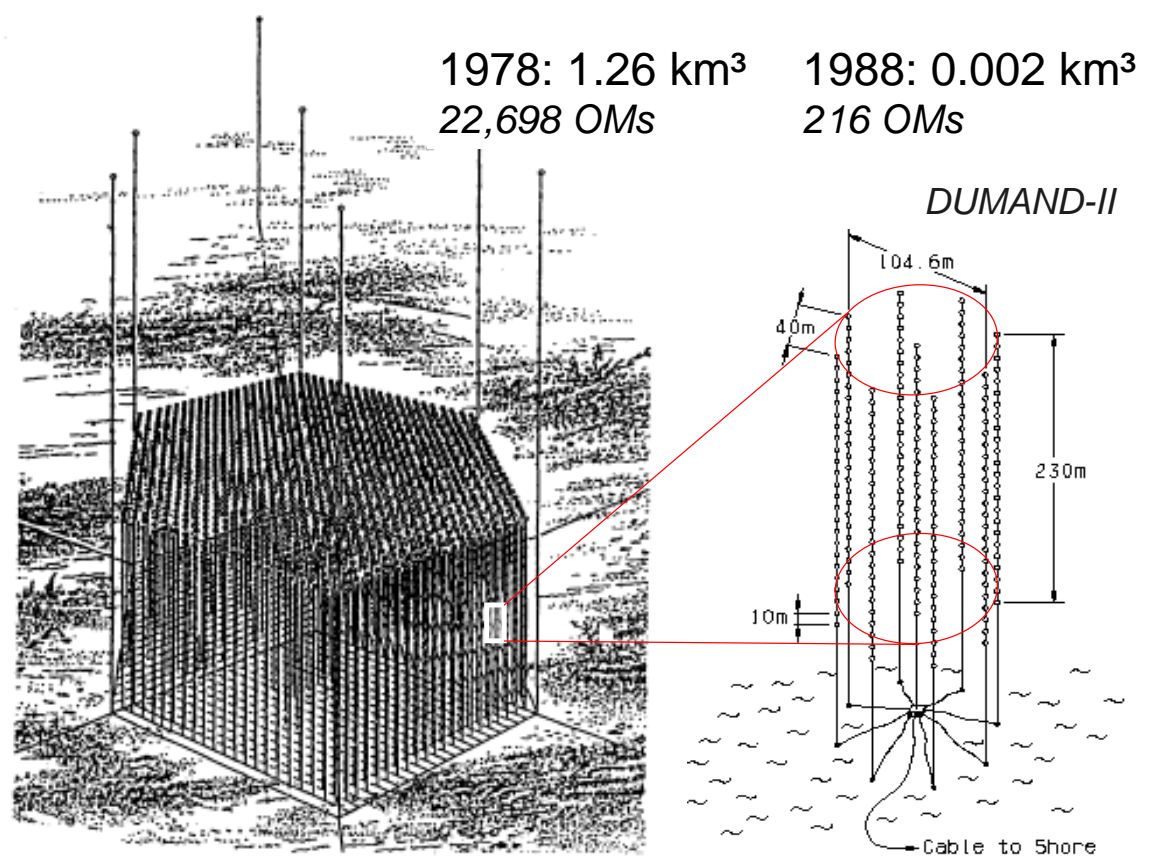

Figure 16. The originally conceived DUMAND cubic-kilometre detector and the 1988 plan for the first-generation underwater neutrino telescope DUMAND-II.

The original idea to construct a cubic-kilometre detector (1978) with more than 20000 large-size photomultipliers (see Fig. 16) was quickly abandoned due to technical and financial reasons. A half-sized configuration (1980) met the same fate, as did a much smaller array with 756 phototubes (1982). The latter design was comparable in size to the meanwhile 
decommissioned AMANDA detector at the South Pole (see Sect. 4.3) and the operating ANTARES telescope in the Mediterranean Sea, close to Toulon (see Sect. 4.4). What finally emerged as a technical project was a 216-phototube version, dubbed DUMAND-II or "The Octagon" (eight strings at the corners of an octagon and one in the centre), $100 \mathrm{~m}$ in diameter and $230 \mathrm{~m}$ in height [131.

Signals on at least three strings are required for full spatial reconstruction of a muon trajectory. Consequently, the sequential deployment was planned to start with TRIAD, a sub-array of three full strings. Unfortunately, pressure housings of the first string developed leaks during deployment in 1993 and soon the communication to shore failed. The "3-string race" between DUMAND, the Baikal project and AMANDA was eventually won by the Baikal collaboration in 1993, whereas the DUMAND project was terminated in 1995.

Ironically, the most sustaining physics result from the DUMAND project has been obtained with a 7-phototube test string deployed for only some hours from a ship [132]. It gives the measured muon intensity as a function of depth and thus returned to the initial idea of the 1973 ICRC.

\subsection{The Baikal neutrino telescope}

The Baikal Neutrino Telescope is installed in the Southern part of Lake Baikal [133, 134]. The distance to shore is $3.6 \mathrm{~km}$, the depth of the lake at this location is $1366 \mathrm{~m}$, the active part of the detector is located at a depth of about $1.1 \mathrm{~km}$.

First site surveys started in 1980. In 1984, a first stationary string was deployed and muons recorded [135]. This was followed by another stationary string in 1986 which was optimised for the detection of magnetic monopoles catalysing proton decay [136, 137].

A new period began with the development of the QUASAR photodetector (see below), which replaced the former $15 \mathrm{~cm}$ flat photomultipliers, and the design of the array NT200 (see Fig. 17, left). The BAIKAL collaboration was not only the first, in 1993, to deploy three strings (as necessary for full spatial reconstruction of muon trajectories), but also reported the first atmospheric neutrino detected underwater (Fig. 17, right).

The central part of the Baikal configuration is NT200, an array of 192 optical modules which was completed in April 1998 and has been taking data since then. The optical modules are carried by eight strings which are attached to an umbrella-like frame. The strings are anchored by weights at the lake floor and held in a vertical position by buoys at various depths. The configuration spans $72 \mathrm{~m}$ in height and $43 \mathrm{~m}$ in diameter. The detector is deployed (or hauled up for maintenance) within a period of about 6 weeks in February to April, when the lake is covered with a thick ice layer providing a stable working platform. It is connected to shore by several copper cables on the lake floor which allow for operation over the full year.

The optical modules are glass spheres equipped with QUASAR-370 phototubes; they are grouped pair-wise along a string. In order to suppress accidental hits from dark noise (about $30 \mathrm{kHz}$ ) and bioluminescence (typically $50 \mathrm{kHz}$ but seasonally raising up to hundreds of $\mathrm{kHz}$ ), the two photomultipliers of a pair are switched in coincidence, defining a channel, with typically only $0.1 \mathrm{kHz}$ noise rate. The basic cell of NT200 consists of a svjaska (Russian for "bundle"), comprising two optical module pairs and an electronics module for time and 

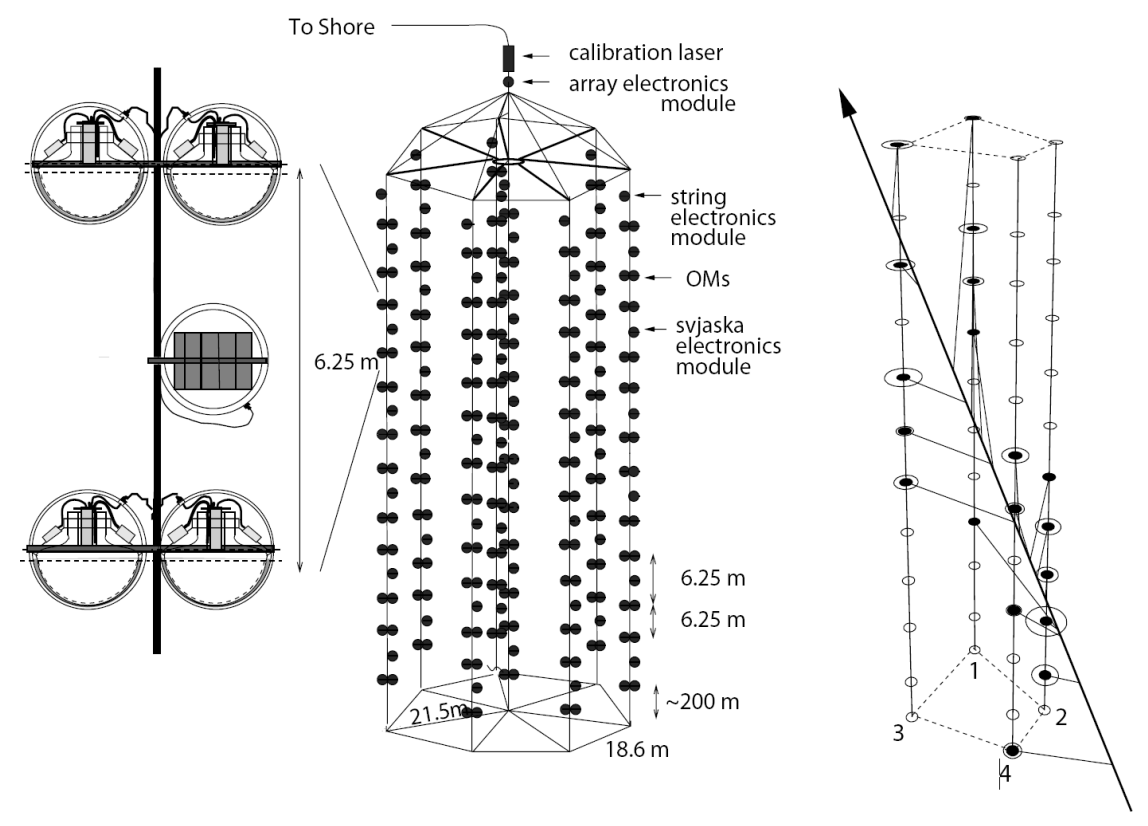

Figure 17. Left: The Baikal Neutrino Telescope NT200. Right: One of the first upward moving muons from a neutrino interaction recorded with the 4 -string stage of the detector in 1996 [138]. The Cherenkov light from the muon is recorded by 19 channels.

amplitude conversion and slow control functions (Fig. 17, left). A majority trigger is formed if $\geq 4$ channels are fired within a time window of $500 \mathrm{~ns}$ (this is about twice the time a relativistic particle needs to cross the NT200 array). Trigger and inter-string synchronisation electronics are housed in an array electronics module at the top of the umbrella frame. This module is less than $100 \mathrm{~m}$ away from the optical modules, allowing for nanosecond synchronisation over copper cable.
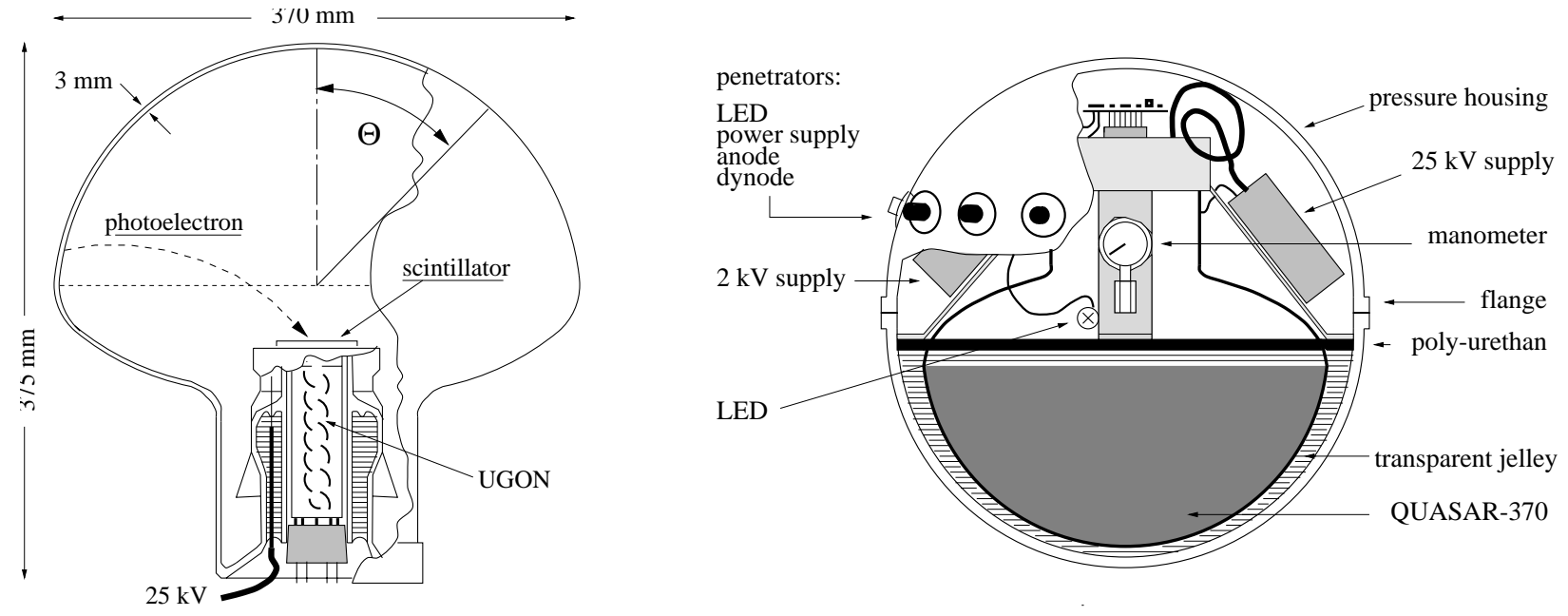

Figure 18. Left: The QUASAR-370 phototube. Right: A full Baikal optical module.

Figure 18 shows the phototube and the full optical module [139]. The QUASAR-370 is a hybrid device. Photoelectrons from a large hemispherical cathode $\left(\mathrm{K}_{2} \mathrm{CsSb}\right)$ with $>2 \pi$ viewing angle are accelerated by $25 \mathrm{kV}$ to a fast, high-gain scintillator which is placed near the centre of the glass bulb. The light from the scintillator is read out by a small conventional 
photomultiplier (type UGON). One photo-electron from the hemispherical photocathode yields typically 20 photoelectrons in the conventional photomultiplier. This high multiplication factor results in an excellent single electron resolution of $70 \%$. Furthermore, the QUASAR-370 is characterised by a small time jitter $(2 \mathrm{~ns})$ and a small sensitivity to the Earth's magnetic field.

The small spacing of modules leads to a comparably low energy threshold of about $15 \mathrm{GeV}$ for muon detection. About 400 upward muon events were collected over 5 years. Still, NT200 could compete with the much larger AMANDA for a while by searching for high energy cascades below NT200, surveying a volume about ten times as large as NT200 itself [140]. In order to improve pattern recognition for these studies, NT200 was fenced in 2005-2007 with three sparsely instrumented outer strings (6 optical module pairs per string). This configuration is named NT200+ [141.

\section{3. $A M A N D A$}

AMANDA (Antarctic Muon And Neutrino Detection Array) used the $3 \mathrm{~km}$ thick ice layer at the South Pole as target and detection medium [142, 143]. AMANDA was located some hundred metres away from the Amundsen-Scott station. Holes of $60 \mathrm{~cm}$ diameter are drilled with pressurised hot water; strings with optical modules are deployed in the molten water which subsequently refreezes. Installation operations at the South Pole are performed in the Antarctic summer, November to February. For the rest of the time, two operators (of a winter-over crew of 25-40 persons in total) maintain the detector, connected to the outside world via satellite communication.

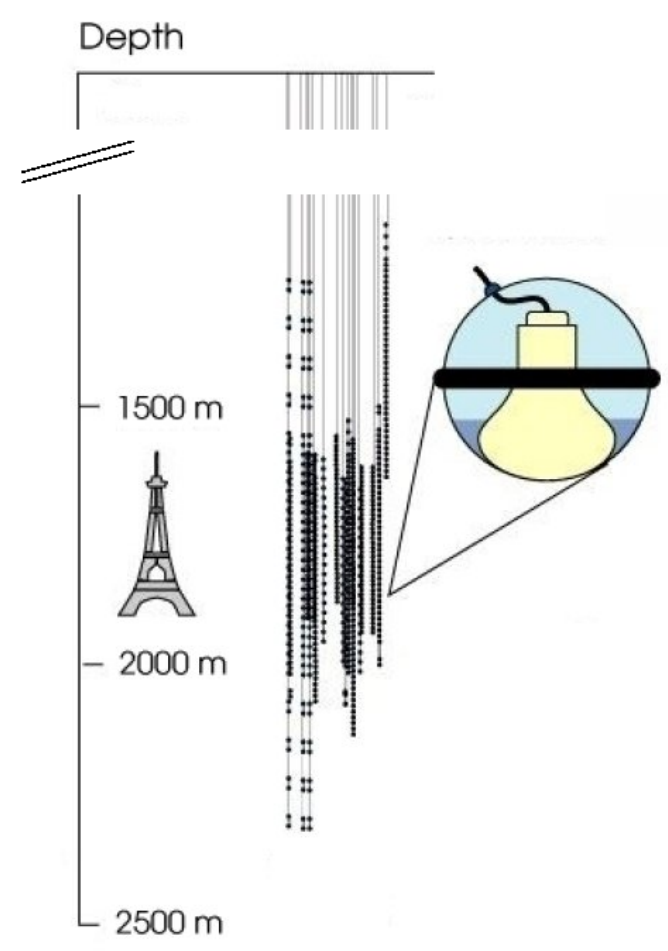

Figure 19. The AMANDA configuration. Three of the 19 strings have been sparsely equipped towards larger and smaller depth in order to explore ice properties, one string got stuck during deployment at too shallow depth and was not used in analyses. The Eiffel tower is shown to scale for size comparison. 
Figure 19 shows the AMANDA configuration. A first test array with 80 optical modules at four strings (not shown) was deployed in 1993/94, at depths between 800 and $1000 \mathrm{~m}$ [105. The effective scattering length $L_{\text {eff }}$ was found to be extremely small, between $40 \mathrm{~cm}$ at $830 \mathrm{~m}$ depth and $80 \mathrm{~cm}$ at $970 \mathrm{~m}$. The scattering is due to air bubbles trapped in the ice and makes track reconstruction impossible. The tendency of the scattering to decrease with depth, as well as results from ice core analyses at other places in Antarctica, suggested that the bubbles should disappear below $1300 \mathrm{~m}$. This expectation was confirmed with a second 4-string array which was deployed in 1995/96. The remaining scattering, averaged over $1500-2000 \mathrm{~m}$ depth, corresponds to $L_{\text {eff }} \approx 20 \mathrm{~m}$ and is assumed to be due to dust. This is still considerably worse than for water but sufficient for track reconstruction [106, 124]. The array was upgraded stepwise until January 2000 and eventually comprised 19 strings with a total of 677 optical modules, most of them at depths between 1500 and $2000 \mathrm{~m}$.
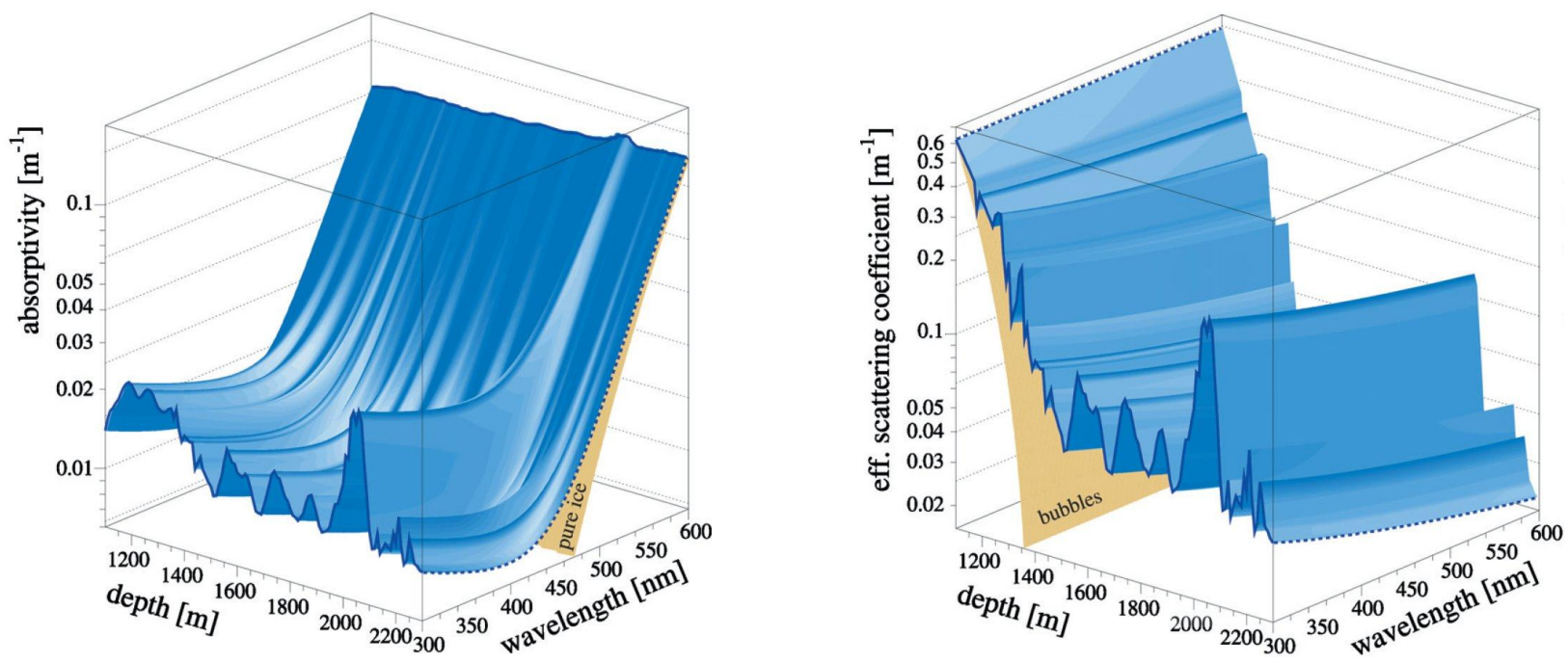

Figure 20. Absorption coefficient (left) and scattering coefficient (right) in the South Polar ice as functions of depth and wavelength.

In Fig. 20, absorption and scattering coefficients are shown as functions of depth and wavelength [106]. The variations with depth are due to bubbles at shallow depth leading to very strong scattering and, at larger depths, to dust and other material transported to Antarctica during varying climate epochs. The quality of the ice improves substantially below a major dust layer at a depth of about $2000-2100 \mathrm{~m}$, with a scattering length about twice as large as for the region above $2000 \mathrm{~m}$. The depth dependence of the optical properties complicates the analysis of the experimental data. Furthermore, the large delays in photon propagation due to the strong scattering cause a worse angular resolution of deep-ice detectors compared to water (see below). On the other hand, the large absorption length, with a cut-off below $300 \mathrm{~nm}$ instead of $350-400 \mathrm{~nm}$ in water, results in better photon collection.

The relatively short distance between optical modules and surface electronics allowed for transporting the analogue signals of the photomultipliers to surface over $2 \mathrm{~km}$ of cable instead of digitising them in situ. This requires a large output signal of the photomultiplier, 
a specification met by the 8-inch R5912-2 from Hamamatsu with 14 dynodes and a gain of $10^{9}$. The first ten strings used copper cables for both high-voltage supply and signal transmission; for the last 9 strings the anode signal was used to drive a LED and the light signal was transmitted to surface via optical fibre. The time resolution achieved was 5-7 ns; given the strong smearing of photon arrival times due to light scattering in ice, this jitter appeared to be acceptable. An event was defined by a majority trigger formed in the surface counting house, requesting $\geq 8$ hits within a sliding window of $2 \mu \mathrm{s}$.

Time calibration of the AMANDA array was performed with a YAG laser at surface, sending short pulses via optical fibres to each optical module. This laser system was also used to measure the delay of optical pulses propagating between strings and so to determine the optical ice properties as well as the inter-string distances. A nitrogen laser $(337 \mathrm{~nm})$, halogen lamps (350 and $380 \mathrm{~nm}$ ) and LED beacons $(450 \mathrm{~nm}$ ) located in deep ice yielded further information about the ice properties across a large range of wavelengths. The measured time delays were fitted and the resulting parameterisations implemented in the probability density functions for the reconstruction procedure (see Sect. 3.6).

A big advantage compared to underwater detectors is the small photomultiplier noise rate, about $1 \mathrm{kHz}$ in an 8-inch tube, compared to $20-40 \mathrm{kHz}$ due to $\mathrm{K}^{40}$ decays and bioluminescence in lakes and oceans. The contamination of hit patterns from particle interactions with noise hits is thus small and makes hit selection much easier than in water.

The angular resolution of AMANDA for muon tracks is $2^{\circ}-2.5^{\circ}$, with a lower energy threshold around $50 \mathrm{GeV}$. Although better than for Lake Baikal $\left(3^{\circ}-4^{\circ}\right)$, it is much worse than for ANTARES $\left(<0.5^{\circ}\right.$, see below). This is the result of the strong light scattering which deteriorates the original information contained in the Cherenkov cone. The effect is even worse for cascades, where the angular resolution achieved with present algorithms is only $25^{\circ}$ (compared to $5^{\circ}-8^{\circ}$ in water [128]).

\subsection{ANTARES}

Starting with the decline of the DUMAND project (see Sect. 4.1), the participating European groups started to explore options for a deep-see neutrino telescope in the Mediterranean Sea. The NESTOR (see Sect. 4.6), ANTARES and NEMO (see Sect. 4.5) projects were initiated, in the temporal order indicated. The NESTOR and NEMO Collaborations have performed technical R\&D work and deployed prototype installations; they are now, together with the ANTARES collaboration, pursuing further work in the KM3NeT framework (see Sect. 5.2). ANTARES has succeeded in installing and is operating the first working deep-sea neutrino telescope, thus providing the proof of feasibility for such devices.

The ANTARES (Astronomy with a Neutrino Telescope and Abyss environmental RESearch) [144 proposal [114] was presented in 1999. It was based on the operation of a demonstrator string [145, 146] as well as on the results of extensive site exploration campaigns in the region off Toulon at the French Mediterranean coast, indicating that the optical background [147] as well as sedimentation and biofouling [148] are acceptable at that site. The ANTARES design encompasses 12 strings, each carrying 25 "storeys" equipped with three optical modules, an electronics container and calibration devices where necessary. A further string, the "instrumentation line", carries devices for environmental monitoring. The 
strings are anchored to the sea floor with a dead weight and kept upright by a submerged buoy at their top. The cable connecting the storeys encompasses copper leads and optical fibres and at the same time has to sustain the mechanical tension; this combination was found to be conceptually challenging. At the sea floor, electro-optical cables connect the strings to a junction box, from which the main cable goes to shore. The strings are deployed from the surface and subsequently connected to the junction box employing a submersible. The inter-string distances are about $70 \mathrm{~m}$, the vertical distance between adjacent storeys is $14.5 \mathrm{~m}$. The depth at the ANTARES site is $2475 \mathrm{~m}$. The schematic setup is shown in Fig. 21, a detailed technical description can be found in [149].

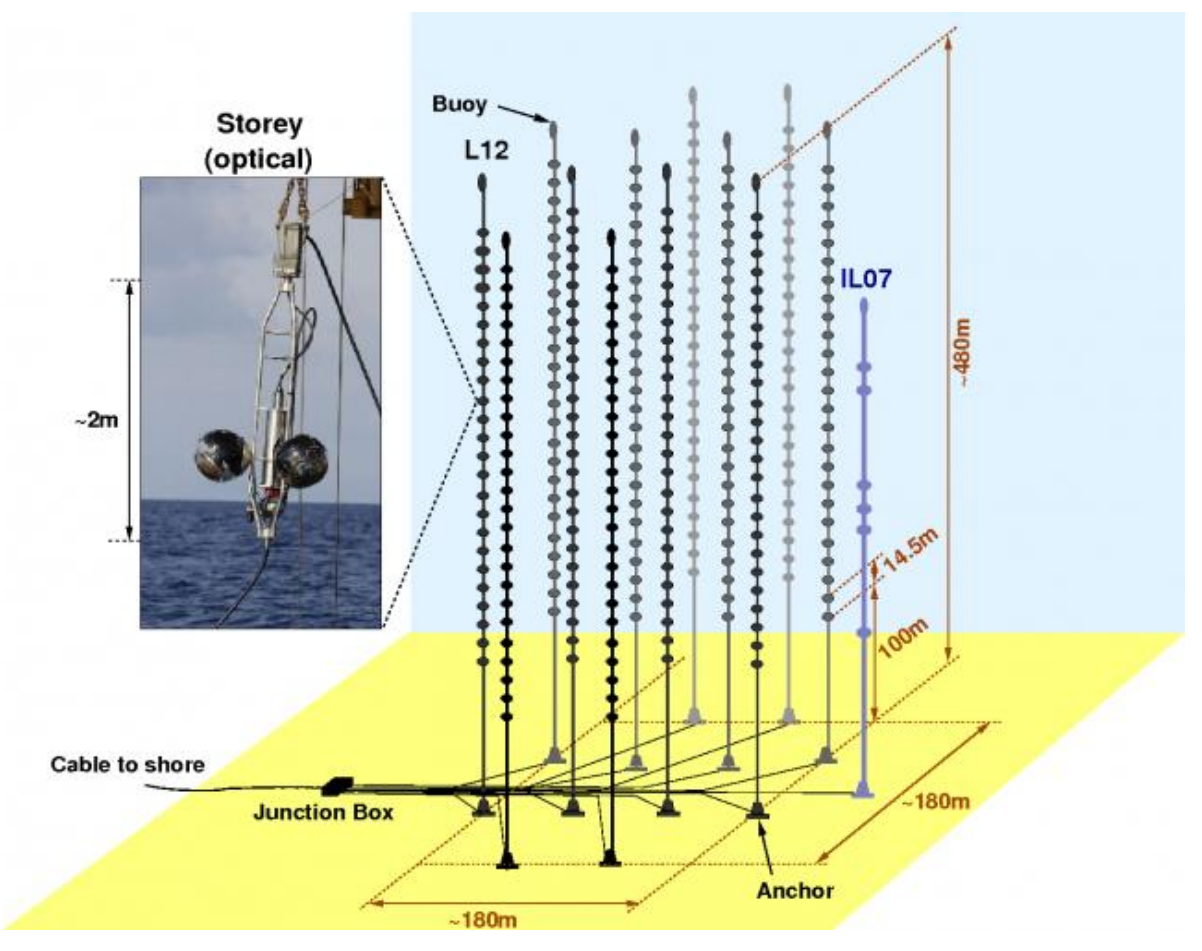

Figure 21. Schematic of the ANTARES detector. Indicated are the 12 strings and the instrumentation line in its 2007 configuration (IL07). Shown as an inset is the photograph of a storey carrying 3 photomultipliers.

The ANTARES construction started in 2002 with the deployment of the main cable and the junction box. In 2002/2003, a preproduction string was deployed and operated for some months. Several technical problems were identified that required further studies, design modifications and the operation of a mechanical test string [150]. The detector in its final configuration was eventually installed in 2006-2008 and has been operational since then, with a break of a few months in 2009 due to a failure of the main cable that required repair.

The ANTARES optical module [151] consists of a 17-inch glass sphere housing a hemispherical 10-inch photomultiplier (Hamamatsu R7081-20), connected to the glass surface by optical gel, and its high-voltage base. A mu-metal cage shields the photomultiplier against the Earth magnetic field (see Fig. 22). A single cable with copper leads is used for voltage supply and for transporting the analogue photomultiplier signals to the digitisation electronics in the electronics container of the storey; dispersion effects are negligible due to the short cable length of about a metre. The optical modules are oriented such that the photomultipliers look downward at an angle of $45^{\circ}$. 


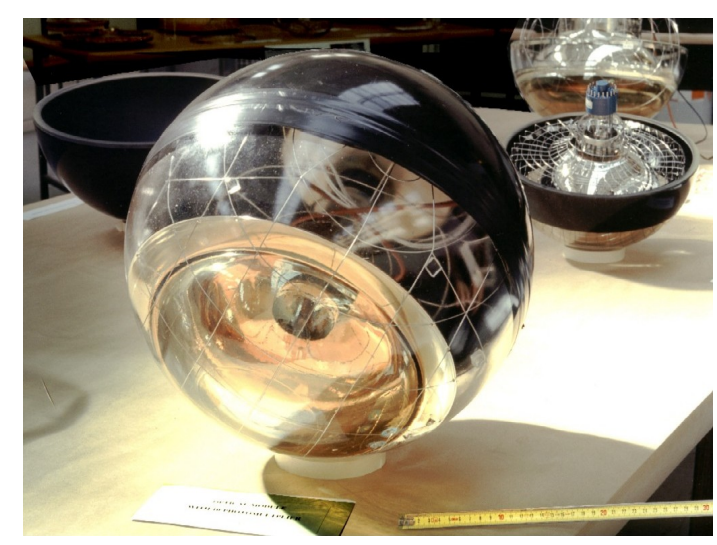

Figure 22. Photograph of an ANTARES optical module. Its upper hemisphere is painted black to reduce background light.

The photomultipliers provide an intrinsic timing resolution (transit time spread) of about $\sigma_{\mathrm{TTS}}=1.3 \mathrm{~ns}$ [152], thus allowing for precise measurements of the arrival times of Cherenkov photons. Owing also to the minute light scattering in deep-sea water [153] and accurate timing and optical module position calibration (see below), muon trajectories can be reconstructed with a precision of about $0.2^{\circ}$ for muon energies exceeding $1 \mathrm{TeV}$ [154]. Degradations are expected for down-going muons (where the direct Cherenkov light is partly outside the photomultiplier acceptance and the fraction of scattered light is therefore increased) and due to background light from $\mathrm{K}^{40}$ decays and bioluminescence. The single photon rate from $\mathrm{K}^{40}$ decays is about $30 \mathrm{kHz}$ per photomultiplier, the bioluminescence rate exhibits a slowly varying baseline of typically a few $10 \mathrm{kHz}$ to a few $100 \mathrm{kHz}$, and second-scale "bursts" in the $\mathrm{MHz}$ region. Efficient data taking is possible up to baseline rates of $200 \mathrm{kHz}$.

The ANTARES data acquisition [155] follows the "all-data-to-shore" concept, i.e. all photomultiplier signals exceeding an adjustable threshold (default 0.3 photo-electrons) are read out, thus avoiding any inter-storey or inter-string trigger processing off-shore. The signals are digitised and time-stamped by the storey electronics and then sent to shore via optical fibres. The resulting data rate is dominated by background hits and exceeds by far the capacity of data mass storage. Data filter software, running on an on-shore farm of PCs, selects event candidates based on multi-hit coincidences and/or multi-photo-electron hits in single photomultipliers. The selected data are stored for offline analysis.

The two main calibration tasks are the synchronisation of the signal time measurements at the individual photomultipliers and the position and orientation monitoring of the optical modules that move with their strings in the sea current. For the timing calibration [156], light signal running times through the optical fibres from shore to each storey and back are used, as well as pulsed light emissions by laser and LED beacons [157]. An accuracy of about $0.5 \mathrm{~ns}$ is achieved, complying with the requirements. The position and orientation calibration [158, 159] uses data from compasses and tiltmeters on each storey and from an acoustic system measuring running times of acoustic pulses between transmitters on the sea floor and receivers (hydrophones) on 5 storeys per string. Also here, the precision of roughly $10 \mathrm{~cm}$, translating into a timing uncertainty of $0.5 \mathrm{~ns}$, is within specifications.

ANTARES is equipped with an acoustic detection system, AMADEUS [160], for feasibility studies towards acoustic neutrino detection. For a more detailed discussion see Sect. 7.3 . 


\subsection{NEMO}

The NEMO (NEutrino Mediterranean Observatory) project [161] is pursued by Italian groups and started 1998 with the objective to investigate the feasibility of a cubic-kilometrescale deep-sea detector and to identify and explore a suitable site.

Technical solutions have been devised, investigated and optimised for an easy-to-deploy, stable and cost-effective detector [162 164. The supposedly most important new concept developed in NEMO is that of "flexible towers" constructed of horizontal bars of a length of up to $15 \mathrm{~m}$, interconnected by ropes forming a tetrahedral structure, so that adjacent bars are positioned orthogonal to each other (see Fig. 23). The optical modules are fixed to the bars. This design has several advantages, in particular (i) that a tower can be folded together and deployed to the sea floor as a compact object that is subsequently unfurled; (ii) the separation of the mechanical tension (carried by the ropes) from the electro-optical backbone cable; (iii) the provision of a 3-dimensional arrangement of photomultipliers per tower, allowing for local reconstruction of muon directions.

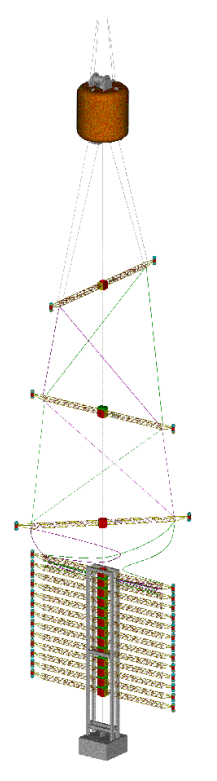

Figure 23. Sketch of the principle of a flexible tower during the unfurling process. Note that exact configuration and the packaging of the bars does not correspond to the current design framework.

Concurrently with the technical developments, a suitable site at a depth of $3.5 \mathrm{~km}$, about $100 \mathrm{~km}$ off Capo Passero at the South-Eastern coast of Sicily has been identified and investigated during various campaigns (see [103] and references therein).

During the first prototyping phase ("NEMO Phase-1"), a cable to a test site near Catania at a depth of $2 \mathrm{~km}$ was installed and equipped with a junction box. In 2007, a "minitower" with 4 bars was deployed, connected and operated for several weeks. Although the data taking period was limited to a few months due to technical problems, the mini-tower provided the proof of concept for the technologies and most of the components employed. The flux of atmospheric muons was determined in good agreement with the expectations from simulation [165].

The Phase-2 [166] setup includes shore infrastructure at Capo Passero and a $100 \mathrm{~km}$ long cable to the site at $3.5 \mathrm{~km}$ depth; both are meanwhile in place. A remotely operated vehicle 
(ROV) is available for the deep-sea operations. A mechanical test tower of limited size was successfully deployed and unfurled in early 2010. The plans to deploy a full-size prototype tower will be pursued in the KM3NeT framework.

During the Phase-1 activities, an acoustic setup, $\mathrm{O} \nu \mathrm{DE}$ (Ocean noise Detection Experiment), was operated for almost two years and yielded important data on the acoustic deep-sea background [167] and also on sperm whales [168].

\subsection{NESTOR}

NESTOR [169] started out as a Greece-centred, originally international collaboration around the beginning of the 1990s. A series of workshops was held in 1991-1993 at the homonymous institute in Pylos at the West coast of the Peloponnesus and a detector concept presented in 1994 [170]. The basic idea is to attach the optical modules to rigid hexagonal structures ("floors") of $30 \mathrm{~m}$ diameter, of which 12 are stacked to a tower with 20-30 m interfloor distance. The deployment would be from the sea top, the connection to the main cable or already-deployed components being made in the sequence recovery-connectionredeployment, thus avoiding the use of deep-sea submersibles.

Several suitable site options off the coast of Pylos have been identified. They are between $3000 \mathrm{~m}$ and $5200 \mathrm{~m}$ deep and have distances to shore of $30-50 \mathrm{~km}$. The NESTOR collaboration has performed numerous studies on the site characteristics, most recently on the water optical properties (see [103] and references therein).

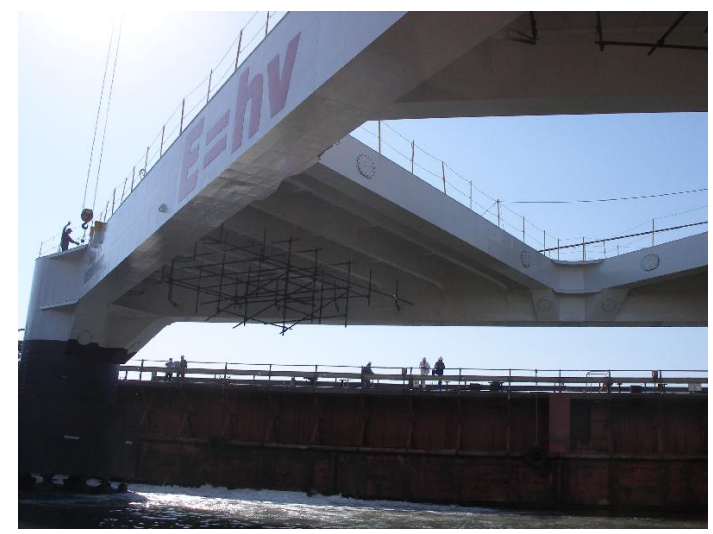

Figure 24. Photograph of the Delta Berenike platform in the final phase of its construction.

After a long phase of technical development a cable was installed to a site at $4 \mathrm{~km}$ depth. A single, reduced-size floor with 12 optical modules was deployed, connected and operated for more than a month; its operation had to be terminated due to a failure of the cable to shore. The data recorded sufficed to validate the detector functionality within specifications [171] and to measure the atmospheric muon flux, which was found to agree with the expectations [172].

To facilitate the deployment of the floors, NESTOR has developed and constructed a triangular working platform for sea operations, the Delta Berenike (see Fig. 24). The platform with side lengths of about $50 \mathrm{~m}$ is carried by three cylindrical pontoons at its apices and has a triangular aperture for deployment operations. 


\section{Second-Generation Neutrino Telescope Projects}

There are three projects which reach, or even exceed, the size originally conceived by the DUMAND pioneers: IceCube at the South Pole, in its basic configuration, has been completed in December 2010; KM3NeT ( $\mathrm{km}^{3}$ Neutrino Telescope) in the Mediterranean Sea and GVD (Gigaton Volume Detector) in Lake Baikal are in their preparatory/prototyping phase. IceCube instruments $1 \mathrm{~km}^{3}$ of ice. GVD is also planned to cover $1 \mathrm{~km}^{3}$, but with a much higher energy threshold than IceCube. KM3NeT envisages an instrumented volume of several $\mathrm{km}^{3}$. KM3NeT and GVD could be completed by 2017 but will start data taking earlier with intermediate configurations. It is expected that these detectors will form a global network or even join to a global neutrino observatory.

\subsection{IceCube}

IceCube [173, 174] is the successor of AMANDA. It consists of 5160 digital optical modules (DOMs) installed on 86 strings at depths of 1450 to $2450 \mathrm{~m}$ in the Antarctic ice [175]. 320 further DOMs are installed in IceTop, an array of detector stations on the ice surface directly above the strings (see Fig. 25). AMANDA, initially running as a low-energy subdetector of IceCube, was decommissioned in 2008 and replaced by DeepCore, a high-density sub-array of six strings at large depths (i.e. in the best ice layer) at the centre of IceCube.

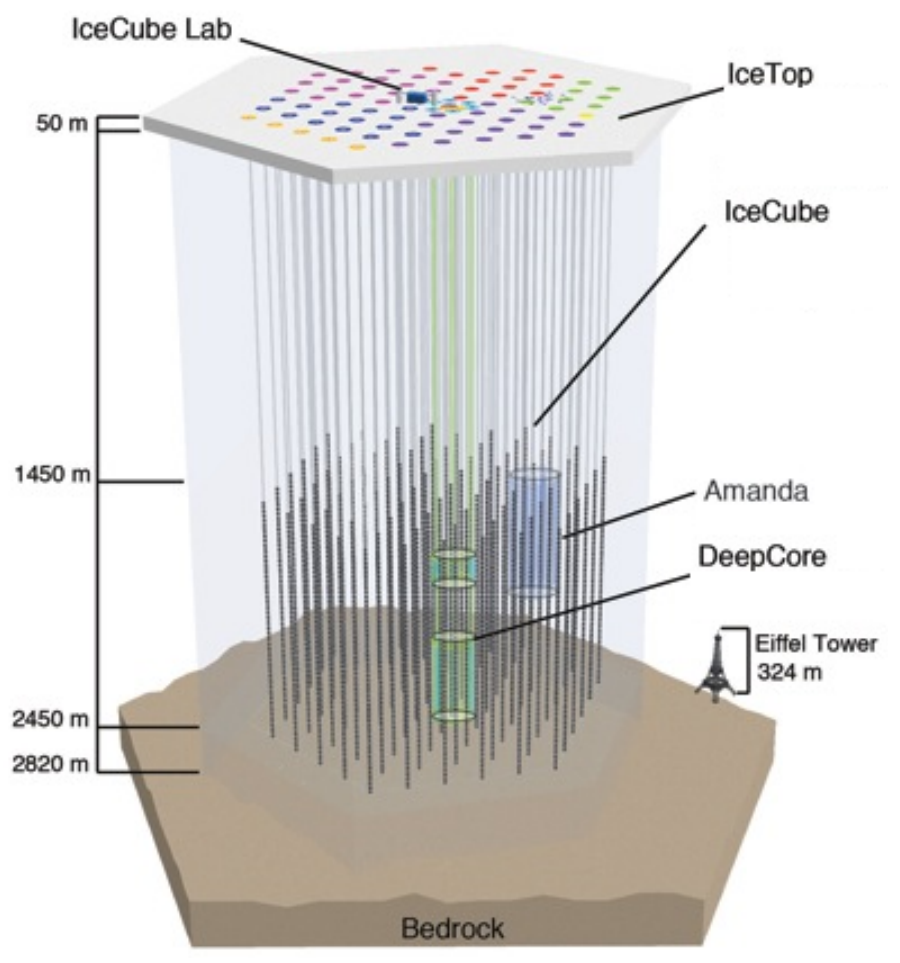

Figure 25. Schematic view of the IceCube neutrino observatory. Also shown is the location of AMANDA (cf. Sect. 4.3) and of DeepCore, a nested low-threshold array (cf. Sect. 5.1.1). At the surface, the air shower array IceTop (cf. Sect. 5.1.2) and the IceCube counting house are indicated. The Eiffel tower is shown to scale for a size comparison.

For IceCube construction, the thermal power of the hot-water drill factory has been upgraded to $5 \mathrm{MW}$, compared to $2 \mathrm{MW}$ for AMANDA, thus reducing the average time to drill a $2450 \mathrm{~m}$ deep hole with a diameter of $60 \mathrm{~cm}$ to 35 hours. The subsequent installation 
of a string required typically 12 hours. Installation of IceCube started in January 2005 with the first string and was completed with the deployment of the $86^{\text {th }}$ string at Dec. 18, 2010.

As the components are not accessible after refreezing of the holes, the IceCube architecture avoids single point failures in the ice. A string carries 60 DOMs, with 30 twisted copper pair cables providing power and communication. Neighbouring DOMs share the same wire pair and are thus connected to enable fast local coincidence triggering in the ice.

A schematic view of a DOM is shown in Fig. 26. A 10-inch photomultiplier (Hamamatsu R7081-02) is embedded in a 13-inch glass sphere. A mu-metal grid reduces the influence of the Earth magnetic field. The programmable high voltage is generated inside the DOM. The average photomultiplier gain is set to $10^{7}$. Signals are sent to the main board where they are digitised by a fast analogue transient waveform recorder (ATWD, $3.3 \mathrm{~ns}$ sampling) and by a FADC ( $25 \mathrm{~ns}$ sampling). The photomultiplier signal is amplified by 3 different gains to extend the dynamic range of the ATWD to 16 bits. The resulting linear dynamic range is 400 photo-electrons in $15 \mathrm{~ns}$; the dynamic range integrated over $2 \mu \mathrm{s}$ is about 5000 photoelectrons [176]. The digital section of the main board is based on a field-programmable gate array (FPGA), which communicates with the surface electronics and allows for uploading new program code. LEDs on a "flasher board" emit calibration pulses at a wavelength of $405 \mathrm{~nm}$ which can be adjusted in intensity over a wide range up to $10^{11}$ photons.

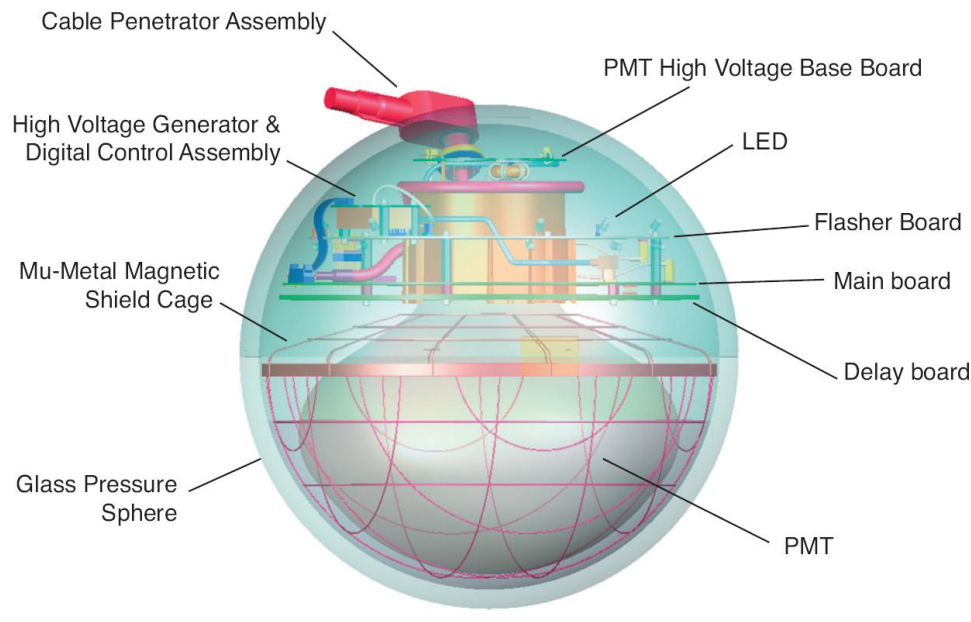

Figure 26. Schematic view of an IceCube Digital Optical Module (DOM). The analogue photomultiplier signals are digitised on the main board. For details see [176].

All digitised photomultiplier pulses are sent to the surface. In order to compress data for isolated hits which are mostly noise pulses, the full waveform is only sent for pulses appearing in local (neighbour or next-to-neighbour) coincidences on a string. All DOMs have precise quartz oscillators providing local clock signals, which are synchronised every few seconds to a central GPS clock. The time resolution is about $2 \mathrm{~ns}$. The noise rate for DOMs in the deep ice is about $540 \mathrm{~Hz}$ and is reduced to ca. $280 \mathrm{~Hz}$ if an artificial deadtime of $50 \mu \mathrm{s}$ is applied to discard after-pulses (only for the supernova burst trigger, see Sect. 5.1.3). These very low noise rates are essential for the detection of the low-energy neutrino emission associated with a supernova collapse (see Sect. 5.1.3.

At the surface, 8 custom PCI cards per string provide power, communication and time calibration. Subsequent processors sort and buffer hits until the array trigger and event 
builder process is completed. This architecture allows for a deadtime-free operation. The raw data rate of the full array is about $800 \mathrm{~GB} /$ day, which are written to tape and processed online on a computer farm to extract interesting event classes, like up-going muon candidates, high-energy events, IceTop/IceCube coincidences, cascade events, events from the direction of the Moon or events in coincidence with Gamma Ray Bursts (GRBs). The filtered data stream (about $70 \mathrm{~GB} /$ day) is transmitted to the Northern hemisphere via satellite.

The muon angular resolution achieved with present reconstruction algorithms is about $1^{\circ}$ for $1 \mathrm{TeV}$ muons. It is expected that improved reconstruction algorithms using the full waveform will lead to a resolution below $0.5^{\circ}$ for energies above $10 \mathrm{TeV}$. The very clear ice below a depth of $2100 \mathrm{~m}$ has a particular potential for improved resolution. This will be even more important for the angular reconstruction of cascades, for which the presently achieved angular resolution is only $30^{\circ}$ - much worse than for water, mainly due to the strong light scattering in ice.

\subsubsection{DeepCore}

The geometry of DeepCore is sketched in Fig. 27. DeepCore consists of 7 central standard IceCube strings plus additional 6 special strings. Ten of the DOMs of these strings are arranged at $1750-1850 \mathrm{~m}$ depth, above the dust layer with bad optical transparency. They are used as veto-detector for the deeper component. The deep component comprises 50 DOMs per string and is installed in the very clear ice at depths between 2100 and $2450 \mathrm{~m}$, where the effective scattering length ranges up to $50 \mathrm{~m}$ and the absorption length to $230 \mathrm{~m}$. The six additional DeepCore strings are equipped with photomultipliers with an enhanced quantum efficiency (Hamamatsu R7081-MOD).

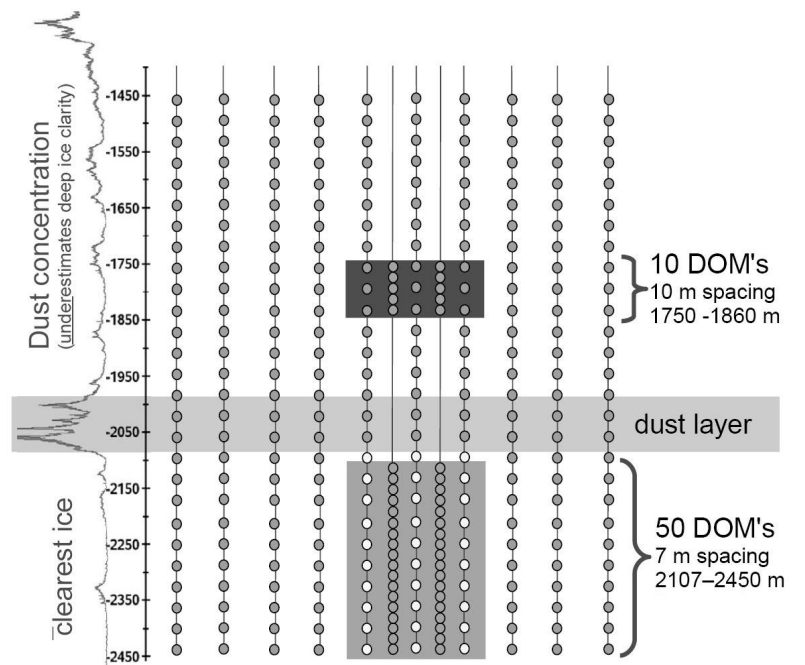

Figure 27. Layout of the DeepCore sub-detector. Shown are the positions of the DeepCore DOMs; for a 3-dimensional representation see Fig. 25. The depth profile of the ice transparency is indicated on the left.

DeepCore has a factor of about six better sensitivity in photon collection than IceCube, due to the smaller spacing between strings (72 instead of $125 \mathrm{~m}$ ) and OMs along a string (7 instead of $17 \mathrm{~m}$ ); the better ice quality; and the $30 \%$ higher quantum efficiency of the new photomultipliers. Together with the veto provided by IceCube, this results in an expected threshold of less than $10 \mathrm{GeV}$. This opens a new window for oscillation physics not tested 
by other experiments and allows for probing dark matter models not covered by direct searches. The veto will also allow for identification of neutrinos from above if they interact within DeepCore. This enlarges the field of view of IceCube to the full sky [177].

\subsubsection{IceTop}

IceCube is the only large neutrino telescope which can be permanently operated together with a surface air shower array, IceTop [178]. IceTop consists of tanks filled with ice, each instrumented with 2 DOMs. With IceTop, the energy spectrum of air showers can be measured up to primary particle energies around $10^{18} \mathrm{eV}$. The combination of IceTop information (reflecting dominantly the electron component of the air shower) and IceCube information (muons from the hadronic component) restricts the mass range of the primary particle. The comparison of air shower directions measured with IceTop and directions of muons from these showers in IceCube establishes a tool for angular calibration of IceCube (absolute pointing and angular resolution).

\subsubsection{IceCube as a supernova burst detector}

Last but not least, IceCube can be operated in a mode that is only possible in ice: The detection of burst neutrinos from supernovae. The low dark-count rate of the photomultipliers $(280 \mathrm{~Hz}$, see above) allows for detection of the feeble increase of the summed count rates of all photomultipliers during several seconds, which would be produced by millions of interactions of few-MeV neutrinos from a supernova burst [179, 180. IceCube records the counting rate of all photomultipliers in millisecond steps. A supernova in the centre of the Galaxy would be detected with extremely high significance and the onset of the pulse could be measured in unprecedented detail. Even a SN 1987A-type supernova in the Large Magellanic Cloud would provide a recognisable signal and be sufficient to provide a trigger to the SuperNova Early Warning System, SNEWS [181].

\section{2. $K M 3 N e T$}

In recognition of the fact that at least a cubic-kilometre sized detector will be necessary to really observe abundant astrophysical high-energy neutrino sources, the High Energy Neutrino Astronomy Panel (HENAP) of the PaNAGIC ${ }^{2}$ Committee of IUPAP $^{3}$ has concluded in its 2002 report [182] that "a km³-scale detector in the Northern hemisphere should be built to complement the IceCube detector being constructed at the South Pole".

Following this recommendation, the Mediterranean neutrino telescopes groups - together with deep-sea technology and marine science groups - have formed the KM3NeT collaboration to prepare, construct and operate such a device. In 2006-2009, a Design Study, supported with $9 \mathrm{M} €$ by the EU, was conducted; its major achievements are a Conceptual Design Report (CDR) [183] and a Technical Design Report (TDR) [103. A further EU project, the KM3NeT Preparatory Phase (2008-2012) provides resources and a framework for work directed towards solving the funding, governance, legal and strategic questions and

\footnotetext{
${ }^{2}$ Particle and Nuclear Astrophysics and Gravitation International Committee

${ }^{3}$ International Union of Pure and Applied Physics
} 
also for engineering activities. KM3NeT will be a deep-sea research infrastructure hosting a neutrino telescope, but also providing continuous, long-tern access to deep-sea measurements to a variety of science communities, such as marine biologists, oceanographers, geophysicists and environmental scientists. In recognition of its high scientific potential, KM3NeT has been included in the priority project list of the European Strategy Forum on Research Infrastructures, ESFRI [184, 185].

Based on the experience and expertise of the first-generation projects, a variety of new, cost-effective design solutions for the neutrino telescope have been elaborated. The original goal of reducing the capital investment for a cubic kilometre of instrumented sea water to $200 \mathrm{M} €$ has been outperformed by a factor of at least 4 . Whereas the TDR still presented a set of design options, in particular for the mechanical structure of the strings/towers ("detection units (DUs)") and the optical modules, further investigations have meanwhile led to convergence on a specific design, which will be described in the following. Since final prototyping and deployment tests are still to come, the discarded solutions partly serve as backup options.

For the mechanical DU structure, a solution along the NEMO design (see Sect. 4.5) has been chosen. The bars will be $6 \mathrm{~m}$ long and be equipped with one optical module (see below) at each end; a schematic view is given in Fig. 28. Oil-filled hoses in equipressure with the deep-sea water will be used as vertical cables for electrical leads and optical fibres. Two such backbone cables, wound loosely around the ropes, will be used per DU, with break-outs for two copper leads and one optical fibre at each optical module. The DUs will be stacked in a cubicle pile for transport and deployment, fitting into a standard shipping container. The deployment will proceed as discussed in Sect. 4.5.
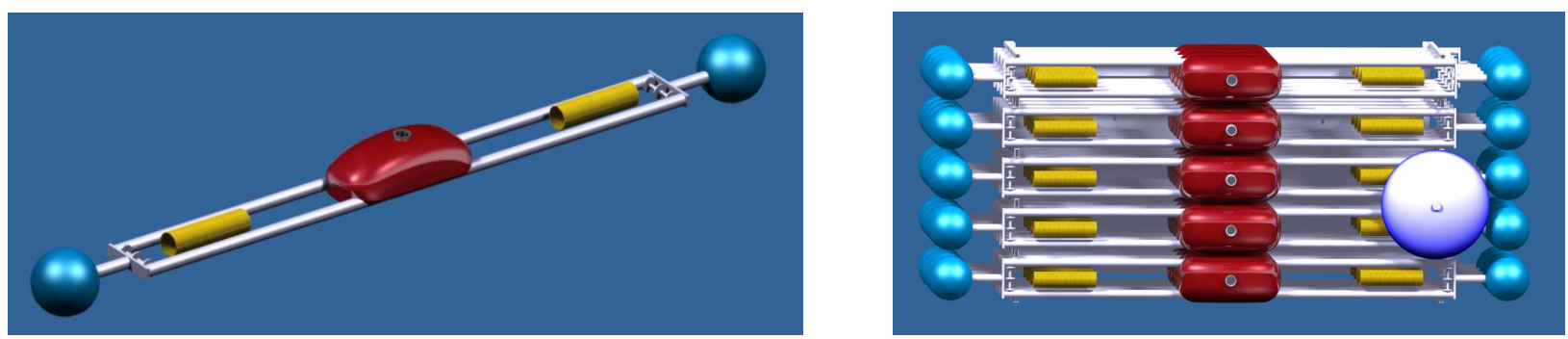

Figure 28. Left: Schematic drawing of a bar of the KM3NeT DU. The overall length is slightly below $6 \mathrm{~m}$. The red object in the centre is a buoy made of syntactic foam, the yellow cylinders house the ropes and vertical backbone cables before unfurling. Right: A full DU stacked for transport and deployment (top view). The round bluish object is the top buoy.

The digital optical module (DOM, Fig. 29) will be a 17-inch glass sphere, equipped with 31 3-inch photomultipliers, their high-voltage bases and the digitisation electronics. Highvoltage bases with a power consumption as low as $140 \mathrm{~mW}$ for a complete optical module have been designed for this application. The photomultipliers are oriented from vertically downwards to about $45^{\circ}$ upwards. They are supported by a foam structure and fixed to the glass sphere by optical gel. An aluminium structure is used to conduct the heat to the glass and to provide support for the electronic boards. Major advantages of this design are: 

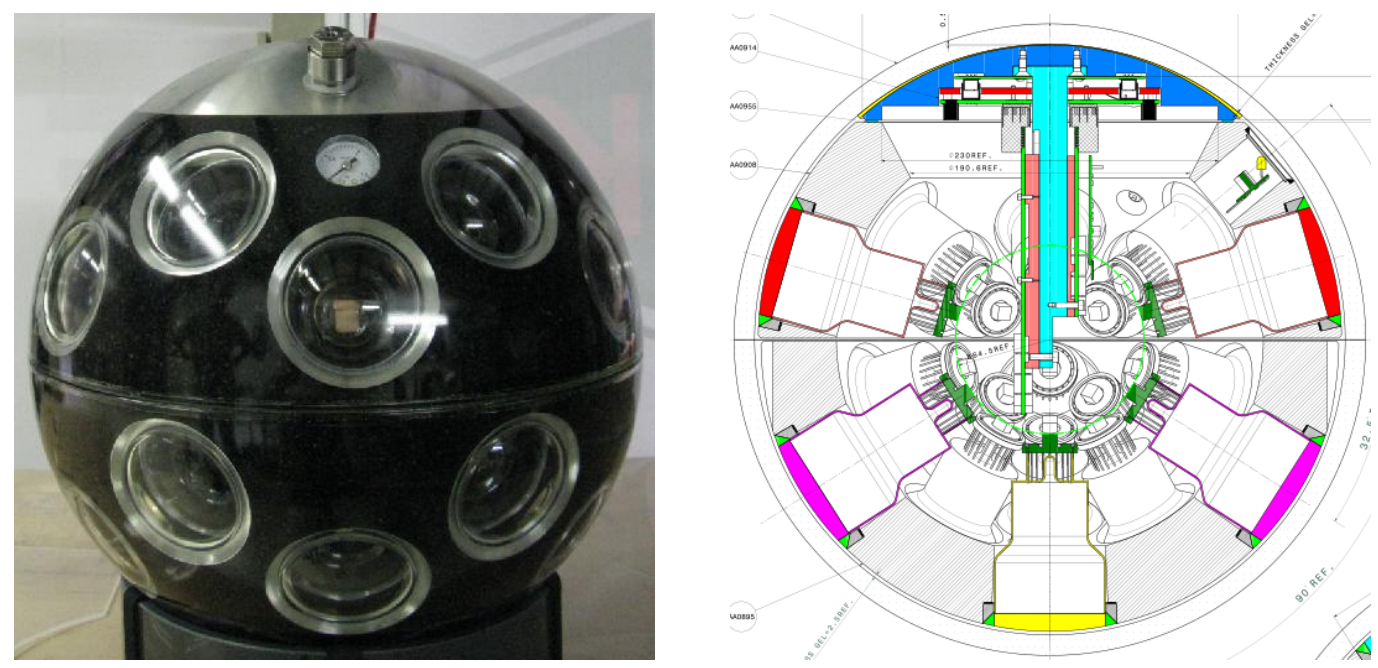

Figure 29. The KM3NeT digital optical module (DOM). Left: Photograph of a prototype; right: technical drawing, with the mushroom-shape heat conductor and the electronics components.

- The overall photocathode area exceeds that of a 10-inch photomultiplier by more than a factor of three; a further increase is possible by extending the light collection area using reflective rings [186]. The number of penetrators and separate electronics containers, which are expensive and failure-prone, is thus reduced to a minimum.

- Since the photomultipliers are read out individually, a very good one-vs.-two photoelectron resolution is obtained, which is essential for efficient online data filtering.

- Some directional sensitivity is provided.

The KM3NeT data acquisition will follow the all-data-to-shore concept (see Sect. 4.4). For each photomultiplier, the time intervals during which the analogue output signal exceeds an adjustable threshold will be digitised and sent to shore. This time-over-threshold information allows for precise photo-electron counting for small signals and provides a logarithmic measure of the amplitude for large signals, i.e. effectively an infinite dynamic range. The technical implementation can either be achieved using a custom-designed ASIC [103] or a fast time-to-digital converter (TDC) on a field programmable gate array (FPGA). The latter option is new and might significantly simplify the system. The digitised data are transported to shore via optical point-to-point connections using the Gigabit ethernet protocol and dense wavelength division multiplexing. The system is driven by on-shore lasers, which allows for easy maintenance and also for sending clock signals to the DOMs by the same laser beam that carries the photomultiplier signals on its way back to shore.

Time and position calibration will be based on the same principles as in ANTARES (see Sect. 4.4). For the acoustic receivers on the DUs, the stand-alone hydrophones (that require extra penetrators) will presumably be replaced by piezo elements glued to the inner glass surface of the DOMs.

The full KM3NeT neutrino telescope will consist of about 300 DUs. Their geometrical layout on the sea-floor (the "footprint") is still subject to optimisation. For the sensitivity 
studies in the TDR two equal-size homogeneous blocks of hexagonal layout were assumed, with an inter-DU distance of $180 \mathrm{~m}$ and a vertical distance between adjacent bars of $40 \mathrm{~m}$. The partition into two blocks takes into account the facts that deployment and maintenance of the deep-sea cable network becomes increasingly difficult for large homogeneous setups, and that at least two main cables to shore will be needed for the full detector.

Since the exact footprint is not yet known, the deep-sea cable network topology is still open. It will either be star-like with a primary and a set of secondary junction boxes or consist of a cable ring surrounding the detector, with several primary junction boxes connected directly to the DUs. In the cable network, single points of failure for the full or at least substantial parts of the detector are unavoidable, making recovery and maintenance operations necessary. In contrast, no maintenance is planned for the DUs themselves.

The time-line of the further steps towards construction and operation of KM3NeT is shown in Fig. 30. The three sites of ANTARES, NEMO and NESTOR are under consideration for KM3NeT. The site question has scientific aspects that have been investigated in detail (depth, water transparency, bioluminescence etc., see [103] and references therein); additionally, the site choice is strongly linked to the availability of regional funding sources and is therefore closely interwoven with a political decision on KM3NeT. It is currently hoped that a site decision can be made by late 2011 or early 2012. Subsequently, the final technical design will be worked out and presented in a detailed proposal. Assuming that funding, legal and administrative issues are sorted out by then, it will be possible to launch production at that point. Data taking will start as soon as the first DUs are operational. From a very early stage of its construction on, the data from the KM3NeT neutrino telescope will exceed data from first-generation Northern-hemisphere neutrino telescopes in quality and statistics and thus provide an exciting discovery potential.

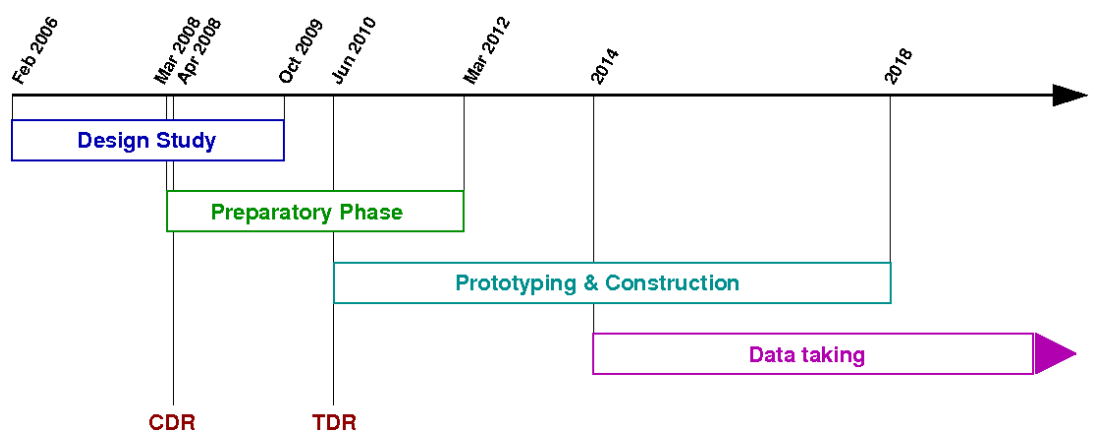

Figure 30. Time-line towards KM3NeT construction and operation.

\subsection{GVD in Lake Baikal}

The Baikal Collaboration plans the stepwise installation of a kilometre-scale array in Lake Baikal, the Gigaton Volume Detector, GVD [187]. It will consist of strings which are grouped in clusters of eight (see Fig. 31). This results in a relatively flexible structure, which allows for rearranging the clusters and meets best the deployment conditions from the ice. Each string carries 24 optical modules spaced uniformly from $900 \mathrm{~m}$ down to about $1250 \mathrm{~m}$ depth [188]. The modules will house 10-inch photomultipliers (likely Hamamatsu R7081-HQE) with a peak quantum efficiency of about $35 \%$. 

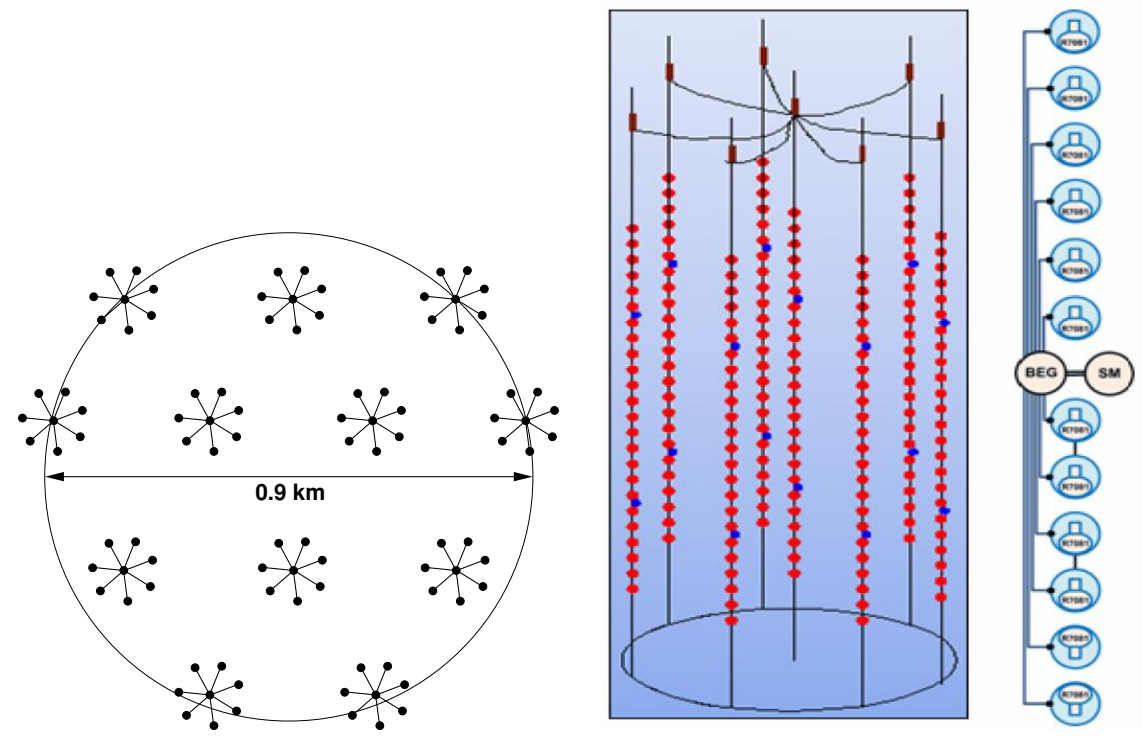

Figure 31. The Baikal Gigaton Volume Detector (GVD). Left:

Arrangement of the 12 clusters; middle: One cluster; right: Schematic view of a string section.

The optical modules on a string are grouped into two sections, each consisting of $12 \mathrm{OMs}$, a service module (SM) and the electronics unit (BEG) with its $200 \mathrm{MHz}$ FADCs (see Fig. 31 right). Analogue signals from the optical modules are transmitted to the BEG through coaxial cables. A trigger is formed by a coincidence of any neighbouring optical modules. Digitised waveforms for each triggered channel are transmitted via Ethernet from the BEG to the central underwater micro-PC of the cluster. The cluster DAQ provides inter-section time synchronisation, on-line data selection, and communication to shore through an optical cable. Prototype strings have been operated in 2009 and 2010. They have demonstrated a time accuracy of about 2 ns. In April 2011, a prototype cluster with three mini-strings and all key elements of DAQ electronics and the communication system was deployed.

Simulations have been performed for 96 strings in 12 clusters and a total of 2304 OMs. A compromise between large volume for cascade detection and reasonable efficiency for muons was found for an instrumented height of $345 \mathrm{~m}$, a cluster diameter of $120 \mathrm{~m}$ and a vertical spacing between optical modules of about $15 \mathrm{~m}$ [188]. At trigger level, the effective detection area for muons with energies above $3 \mathrm{TeV}$ is $0.2-0.5 \mathrm{~km}^{2}$; the effective detection volume for cascades above $50 \mathrm{TeV}$ is $0.3-0.8 \mathrm{~km}^{3}$. We note that cuts for background suppression will reduce these values significantly, in particular at lower energies. The directional accuracies are $0.5-1.0^{\circ}$ for muons and $3-7^{\circ}$ for cascades.

A threshold for muons of about $3-10 \mathrm{TeV}$ appears to be rather high when compared to IceCube and KM3NeT. On the other hand, the optimum energy cut to obtain the best signal-to-noise ratio (extraterrestrial versus atmospheric neutrinos) for the weakest detectable sources is at a few $\mathrm{TeV}$ for point sources, and in the $100 \mathrm{TeV}$ range for diffuse fluxes [189]. Therefore, a sparse detector configuration such as GVD may offer a favourable physics/cost ratio for neutrino signals extending to the $100 \mathrm{TeV}$ region and beyond, as e.g. expected for Gamma Ray Bursts and AGN jets. 


\section{Physics Results and Perspectives}

After two decades of data taking with neutrino telescopes, a lot has been learned despite the fact that not a single high-energy neutrino of cosmic origin has been clearly identified as yet. In this section, the results as of early 2011 are presented for atmospheric neutrinos (Sect.6.1), for cosmic neutrinos including those from supernova bursts (Sect. 6.2), for searches for Dark Matter and other exotic particles (Sect. 6.3) and from cosmic-ray studies with IceCube/IceTop (Sect. 6.4)

\subsection{Atmospheric neutrinos}

Atmospheric neutrinos and muons are produced in cosmic-ray interactions in the atmosphere. Up to energies of about $100 \mathrm{TeV}$, their flux is dominated by pion and kaon decays. The corresponding neutrinos are referred to as "conventional" atmospheric neutrinos. The spectrum follows approximately an $E^{-3.7}$ shape. It can be calculated with uncertainties of about $25 \%$ in the energy range $100 \mathrm{GeV}-1 \mathrm{TeV}$ (see [190] and references therein, a widely used analytical calculation is given in [59]). At higher energies, "prompt" atmospheric neutrinos from the decay of charm and bottom particles take over. These particles decay before having a chance for further interactions, and the resulting neutrinos therefore closely follow the primary cosmic ray power law spectrum, i.e. an $E^{-2.7}$ shape.

An almost background-free separation of neutrino-induced upward-going muons from the huge background of downward-going muons is the central requirement for an underwater or under-ice telescope (see Sect. 3.4.1). The first-generation experiments (Baikal, ANTARES and AMANDA) have quickly mastered this challenge, even more so IceCube. Figure 32 shows the rate of muons as a function of the zenith angle $\theta$ as measured with ANTARES. Below the horizon $(\theta<0)$ the rate is well described by the expectation for atmospheric neutrinos, above the horizon by that for atmospheric muons.

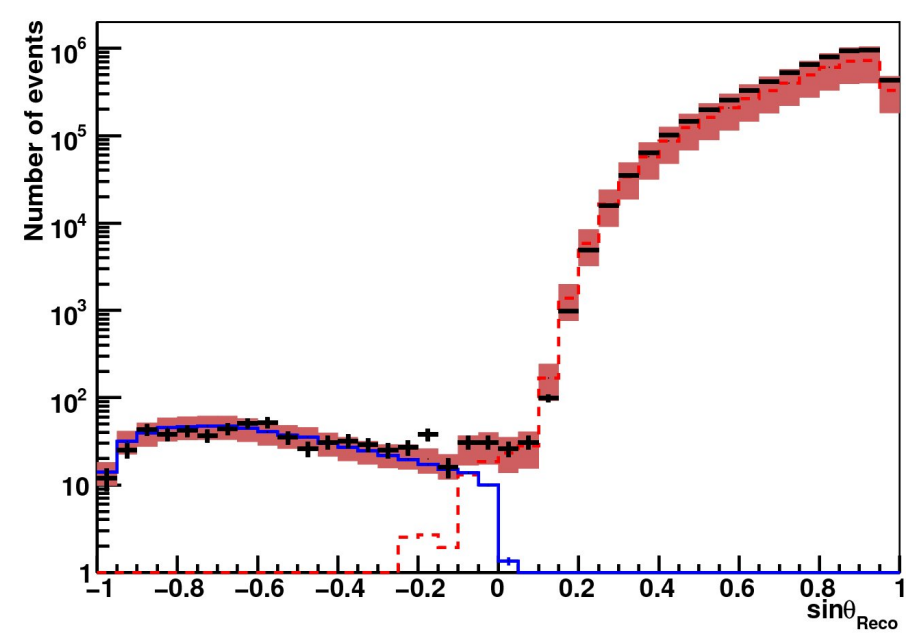

Figure 32. Number of reconstructed muons in the 2008 ANTARES data, as a function of the reconstructed zenith angle $\theta_{\text {Reco }}$ (black error bars). Also indicated are the simulation results for atmospheric muons (red dashed), and muons induced by atmospheric neutrinos (blue). The shaded band indicates the systematic uncertainties. Figure taken from [125].

The energy spectrum of atmospheric neutrinos is inferred from muon neutrino charged current events since they offer the best event statistics and, for this measurement, the least background. Measuring the energy spectrum is difficult since the weak dependence of the 
Cherenkov light yield (i.e. of $\mathrm{d} E_{\mu} / \mathrm{d} x$ ) on $E_{\mu}$ needs to be exploited; additionally, one has to take into account that most muons lose an unknown fraction of their energy before reaching the detector. The neutrino energy spectrum is therefore determined with the help of sophisticated deconvolution procedures, inducing significant point-to-point correlations. Two underground experiments have published atmospheric neutrino energy spectra up to a few TeV: The Fréjus experiment 191 and Super-Kamiokande [192. AMANDA and IceCube have extended this energy range by two orders of magnitude, up to 200 and $400 \mathrm{TeV}$, respectively. Figure 33 shows the spectra as published by these experiments. The data are well compatible with the predictions for conventional atmospheric neutrinos. In particular, no excess at high energies is observed as yet; improved data statistics from IceCube, however, will soon allow to test flux models for prompt neutrinos from the decay of charm and bottom hadrons, which would show up as a shoulder at some $100 \mathrm{TeV}$. In addition, constraints on the neutrino flux in this energy range and beyond are used to place upper limits on the flux of extra-terrestrial sources with a hard spectrum like $E_{\nu}^{-2}$ (see Sect. 6.2).

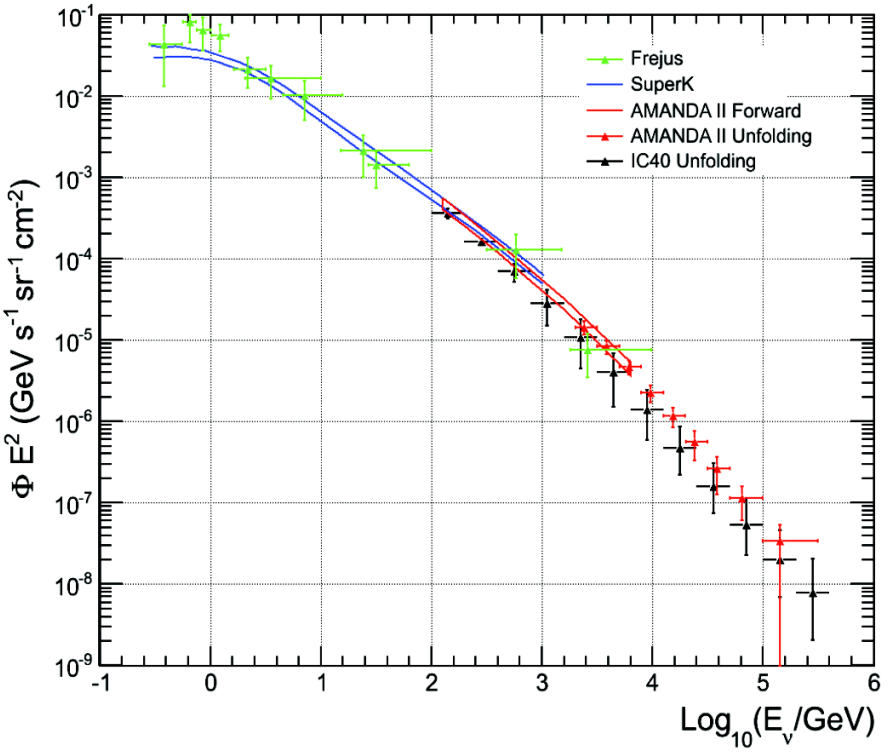

Figure 33. Energy spectrum of atmospheric neutrinos. Green triangles: Fréjus [191; blue band: Super-Kamiokande 192; red band: AMANDA forward folding analysis [98; red triangles: AMANDA unfolding analysis 193]; black triangles: IceCube-40 unfolding analysis [194].

Atmospheric neutrinos also provide a tool to investigate neutrino oscillations. Standard oscillation lengths scale with $E_{\nu}$. For distances of the order of the Earth diameter the first oscillation minimum is at $E_{\nu} \simeq 24 \mathrm{GeV}$ (see Fig. 34). Violation of Lorentz Invariance (VLI), as suggested by certain quantum gravity theories, also leads to oscillation effects, with oscillation lengths that scale with $1 / E_{\nu}$ in the simplest case, $n=1$ (see Sect. 2). The left panel of Fig. 34 shows the survival probability as a function of neutrino energy for a baseline of the Earth diameter and assuming maximal mixing for all three cases (conventional oscillations, VLI oscillations and quantum decoherence, with the assumptions given in the figure caption). Large new neutrino telescopes are an ideal tool to look for non-standard oscillations, due to their ability to collect large statistics of high-energy neutrinos having travelled over distances of several thousand kilometres. From the non-observation of a deficit at high energies and the angular dependence, limits on the relevant parameters can 
be derived. For $n=1$ the $90 \%$ CL upper limits from AMANDA are $2.8 \times 10^{-27}$ for $\Delta \delta$ and $1.2 \times 10^{-27}$ for $D_{i}$ (see Sect. 2). This is similar to limits obtained from the much smaller underground experiments. For $n=2$ the limits are $2.7 \times 10^{-31} \mathrm{GeV}^{-1}$ for $\Delta \delta$ and $1.3 \times 10^{-31} \mathrm{GeV}^{-1}$ for $D_{i}^{*}=D_{i} / E^{n-1}[98$ ]. IceCube promises another factor of ten in sensitivity over the forthcoming years.
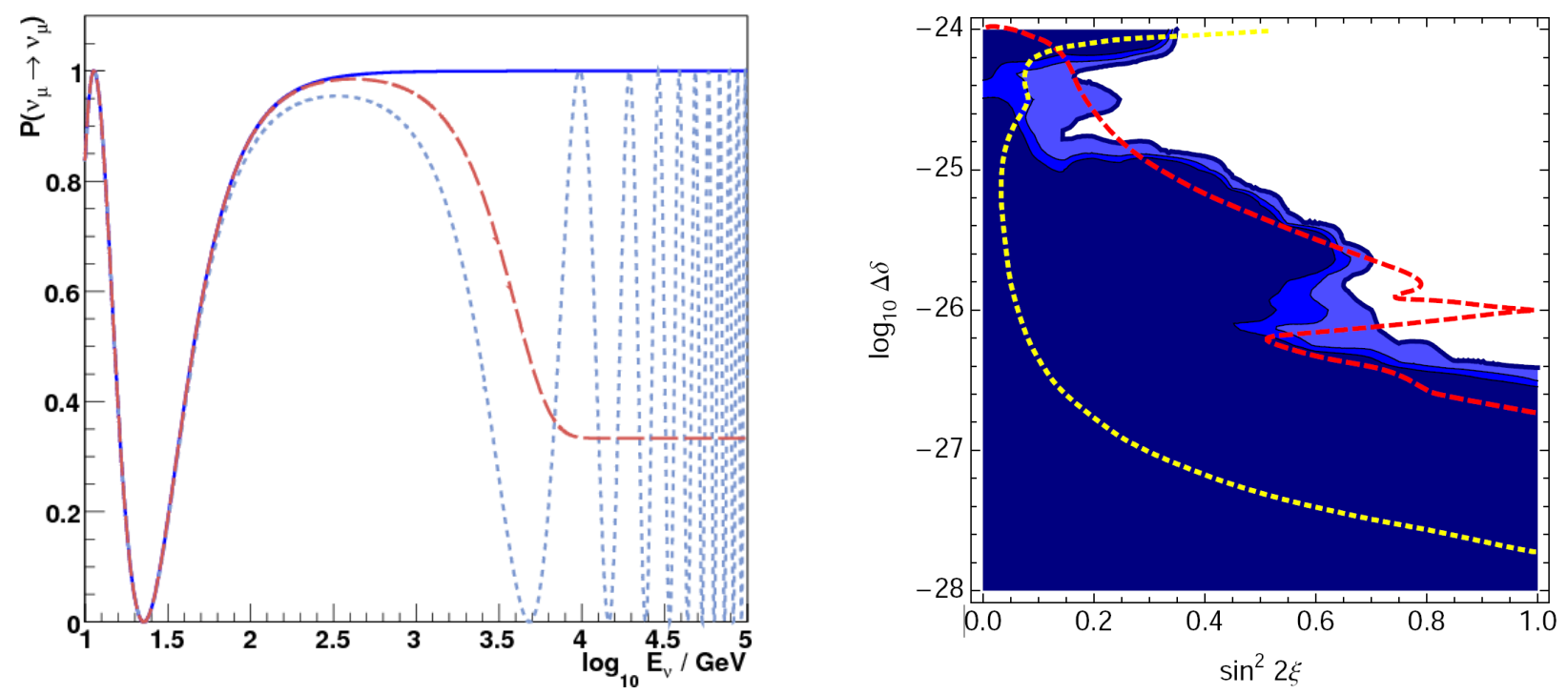

Figure 34. Left: $\nu_{\mu}$ survival probability as a function of energy for a baseline of the Earth diameter and for conventional oscillations (solid line), VLI (dotted line) with $n=1, \Delta \delta=10^{-26}$ and maximal mixing, and quantum decoherence (dashed line) with $n=2$ and $D_{i}^{*}=10^{-30} \mathrm{GeV}^{-1}$. See [98, for analysis methods taking into account the limited energy resolution. Right: allowed regions at $90 \%$, 95\% and 99\% confidence levels (from darkest to lightest) for VLI-induced oscillation effects $(n=1)$ from AMANDA data, together with the 90\%-contour from a combined Super-Kamiokande and K2K analysis (dashed line) and the projected IceCube 10-year 90\% sensitivity (dotted line). Figures are taken from [98].

Another exotic signature of VLI would be directional dependences of neutrino interactions or oscillations. Accelerator experiments like MINOS and K2K have searched for a sidereal modulation of their interactions rate (i.e. for a dependence on the orientation of their beam axes with respect to the Sun) and did not find any effect. A search for a sidereal effect of rates of atmospheric neutrinos in IceCube led to limits on the relevant parameters which improve MINOS/K2K limits by factors of 3-1000, due to the longer baseline and the higher energy (see [195] and references therein).

\subsection{Cosmic neutrinos}

High-energy cosmic neutrinos may either be identified as accumulation of events pointing to a particular celestial direction ("point sources") or as extended diffuse emission, ranging from a few degrees (as for nearby supernova remnants) to fully diffuse, expectedly isotropic neutrino flux; in both cases, the signal needs to be distinguishable from the intrinsic background of atmospheric neutrinos. 
Point-source searches use the directional and energy information to reduce this background. Cosmic neutrinos from a given source would cluster around the source direction, with a point spread function determined by the angle between muon and neutrino and by the detector angular resolution for muons, both depending on energy. A further handle in point source searches comes from the fact that generic extraterrestrial sources have a harder spectrum than atmospheric neutrinos. For variable sources the time distribution may be used as additional criterion, in particular if independent information is available (as e.g. gamma observations in the case of GRB signals).

Searches for diffuse fluxes can only use the measured energy as criterion for separating cosmic and atmospheric neutrinos. They thus critically depend on a detailed understanding of the detector response as a function of energy. Moreover, the high-energy tail of atmospheric neutrinos is dominated by prompt neutrinos, whose flux has larger uncertainties than that of conventional atmospheric neutrinos.

In the following, we will first summarise the results on diffuse fluxes obtained so far, then those for steady point sources, and finally methods and results for variable sources.

\subsubsection{Searches for diffuse cosmic neutrino fluxes}

A diffuse neutrino signal may reveal itself as an excess of

1. high energy upward moving muons from $\mathrm{TeV}-\mathrm{PeV}$ muon neutrinos;

2. high energy contained cascades;

3. extremely high energy events emerging from downward moving $\mathrm{PeV}-\mathrm{EeV}$ neutrinos of all flavours.

The first case corresponds to the standard signature of muon neutrinos interacting via charged current reactions. Due to neutrino oscillations, the expected ratio of neutrino flavours at Earth is $\nu_{e}: \nu_{\mu}: \nu_{\tau}=1: 1: 1$ (see Sect. 2.2.3). Since only charged current reactions of $\nu_{\mu}$ and, in about $17 \%$ of all cases, of $\nu_{\tau}$ produce high-energy final-state muons, whereas all other reaction channels appear as cascades, the relevance of detecting cascade events is obvious. The reduced directional accuracy (much worse for cascades than for muons) is not very important for diffuse fluxes.

For first-generation detectors, the ratio between the "fiducial" volume (within which cascades can be reliably identified) and the effective volume for muon detection is small. Therefore the cascade channel does not necessarily provide superior sensitivity for diffuse fluxes. This is particularly true for detectors in ice where light scattering makes the identification of cascades challenging. The situation is more favourable in water, where even events beyond the geometrical volume of the detector may be detected and reconstructed. Even without precise reconstruction of distant cascades, however, the non-observation of large signals from outside the geometrical volume can be used to derive upper limits on the diffuse flux. This approach has been pursued by the Baikal collaboration, who - in spite of the small volume of the NT200 detector - could compete with the much larger AMANDA detector over many years. 
For detectors on the cubic-kilometre scale, with a much smaller surface-to-volume ratio, the situation is more favourable for the cascade channel. The relevance of contained cascades for diffuse flux measurements is enhanced by the fact that their energy deposit in the detector volume - and thus their Cherenkov light output - correlates much more strongly with the neutrino energy than that of muons. Furthermore, the outer part of the instrumented volume can be used as a veto against events mimicking cascades.

No significant excesses over atmospheric neutrinos or other kinds of background has been observed so far, resulting in upper limits on the diffuse flux of extraterrestrial high energy neutrinos. Figure 35 summarises the limits obtained in the $\mathrm{TeV}-\mathrm{PeV}$ region. For each experiment and each method only the best limit is shown. Remarkably, from the first limit derived from the underground experiment Fréjus (1996) to the 2010 IceCube-40 limit, a factor of 500 improvement has been achieved. Several models as e.g. the blazar model of Stecker [57] shown in the figure can be excluded. A further factor of 10 improvement is expected over the next 2-3 years, using the full IceCube detector and combining muon and cascade information. The expected sensitivity is more than an order of magnitude below the Waxman-Bahcall bound, and prompt atmospheric neutrinos will be detectable for all but the lowest predictions [196]. Also, a test of the Waxman-Bahcall prediction for the diffuse flux of neutrinos from Gamma Ray Bursts [50] will be in reach. We note, however, that this model is much more easily tested with point source methods for transient, triggered sources (see below).

Neutrinos in the multi- $\mathrm{PeV}$ to $\mathrm{EeV}$ region must come from above or from close to the horizon to be detected since the Earth is not transparent for neutrinos of such energies (see Fig. 9 in Sect. 3.1). The IceCube collaboration has performed analyses which are tailored to these neutrinos of extreme energies and yield limits [193, 203] that meanwhile are equal or better than those obtained from radio or air shower detectors (see Sect. 7). Figure 36 summarises the differential limits in the $\mathrm{PeV}-\mathrm{EeV}$ range obtained by IceCube and by radio and air shower methods.

The figure demonstrates that IceCube can merely detect cosmogenic neutrinos from cosmic ray interactions with the cosmic microwave background radiation. Also Auger (with a multi-year exposure) and ANITA (with another flight) will have little chances to detect this "guaranteed source". Only dedicated detectors like e.g. next-generation radio or acoustic detectors or maybe a very large, sparsely instrumented configuration of KM3NeT will provide the required sensitivities. Arguably, other sources than cosmogenic neutrinos may populate the highest-energy region, making explorative studies in this energy range valuable. The alternative detection methods (radio, acoustic, air shower detection) are addressed in Sect. 7 .

\subsubsection{Searches for steady neutrino point sources}

In initial searches for point sources [209-211], the sky was subdivided into bins of a size which was optimised to the detector resolution and the expected signal energy spectrum (typically $E^{-2}$ ). In these "binned searches", the signal would appear as an excess over atmospheric neutrinos in a certain bin. In order not to lose sources through signal sharing between adjacent bins, the search had to be repeated with shifted bins, resulting in trial factors which effectively reduced the sensitivity. Present searches, in contrast, use likelihood 


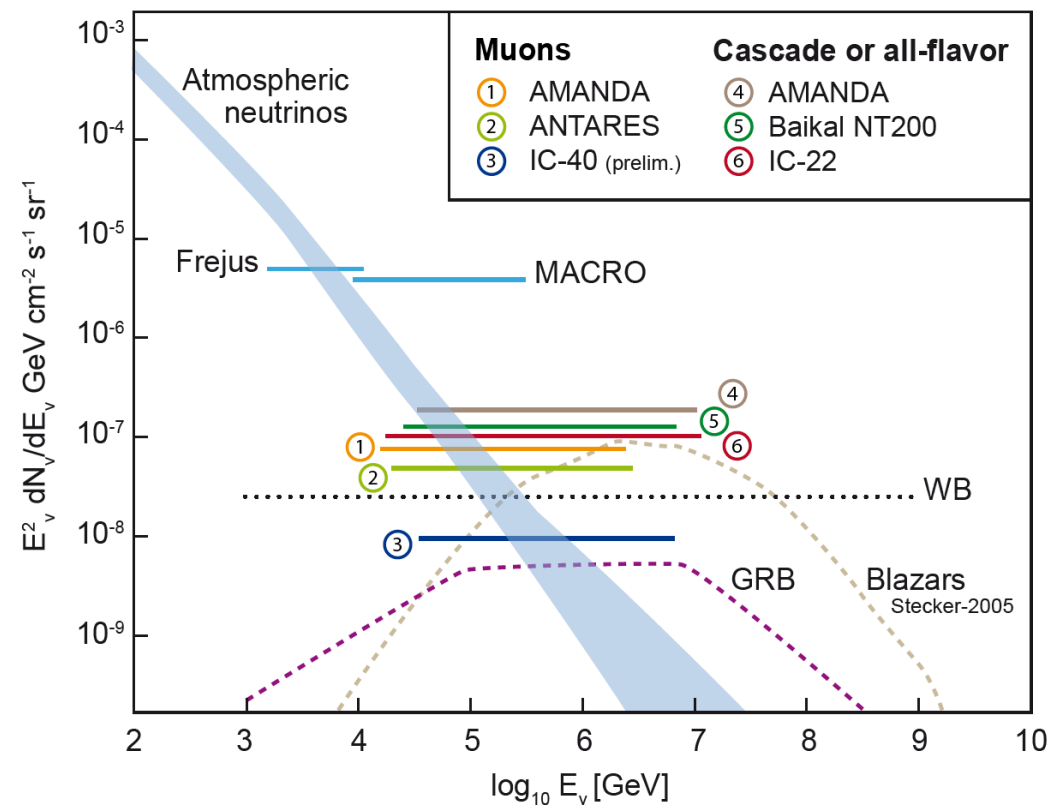

Figure 35. 90\% C.L. integral upper limits on the diffuse flux of extraterrestrial neutrinos. The horizontal lines extend over the energy range which would cover $90 \%$ of the detected events from an $E^{-2}$ source ( $5 \%$ would be below and $5 \%$ above the range). All model predictions have been normalised to one flavour, i.e. all of the all-flavour limits have been divided by 3 . The coloured band indicates the measured flux of atmospheric neutrinos (see also Fig. 33), the broadening at higher energies reflects the uncertainties for prompt neutrinos. The limits on muon neutrinos are from 807 days AMANDA [197, 334 days ANTARES [198], and 375 days IceCube-40 (preliminary). Cascade/all flavour limits are from 807 days AMANDA [199], 1038 days Baikal-NT200 [140, 200], and 257 days IceCube-22 [201]. The Fréjus and MACRO limits have been published in [11] and [202], respectively. Also indicated is the Waxman-Bahcall (WB) bound [60], see Sect. 2.1.4.

functions which account for the smearing of the signal with a given point spread function. The probability of an event originating from a given source is calculated from a 2-dimensional probability density (typically a Gaussian), with the width defined by the uncertainty of the event direction. This "unbinned method" turned out to be up to $40 \%$ more sensitive than the binned [212]. Using an energy estimator to distinguish hard extraterrestrial from soft atmospheric neutrino spectra further enhances the sensitivity.

The upper limit on a $E^{-2}$ neutrino flux from any point source in the Northern sky measured with AMANDA over seven years is $E^{2} \phi<5.2 \times 10^{-8} \mathrm{GeV} \mathrm{cm}^{-2} \mathrm{~s}^{-1} \mathrm{sr}^{-1}$ (averaged over the Northern hemisphere, using a binned method) [211]. Based on 813 days of data taking, ANTARES has recently released a sky map and preliminary values [213] for the flux limits of a set of preselected potential point sources (see Fig. 37), which improve the Southern sky limits from 14 years of Super-Kamiokande data.

The real big leap, however, is being made with IceCube. First point source results were published for the 22-string configuration operated in 2007 [215]. In [216] this search 


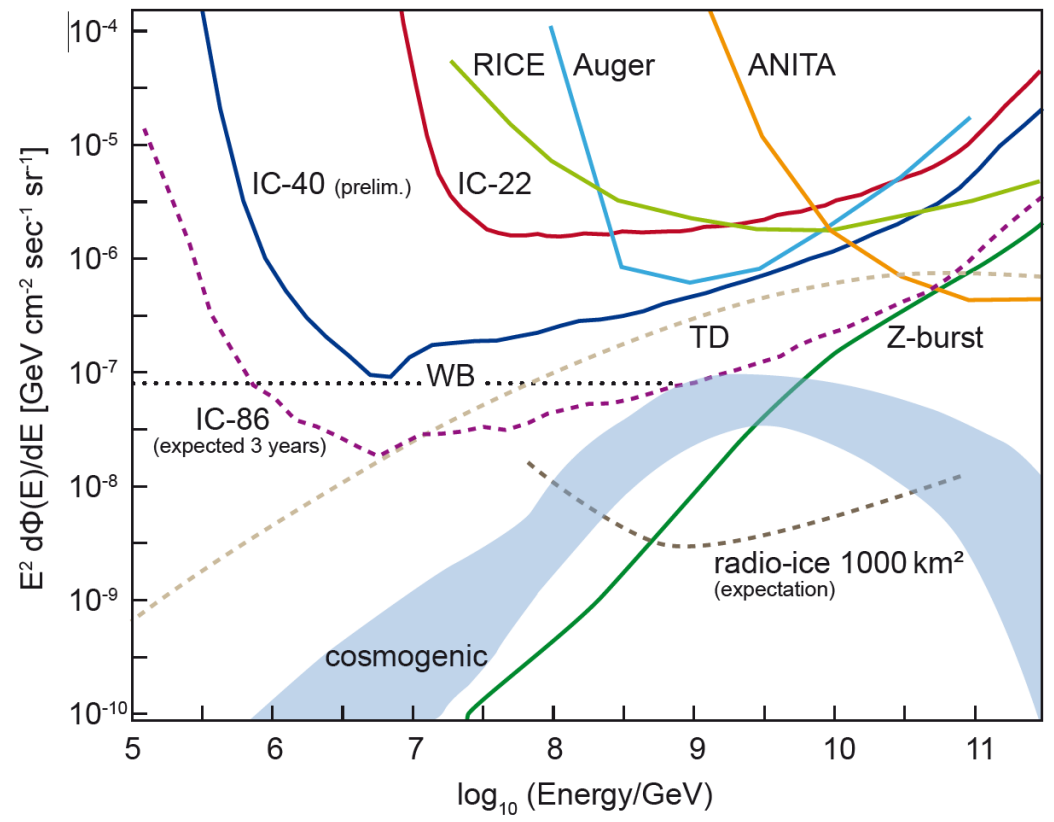

Figure 36. All-flavour 90\% C.L. differential upper limits on the flux of extraterrestrial neutrinos in the $\mathrm{PeV}-\mathrm{EeV}$ region. Limits are from the under-ice radio array RICE [204], the air shower detector Auger [205], the radio balloon experiment ANITA [206], IceCube-22 [193], and IceCube-40 (preliminary) 203. Also given are the expectations for 3 years of operation of the full IceCube detector and for a $1000 \mathrm{~km}^{2}$ shallow radio detector at the South Pole. The coloured band corresponds to different predictions for cosmogenic neutrinos from GZK processes. References for the GZK scenarios, the Z-burst prediction and the SUSY top-down scenario (TD) can be found in [203]. For recent predictions we refer to [64, 207, 208].

was extended up to $45^{\circ}$ above the horizon. The background from down-going atmospheric muons was suppressed by hard cuts selecting only few thousand of the billions of background events, including most of the $\mathrm{PeV}-\mathrm{EeV}$ neutrinos. In that way, a reasonable sensitivity to the high-energy tail of hard neutrino spectra (e.g. $E^{-2}$ ) was achieved. Naturally, the IceCube sensitivity to a corresponding source in the Southern hemisphere is worse than for Northern sources since the analysis relies exclusively on the tiny high-energy tail of the neutrino flux. Note, however, that IceCube would be almost blind to Southern sources with an energy cut-off in the PeV range or below (some remaining sensitivity is expected from DeepCore, which, using the rest of IceCube as a veto layer, could identify neutrinos which interact within the DeepCore volume with $4 \pi$ acceptance). For unbroken $E^{-2}$ spectra, a cubickilometre detector at the South Pole can compete with a Northern first-generation detector like ANTARES up to a declination of $45^{\circ}$. This, on the other hand, means that there is a broad declination region where the combination of IceCube and ANTARES data will give a better sensitivity than IceCube or ANTARES alone. Such combined analyses are presently underway.

Figure 38 shows the full-sky map derived from IceCube-40 data 214 taken between April 

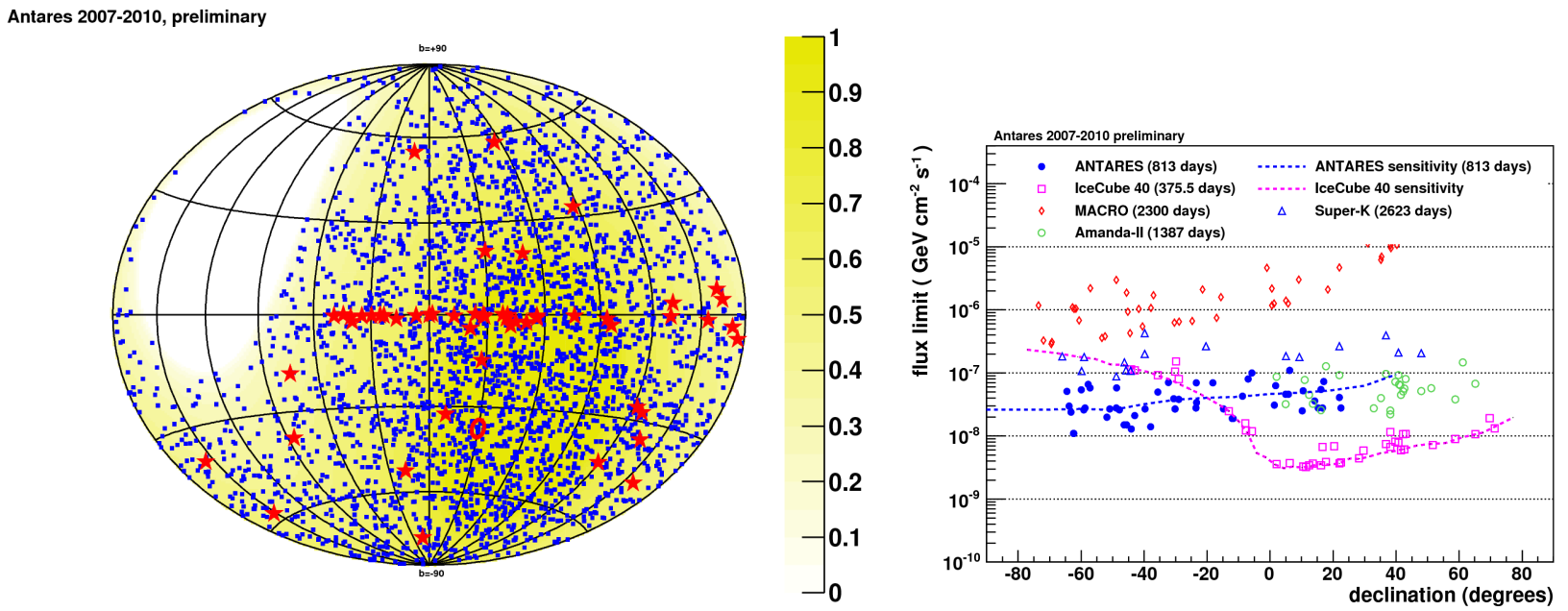

Figure 37. Left: Equatorial skymap of neutrino-induced muon events from 813 days of ANTARES data from 2007-2010 (preliminary). The background colour scale indicates the sky visibility in percent of the time. The most significant accumulation of events, marked with a red circle, is compatible with the background expectation. Right: Flux limits for the 51 candidate sources marked in the skymap, compared to the expected average ANTARES sensitivity (blue dotted line) and to corresponding results from MACRO [209], Super-Kamiokande [210, AMANDA [211] and IceCube [214].

2008 and May 2009. The data sample contains 14121 events from the Northern sky, mostly muons from atmospheric neutrinos, and 22779 events from the Southern sky, mostly high energy atmospheric muons. With this sample, five searches were performed: (i) a scan of the entire sky for point sources; (ii) an analysis for 39 predefined potential sources (reducing the huge trial factor inherent to a full-sky search); (iii) the remaining three analyses stacked source candidates of different astrophysical nature and then searched for an excess. These classes were (iii-a) 16 sources of TeV gamma rays; (iii-b) 127 starburst galaxies and (iii-c) 5 nearby galaxy clusters. The predefined sources included, among others, the Crab Nebula, the Geminga pulsar, the star cluster Cygnus OB2 and the active galaxies Markarian 421, Markarian 501, 3C273 and M87. The results of the predefined point-source search (ii) are shown in Fig. 37 (right). All search results are consistent with the null hypothesis; the "hottest spot" from the all-sky scan is found at a right ascension of RA $=113.75^{\circ}$ and a declination of $\mathrm{DEC}=15.15^{\circ}$ and has a chance value of $18 \%$. Figure 39 compiles the limits from previous experiments, from the different IceCube stages and the expected sensitivity of one year of KM3NeT data. We note that the combined data of IceCube-40 and IceCube-59 surpass the mark of $1 \mathrm{~km}^{3} \times 1$ year and thus exceed 1 year worth of data from the full IceCube detector. Very soon, a factor of 1000 improvement of the sensitivity to point sources will have been reached when compared to the very first AMANDA point source paper from 2000 [217.

Where do we stand with respect to predictions for known sources? Figure 40 gives a "taste" of the answer. It shows the differential fluxes for three theoretical models and confronts them with the $90 \%$ C.L. upper limit and the $5 \sigma$ discovery potential from IceCube- 

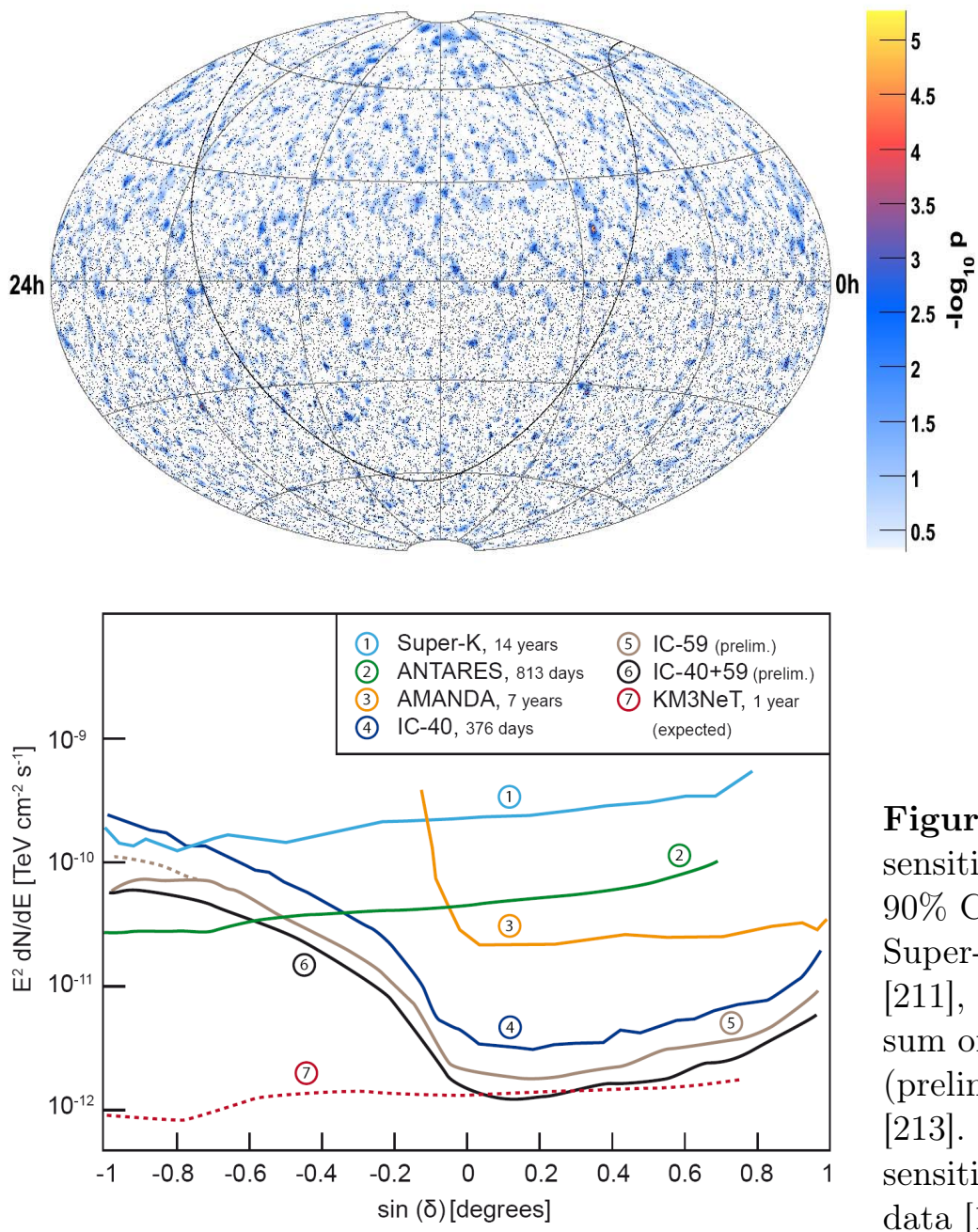

Figure 38. Equatorial skymap of pre-trial significances of the all-sky point source search with IceCube-40 [214]. Each dot represents one neutrino event, the colour scale indicates the significance of event accumulations. The Galactic plane is shown as black curve.

Figure 39. Point source neutrino flux sensitivities (median expected limits at 90\% C.L.) from various experiments: Super-Kamiokande [210], AMANDA [211], IceCube-40 [214], IceCube-59, the sum of IceCube-40 and IceCube-59 (preliminary results) and ANTARES [213. Also shown is the expected sensitivity from one year of KM3NeT data 103 .

40. Its shows the $\nu_{\mu}+\bar{\nu}_{\mu}$ predictions for the supernova remnant RX-J1713.7-3946 [218] which was moved from its real position at the Southern sky the to the location of the Crab Nebula at the Northern sky, for MGRO J182+01 [39], a gamma-ray source observed by the Milagro experiment, and for the active galaxy Centaurus A [219]. Note that recent measurements from the Fermi satellite [31] seem to disfavour a dominant hadronic origin of the gamma rays observed from RX-J1713.7-3946 - the underlying assumption for the shown prediction (see also Sect. 2.1.3). The conclusion is that optimistic model predictions are about one order of magnitude below present IceCube-40 limits. This does not rule out a discovery with a few years of full IceCube data. On the other hand, it seems that with IceCube after having made the gigantic leap of a factor 1000 in sensitivity improvement - we are just scraping the discovery region. The main discovery potential therefore may remain for source phenomena which are not covered by the models addressed in Fig. 40, e.g. "dark" sources without significant high-energy gamma emission, or variable sources discussed in Sect. 6.2.3. The implications for a Northern-hemisphere detector, however, are obvious: Its sensitivity must substantially exceed that of IceCube! 


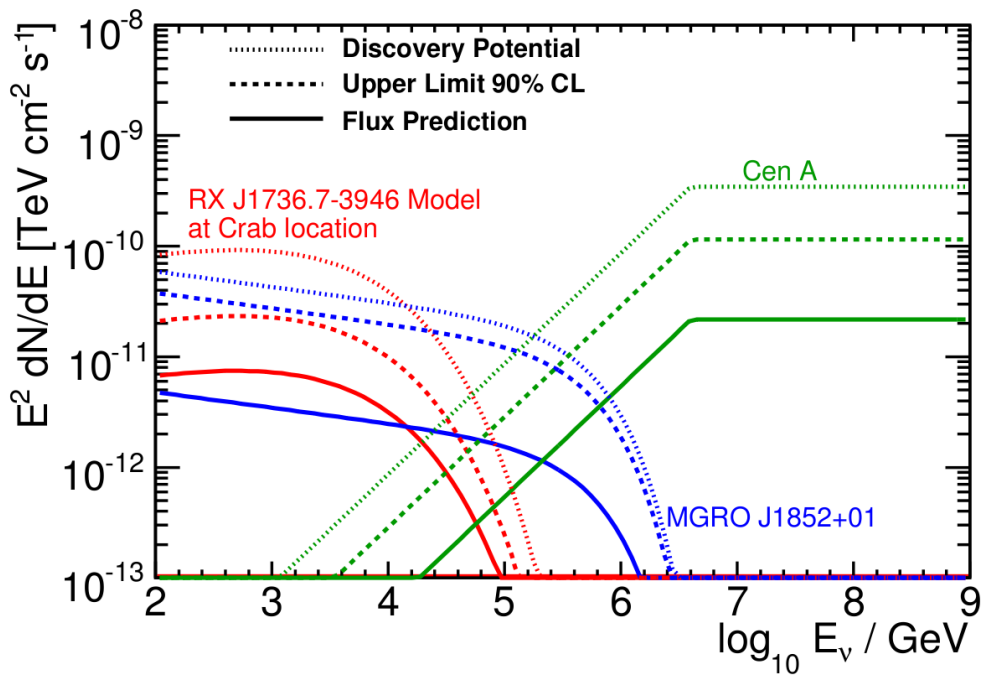

Figure 40. Differential flux for three theoretical models of neutrino point sources, confronted with the $90 \%$ C.L. upper limit and the $5 \sigma$ discovery potential from IceCube-40 (see text for explanations). Figure taken from [214.

\subsubsection{Searches for variable neutrino sources}

Many astrophysical sources are known to have a variable flux at different wavelengths. Examples for such flaring sources are Active Galactic Nuclei, Soft Gamma Ray Repeaters, and Gamma Ray Bursts. Binary systems often show a periodic behaviour, as pulsars do. Neutrino searches for steady point sources integrate over time and continuously accumulate atmospheric neutrinos. That makes them less sensitive to the detection of flares when compared to searches over smaller time windows. Actually, compared to searches integrating over a full year, time-dependent searches can achieve $5 \sigma$ discoveries with two (five) times less signal events for flares of 1 day ( 1 second) duration [220 222] - even if the there is no information on the flaring state from electromagnetic observations (so-called "untriggered" searches). In case this information is available and used ("triggered searches"), the gain can even be larger, in particular for Gamma Ray Bursts signalled by satellite detectors.

IceCube has performed an untriggered search for flares from selected sources using IceCube-40 data (April 2008 to May 2009), and a triggered search for sources monitored by Fermi-LAT, SWIFT and Imaging Cherenkov Telescopes using IceCube-22 and IceCube-40 data (May 2007 to May 2009) [223]. These analyses cover time intervals from $40 \mu \mathrm{s}$ to a year. No significant signal evidence was found.

In this context it may be worth mentioning that of seven flares which were selected for the triggered search with IceCube-22, five did not show a related neutrino signal in the selected time window, while one event was observed for each of the other two sources. One of these sources is the Active Galaxy 1ES1959+650, which has a "neutrino history": In an analysis of AMANDA data taken from 2000-2003, five events where recorded from the direction of 1ES1959+650. Interestingly, three of these came within 66 days in 2002. Two of the three neutrinos were coinciding within about a day with gamma-ray flares observed by the gamma-ray telescopes HEGRA and Whipple [224, 225]. Excitingly, one of these 2 flares was not accompanied by an X-ray flare; such "orphan flares" would be expected for hadronic outbursts where the X-ray flux from synchrotron radiation of the electron plasma 
is absent. This result was quickly followed by two theoretical papers, one claiming that the corresponding neutrino flux would not fit any reasonable assumption on the energetics of the source [226], the other claiming that scenarios yielding such fluxes were conceivable [227]. Since the analysis was not a fully blind analysis, it turned out to be impossible to determine chance probabilities for this event, and actually the result was never published in a journal. However, it initiated consideration to send alerts to gamma-ray telescopes in case time-clustered events from a certain direction would appear. These "Target-of-Opportunity" alert programs are described below.

A special analysis [228] was performed for SN 2008D, a core-collapse supernova at a distance of $27 \mathrm{Mpc}$ discovered by the SWIFT satellite on Jan. 9, 2008. Core-collapse supernovae might emit mildly relativistic jets (Lorentz factor $\Gamma=3-10$ ) in which neutrinos could be produced by proton-proton collisions. Assuming that the jet pointed to the Earth, the non-observation of coinciding neutrinos with IceCube was used to constrain the total energy of the released energy and $\Gamma$. Actually, according to current models, the full IceCube detector could detect up to 100 events for a core-collapse supernova at $10 \mathrm{Mpc}$ distance.

One of the most promising sources of high energy neutrinos are Gamma Ray Bursts (GRBs). As mentioned in Sect. 2.1.4. neutrino emission can be modeled for three GRB phases: the precursor phase when the jet is still forming and no electromagnetic radiation is escaping [51]; the prompt phase coinciding with the burst in gamma rays (see e.g. [50, 229]); and the afterglow phase [52. Direction, time, duration and gamma spectrum of GRBs are provided by satellite observations. Early searches for neutrino events coinciding with GRBs have been performed with Super-Kamiokande, Baikal and AMANDA. The AMANDA analysis [230] looked for neutrinos coinciding with any of 408 well-located GRBs recorded in the Northern hemisphere between 1997 and 2003. No neutrinos were found during or immediately prior to the GRBs. An upper limit on the diffuse flux from all GRBs was derived, which was still a factor of 1.5 above the flux predicted in [50] for the prompt phase, but already two times below the precursor model published in [51. In a recent analysis, neutrinos recorded with IceCube-40 have been analysed with respect to coincidences with any of 117 Northern-hemisphere GRBs recorded in the time interval April 2008 to May 2009 [231]; preliminary results for IceCube-59 are also already available [232]. Differently to the AMANDA analysis, the expected neutrino spectra have been calculated according the the observed gamma spectra from each individual GRB and following the prompt phase model of [229]. Again no excess of neutrinos close in time and direction is observed, resulting in a limit below the predictions of [50, 229], see Fig. 41. Even though this does not rule out the general picture of the GRB fireball model of [50] which assumes that cosmic rays of highest energy essentially all emerge from GRB, the amount of energy of the accelerated protons transfered to pions is obviously smaller than assumed in these models. The transfer parameter is uncertain by a factor of 3 or more; this and other uncertainties in the model parameters leave room for a possible confirmation of these models with future IceCube data.

\subsubsection{Alert programs}

Neutrino telescopes monitor essentially a full hemisphere. In contrast, most gamma-ray, X-ray and optical observatories can observe only a small fraction of the sky at any given 


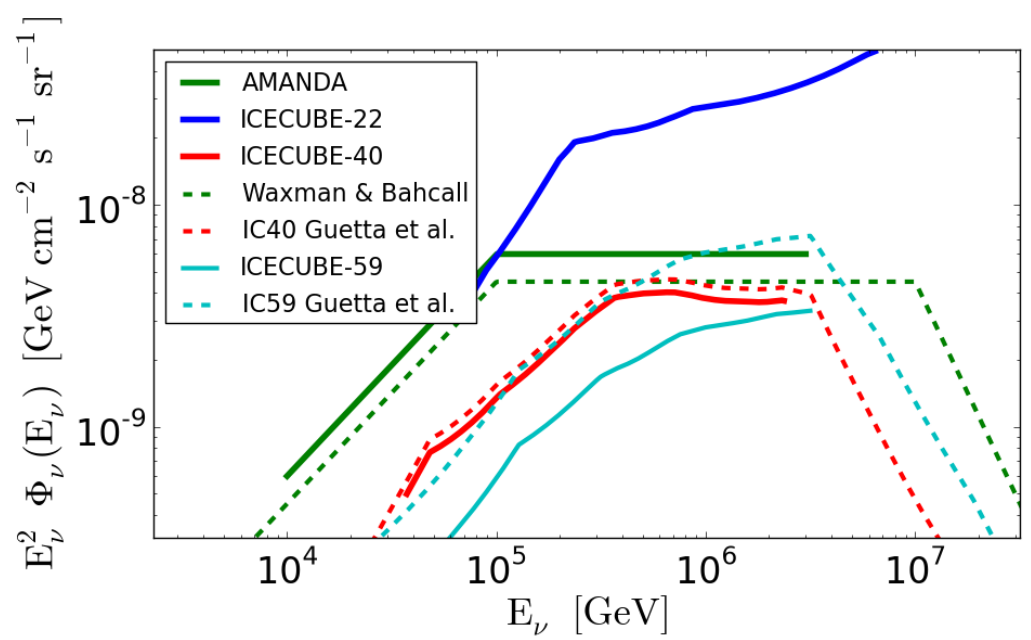

Figure 41. 90\%C.L. upper limits on the neutrino fluxes from GRBs set by AMANDA, IceCube22 , IceCube-40 and IceCube-59 (preliminary). The IceCube limits are calculated with respect to the flux expected from the model of Guetta et al. 2229], the AMANDA limit with respect to the Waxman-Bahcall flux [50] which assumes an average shape of the GRB spectra. The fluence limits for single GRBs have been translated to a limit on the diffuse neutrino flux from GRBs, multiplied with $E^{2}$. The corresponding model predictions are indicated as dashed lines. The strikingly worse limit of IceCube-22 as compared to AMANDA and later IceCube configurations is due to small statistics and one observed conicidence. Figure adapted from [232].

moment. Therefore only a small subset of neutrino data can be used for correlation studies with these data. The ability to identify such correlations can be improved by optical followup (OFU) programs [233 235]. In these programs, the observation of doublets or triplets of neutrino events from the same direction (or one exceptionally energetic single neutrino event) triggers a small network of automated 1-2 metre telescopes to point in that direction. If the neutrinos are emitted from jets in core-collapse supernovae in other galaxies or from GRBs, the optical telescopes could identify the rising light emission from the supernova or the GRB afterglow. As was shown in [233], OFUs can improve the sensitivity to neutrinos from supernovae and GRBs by a factor of 2-3. Both IceCube and Antares are running OFU programs, triggering the optical telescopes TAROT and ROTSE (for details see [235]. No coincidences have been reported so far, although the IceCube analysis provided some interesting (but not significant) doublets. Presently, these programs are extended to include the X-ray satellite SWIFT.

Another follow-up program in IceCube is known as Neutrino Target of Opportunity (NToO) [236]. It was motivated by the coincidence in 2002 between two neutrinos from the direction of the AGN 1ES1959+650 and gamma ray flares from the same source (see Sect. 6.2.3). In this case, the problem is complicated by the comparatively long duration of AGN flares (hours to weeks instead of seconds to minutes for supernovae or GRBs). Doublets within such long time intervals appear too often from any direction of the sky to be useful for 
such a program. Therefore only selected sources, which are known to show flaring behaviour, are used for the NToO. In case a "cluster in time" of events from the direction of one of these sources has accumulated, a trigger is sent to the MAGIC gamma-ray telescope in La Palma. As soon as possible MAGIC is then pointed to that direction.

A further spectacular alert program is the "standard" IceCube supernova alert. As mentioned in 5.1.3, the low dark noise rate of the photomultipliers allows for a mode of operation which is best suited for ice: the detection of burst neutrinos via the feeble increase of the summed count rates of all photomultipliers. This increase would be produced by millions of neutrino interactions at energies up to some tens of $\mathrm{MeV}$ within several seconds [179, 237]. The tiny amount of light from one of these interactions would usually fire only the closest photomultiplier, so that no event-by-event reconstruction is possible. With this method, a supernova in the centre of the Galaxy would be detected with extremely high confidence, and the onset of the pulse could be measured in unprecedented detail since IceCube records the counting rate in millisecond steps. Even a SN 1987A-type supernova in the Large Magellanic Cloud would be identifiable.

Since neutrinos leave a supernova a few hours before light is emitted, neutrino signals can be used to issue an early alert to optical astronomers. Actually, IceCube alerts are fed into the SuperNova Early Warning System, SNEWS [181]. Currently, the detectors SuperKamiokande (Japan), LVD and Borexino (Italy) as well as IceCube contribute to SNEWS, with a number of other neutrino detectors and gravitational wave detectors planning to join in the near future.

The average supernova rate for a galaxy like ours is estimated to be $2-5$ per century (see [238] for an overview). Since 1987 no supernovae have been observed in our Galaxy or its neighbourhood. There might have been, however, Galactic supernovae obscured by matter between them and us which consequently could have been visible only in neutrinos. The non-observation of low-energy neutrino signals by underground neutrino detectors like Super-Kamiokande and by AMANDA/IceCube sets a 90\% C.L. upper limit of about eight per century on the average rate of such bursts (we have rescaled the 2007 limit obtained in [238] for 25 years of non-observation to meanwhile 29 years).

\subsection{Dark matter and other exotic particles}

In addition to neutrinos from astrophysical objects, as discussed above, neutrino telescopes are also sensitive to potential neutrino fluxes from dark matter annihilations (indirect dark matter searches) and to hypothesised exotic particles (see Sect. 2.2). The status of the corresponding experimental search results and the future expectations are discussed in this section.

\subsubsection{Dark matter}

As explained in Sect. 2.2, dark matter could accumulate in the Galactic centre, in the Sun or in the centre of the Earth and eventually release neutrinos via self-annihilation processes. These neutrinos would be detectable as an excess over the irreducible background of atmospheric neutrinos. Several underground and underwater/ice experiments (Baksan, MACRO, Super-Kamiokande, Baikal, AMANDA, IceCube (see [239] and references therein) 
as well as ANTARES [240-242]) have searched for an excess of neutrinos from the centre of the Earth or the Sun, IceCube also for signals from the Galactic halo and the Galactic centre [243, 244] - all without having identified any significant signal. Alternative indirect searches using gamma rays [245] or other particle messengers [246] have not found clear dark matter signals either.
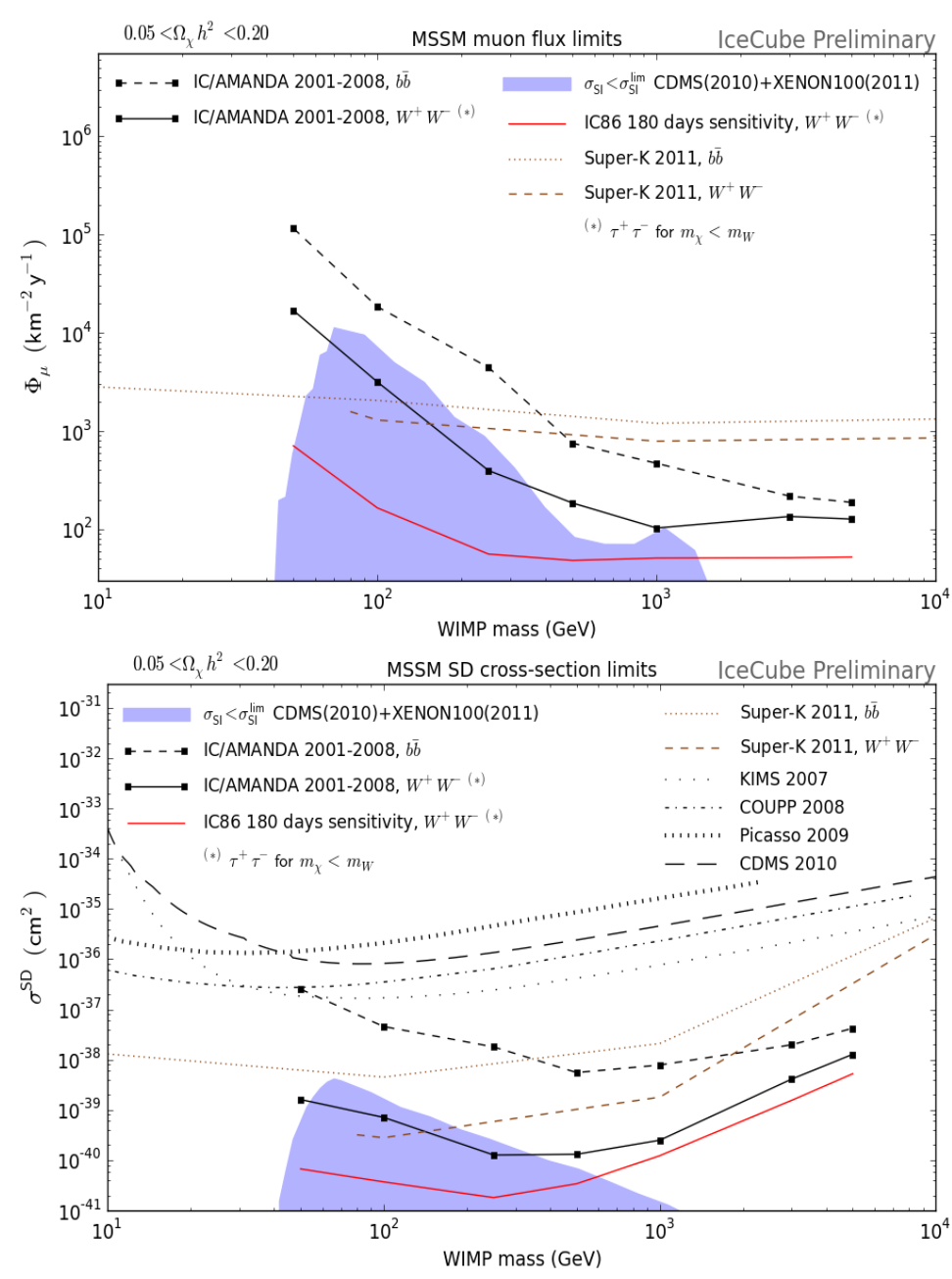

Figure 42. 90\% C.L. exclusion limits on the muon flux induced by neutrinos from neutralino annihilations in the Sun (top) and on the spin-dependent neutralino-proton cross section (bottom) from indirect dark matter searches in the neutrino channel, as functions of the WIMP mass. The shaded areas indicate the MSSM parameter regions not excluded by direct searches (see text). Indicated are the limits obtained with AMANDA, IceCube-22 and IceCube-40 (squares) assuming soft $(b \bar{b})$ and hard $\left(W^{+} W^{-}\right)$neutrino spectra from WIMP annihilations; the expected sensitivities of IceCube with DeepCore (red lines); and exclusion limits obtained by other experiments. Limits have been rescaled to a common muon threshold of $1 \mathrm{GeV}$. See 244] for references to the various experimental data; the Super-Kamiokande results are taken from [247].

The most sensitive search for neutrino-induced muons from the direction of the Sun has been performed with IceCube-22, using data taken between March and September 2007 [244]. In these months, the Sun is below the horizon at the South Pole. Simulations have been run for several neutralino masses each with two annihilation channels, a hard channel (annihilation into $W^{+} W^{-}$), and a soft channel (annihilation into $b \bar{b}$ ). Figure 42 (left) shows the limits on the flux of muons induced by neutrinos produced in MSSM neutralino annihilations in the centre of the Sun as a function of the neutralino mass $m_{\chi}$. Since the average energy of the muons would increase with neutralino mass and hardness of the decay channel, and since the muon detection probability increases with energy, the limits are tighter for harder decay spectra and for larger $m_{\chi}$. The green shaded area represents flux 
predictions from presently allowed combinations of the MSSM parameters based on direct search experiments. The grey shaded area represents the parameter space that would still be allowed if direct searches would improve their sensitivity by a factor of 1000 .

Since the muon flux is proportional to the capture rate of neutralinos in the Sun, the muon flux limit can be converted into a limit on the neutralino-proton scattering cross section, in particular its spin-dependent part, $\sigma_{\chi+p}^{S D}$ (the Sun is mainly a proton system). In [244, this conversion has been performed by assuming equilibrium between capture and annihilation rates. A conservative result is obtained by further assuming that the spinindependent cross section vanishes. The result of this analysis is given in Fig. 42 (right) and compared to other limits from indirect searches by Super-Kamiokande and IceCube-22 as well as the best current limits from direct searches. The figure illustrates the potential of indirect searches to explore the parameter space of spin-dependent neutralino interactions with a sensitivity exceeding by far that of direct searches.

Using the same method, data from AMANDA and IceCube have been used to set limits on other dark matter candidates, like the lightest Kaluza-Klein particle (hypothesised in theories with extra dimensions, with an expected mass range similar to that for neutralinos) and Simpzillas (super-heavy relic particles with masses in the range of $10^{4}-10^{18} \mathrm{GeV}$ ). We refer to [244, 248] for details.

An alternative approach was pursued to estimate the KM3NeT sensitivity to neutrinos from dark matter annihilations. The mSUGRA parameter space was scanned; for each parameter set the resulting neutrino spectrum was calculated and the resulting event rate in KM3NeT determined, taking properly into account neutrino propagation through the Sun. The resulting limits for the spin-dependent cross section, equivalent to Fig. 42 (right), are shown in Fig. 43 (see [103] for further explanations and references to the experimental data). KM3NeT in the currently planned configuration (see Sect. 5.2), with its comparably high detection threshold, is less sensitive to indirect dark matter signals than IceCube with DeepCore.

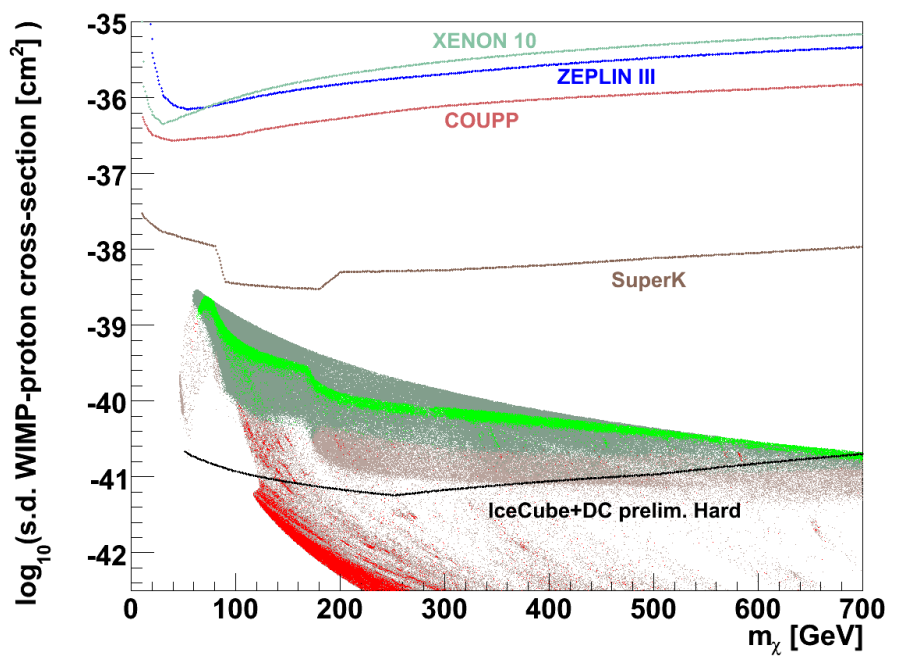

Figure 43. Spin-dependent WIMP-proton cross section as a function of the neutralino mass. The coloured points correspond to mSUGRA parameters consistent with constraints from cosmic microwave measurements. The models within (beyond) the discovery reach of KM3NeT are coloured green (red). Limits of selected other experiments are also indicated. The KM3NeT and IceCube limits are for 10 years of data taking. 


\subsubsection{Magnetic monopoles, Q-balls and nuclearites}

In water and ice detectors, relativistic magnetic monopoles can be identified via their strong light emission, 8300 times more intense than that of a minimally ionising muon. Even below the Cherenkov threshold (velocity $v_{c} \approx 0.75 c$ ), down to $v \approx 0.5 c$, the light emission is large due to accompanying delta electrons. Figure 44 shows the monopole flux limits obtained by Baikal, AMANDA and ANTARES, where only in the ANTARES analysis light emission below $v_{\text {mon }}=0.75 c$ was addressed. The Baikal flux limit of $4.7 \times$ $10^{-17} \mathrm{~cm}^{-2} \mathrm{~s}^{-1} \mathrm{sr}^{-1}$ for upward-moving monopoles with $\beta \approx 1$ is based on 1040 live days of NT200 (1998-2002) [249]; that of AMANDA of $2.8 \times 10^{-17} \mathrm{~cm}^{-2} \mathrm{~s}^{-1} \mathrm{sr}^{-1}$ for upward-going and $3 \times 10^{-16} \mathrm{~cm}^{-2} \mathrm{~s}^{-1} \mathrm{sr}^{-1}$ for downward-going monopoles on the data taken with AMANDA in a single year (2000) [250]; the preliminary ANTARES limit for upward-moving monopoles with $\beta_{\text {mon }} \approx 1$ is at $1 \times 10^{-17} \mathrm{~cm}^{-2} \mathrm{~s}^{-1} \mathrm{sr}^{-1}$ [251]; the preliminary IceCube limit derived from one year of data taking with the 22-string configuration is about $3 \times 10^{-18} \mathrm{~cm}^{-2} \mathrm{~s}^{-1} \mathrm{sr}^{-1}$ [252. IceCube in its full configuration will be yet another factor 3-4 more sensitive and thus advance to more than three orders of magnitude below the Parker bound [253], which is set by the condition that magnetic monopoles must not destroy the Galactic magnetic fields. Note that due to the absence of predictions for magnetic monopole fluxes a non-observation cannot exclude certain theoretical models (as it is the case for dark matter searches).

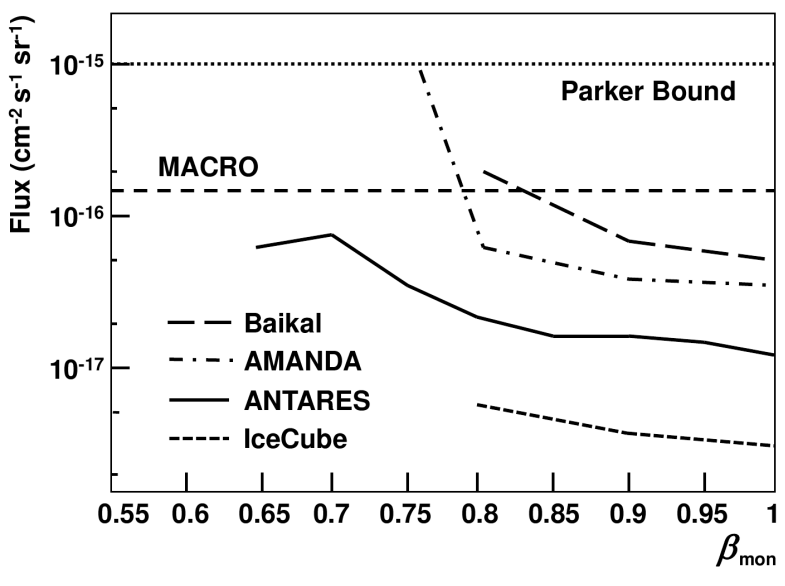

Figure 44. 90\% C.L. upper limits on the flux of relativistic magnetic monopoles as a function of their velocity, $\beta_{\text {mon }}=v_{\text {mon }} / c$. The ANTARES and IceCube results are preliminary.

Slow GUT monopoles, Q-balls and nuclearites (see Sect. 2.2) would heavily ionise the matter they traverse, or even catalyse proton decays along their path. Such particles with $\beta$ in the range $10^{-5}-10^{-2}$ have been searched for with underground and underwater/ice detectors; in absence of any signals, upper limits on their fluxes of the order of $10^{-16} \mathrm{~cm}^{-2} \mathrm{~s}^{-1} \mathrm{sr}^{-1}$ are derived [254 257]. Note that limits based on the catalysis of proton decay are conditional since they depend on an assumed catalysis cross section. For instance, the limit of the Baikal experiment on GUT monopoles is $2[50] \times 10^{-16} \mathrm{~cm}^{-2} \mathrm{~s}^{-1} \mathrm{sr}^{-1}$ for catalysis cross sections of $10^{-28}\left[10^{-30}\right] \mathrm{cm}^{2}$ and $\beta=10^{-4}$. Again, IceCube data will improve this sensitivity by about two orders of magnitude. Needless to mention that intense light emission of a slow object would be a spectacular signature which would make the first clear observation of such an event a real sensation - regardless of its concrete interpretation as GUT monopole, Q-ball or nuclearite. 


\subsection{Cosmic ray physics}

In detectors underwater/ice and underground, cosmic rays are recorded via the punchthrough muons generated in air showers above the detector. Ideally, the deep detector is complemented by a surface air shower array for such measurements. In this case, the air shower array essentially responds to the total energy contained in the electromagnetic shower component, whereas the deep detector responds to the hadronic shower component from which the muons originate. Combination of both pieces of information allows for disentangling energy spectra and mass composition of the primary particles on a statistical basis. This approach has been pioneered with underground/air shower hybrid detectors like Baksan/Carpet (Russia) or MACRO/EAS-Top (Italy), followed by AMANDA/SPASE (South Pole Air Shower Experiment) and recently IceCube/IceTop (both at South Pole). The experimental results indicate that the average mass of the primary particles increases with energy in the knee region around $10^{15} \mathrm{eV}$, see [258] and references therein. The combination IceCube/IceTop covers an enormously enlarged solid angle and an area of a full square kilometre, and yields data of unprecedented quality. With its huge statistics it will extend the energy range up to $10^{18} \mathrm{eV}$, where the transition from Galactic to extragalactic cosmic rays is expected.

Surface detectors can also be used to estimate the angular resolution of the deep detector, by comparing the reconstructed directions of air showers and the corresponding muon. Comparing data from SPASE and AMANDA, the muon angular resolution of AMANDA was measured to be $2^{\circ}-2.5^{\circ}$ [259]. The angular resolution of IceCube was determined via the shadow of the moon with respect to cosmic rays. This shadow can be measured via down going muons with a high significance within a few weeks, and confirms that angular resolution and absolute pointing of IceCube are about $1^{\circ}$ or better [260].

One of the initial motivations for deep-water detectors was to clarify puzzles in the depthintensity relations that had emerged from underground muon measurements (see Sect. 4.1. Due to the energy dependence of the muon range in matter, these measurements are sensitive to the energy spectrum of muons from cosmic-ray-induced air showers. Figure 45 261 shows a compilation of recent data, dominated by muons with energies below $100 \mathrm{TeV}$; the measured muon flux excellently follows a model prediction [262], thus demonstrating that this aspect of cosmic ray physics is well under control in the energy range covered.

Over the last years, however, a new cosmic ray puzzle has emerged: Several detectors located at the Northern hemisphere have found tiny anisotropies in the arrival direction of cosmic rays in the range of several $\mathrm{TeV}$ to several hundreds of $\mathrm{TeV}$ (Milagro [263], Tibet air shower array [264, ARGO/YBJ [265]) and of down-going atmospheric muons (SuperKamiokande [266]). Using recorded data of several $10^{10}$ down-going muons with energies above a few $\mathrm{TeV}$, IceCube has detected per-mille anisotropies on all angular scales down to about $15^{\circ}$. A multipole analysis yields the strongest power for dipole $(l=1)$ and quadrupole $(l=2)$ contributions. The dipole does not point to the direction suggested by the ComptonGetting effect (anisotropy induced by the movement of the Earth [267]) if the cosmic ray plasma were at rest with respect to the Galactic centre. This indicates that Galactic cosmic rays co-rotate with the local Galactic magnetic field. Also smaller structures on scales between $15^{\circ}$ and $30^{\circ}$ are visible. The relative amplitude of the smaller structures is about a 


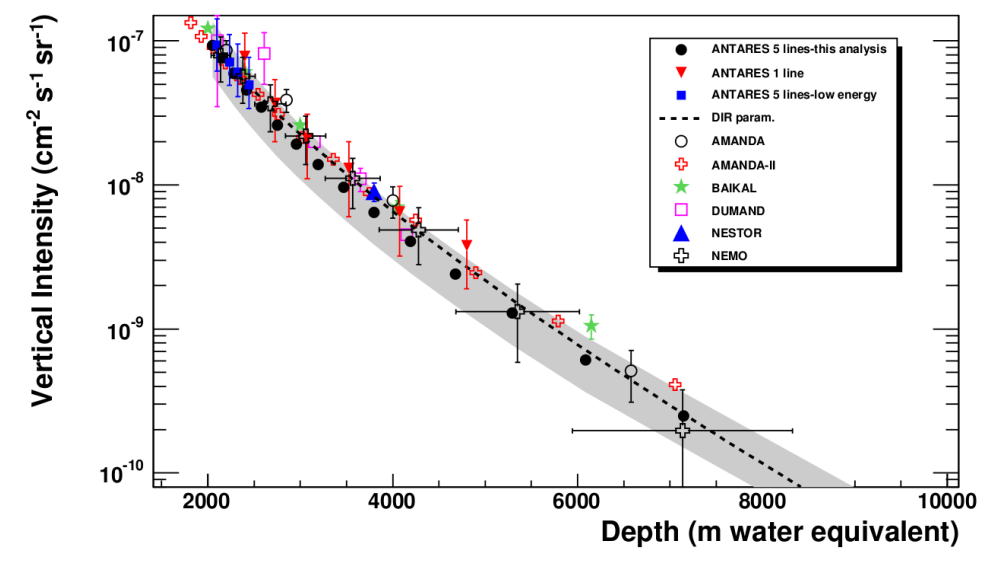

Figure 45. Depth-intensity relation for muons measured by various detectors underground and underwater/ice. The shaded band indicates the systematic uncertainty of the ANTARES analysis, the dashed curve is the expectation derived from [262]. Note that single experiments can cover extended depth ranges by investigating muons incident under different zenith angles. Figure taken from [261].

factor of five weaker than that of the dipole/quadrupole structure. The minima and maxima on all scales are reproducible in all hitherto analysed data samples, from IceCube-22 [268] to IceCube-59 [269].

Figure 46 shows the combined Milagro/IceCube significance sky-map after applying a band-pass filter to remove small-scale fluctuations $\left(10^{\circ}\right.$ smoothing) and the dipole and quadrupole terms.

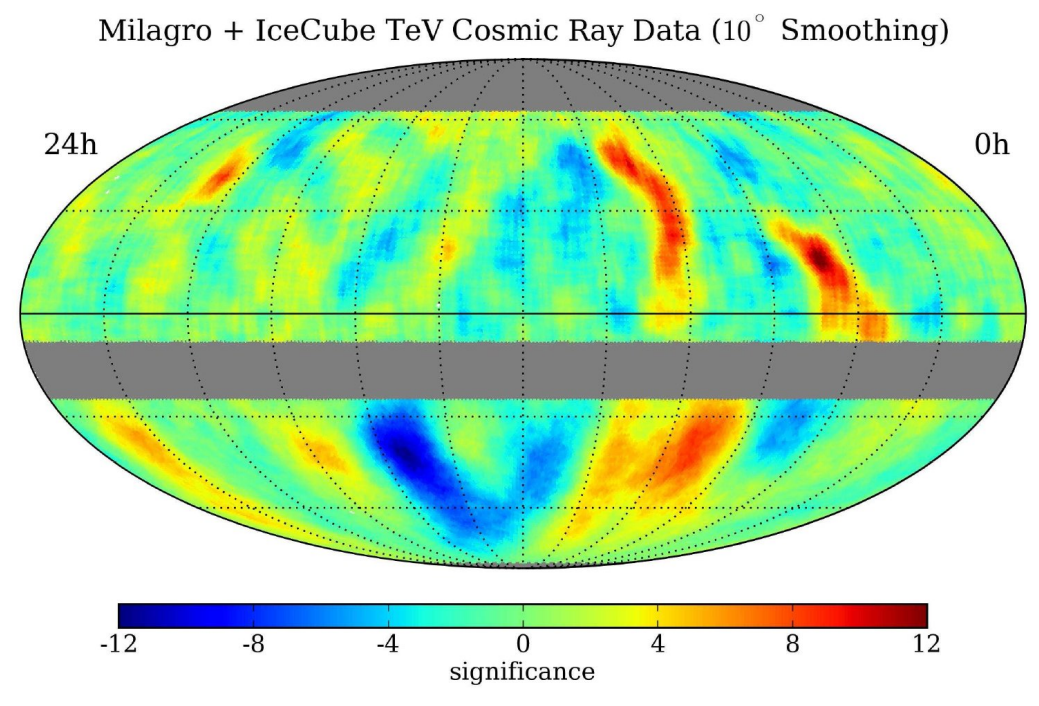

Figure 46. Significance skymap of the medium-scale anisotropies of atmospheric muon arrival directions in IceCube, combined with the Milagro cosmic-ray sky-map for the Northern hemisphere. The colour code indicates the significance of deviations from the average in $\sigma$. Note that structures at very small and very large scales have been filtered out (see text). Figure taken from [269].

The Milagro data contain $2.2 \times 10^{11}$ air showers with a median energy of about $1 \mathrm{TeV}$, the overall IceCube data contain $3.4 \times 10^{10}$ muons with a median energy of the primary particle of about $20 \mathrm{TeV}$. The origin of the medium scale anisotropies is still unknown. Possible explanations include a magnetic "nozzle" to a nearby supernova remnant, with a scatter-free transport along a field connected to the source [270]; at lower energies and small scales, the heliotail may play a role [271]. What ever the origin of the anisotropies is: These structures observed with IceCube may contain key information on nearby sources and/or cosmic ray propagation. To understand the anisotropies is an interesting challenge that will be re-addressed with the increasing statistics expected in the coming years. 


\section{Alternative Detection Principles for Extreme Energies}

The alternative detection technologies described in this section are tailored to signals which propagate with kilometre-scale attenuation. Consequently, they allow for the observation of much larger volumes than those achievable for optical neutrino telescopes. Detectors on the 100 to $1000 \mathrm{~km}^{3}$ scale are necessary, for instance, to record more than just a few cosmogenic neutrinos, in the typical energy range of $100 \mathrm{PeV}$ to $10 \mathrm{EeV}$. This section is intended to give an outlook to future experimental opportunities. Some results of initial projects are included in Sect. 6, see in particular Fig. 36.

\subsection{Detection via air showers}

At energies above $10^{17} \mathrm{eV}$, large air shower arrays like the Pierre Auger Observatory (PAO) in Argentina [272] or the Telescope Array in Utah, USA [273] are seeking for horizontal air showers induced by neutrino interactions deep in the atmosphere (showers caused by charged cosmic ray interactions start much higher up in the atmosphere). Figure 47 explains the detection principle. PAO consists of an array of water tanks covering an area of $3000 \mathrm{~km}^{2}$ that record the Cherenkov light of charged air-shower particles crossing the tanks. The array is combined with telescopes recording the atmospheric fluorescence light from air showers. The optimum sensitivity window for this method is at $1-100 \mathrm{EeV}$, the effective target mass is up to 20 Gigatons. An even better sensitivity might be obtained for tau neutrinos, $\nu_{\tau}$, scratching the Earth and interacting close to the array [274, 275]. The charged $\tau$ lepton produced in charged-current interaction can escape the rock around the array (in contrast to electrons) and mostly decays into hadrons (branching ratio ca. 65\%) after a short path length (in contrast to muons). If this decay happens in the field of view of the fluorescence telescopes, the decay cascade can be recorded. Provided the experimental pattern allows for a clear identification, the acceptance for this kind of signals can be large. For the optimal energy scale of $1 \mathrm{EeV}$, the present $\nu_{\tau}$ limit for an $E^{-2}$ tau neutrino flux is about $E^{2} \phi<10^{-7} \mathrm{GeV} \mathrm{cm}^{-2} \mathrm{~s}^{-1} \mathrm{sr}^{-1}$ [276].

Space-based observation of extended air showers is an approach to even further increase the target mass for highest-energy cosmic-ray and neutrino detection, at energies beyond $10^{19} \mathrm{eV}$. The "Extreme Universe Space Observatory (EUSO)", proposed around the year 2000 , is a wide-field camera observing the atmosphere from an orbit at several $100 \mathrm{~km}$ height and registering the fluorescence light from extended air showers and, if circumstances allow, also the reflection at Earth surface of the Cherenkov light emitted in shower direction [277]. After several years of technical development and a long phase of uncertainty concerning the space carrier, plans are now to install the device - meanwhile renamed to JEM-EUSO [278] - on the Japanese Experiment Module (JEM) of the International Space Station, with the launch expected around 2015. JEM-EUSO will observe an atmospheric target volume with a mass of more than one Teraton and will thus exceed the PAO sensitivity by two orders of magnitude for energies above some $10^{19} \mathrm{eV}$ [279]. Similarly as for PAO, neutrino-induced air showers can be separated from those from hadrons or gammas by the depth of the interaction point in the atmosphere. The JEM-EUSO physics opportunities in the neutrino channel have been studied in general [280, 281] and specifically for cosmogenic neutrinos 


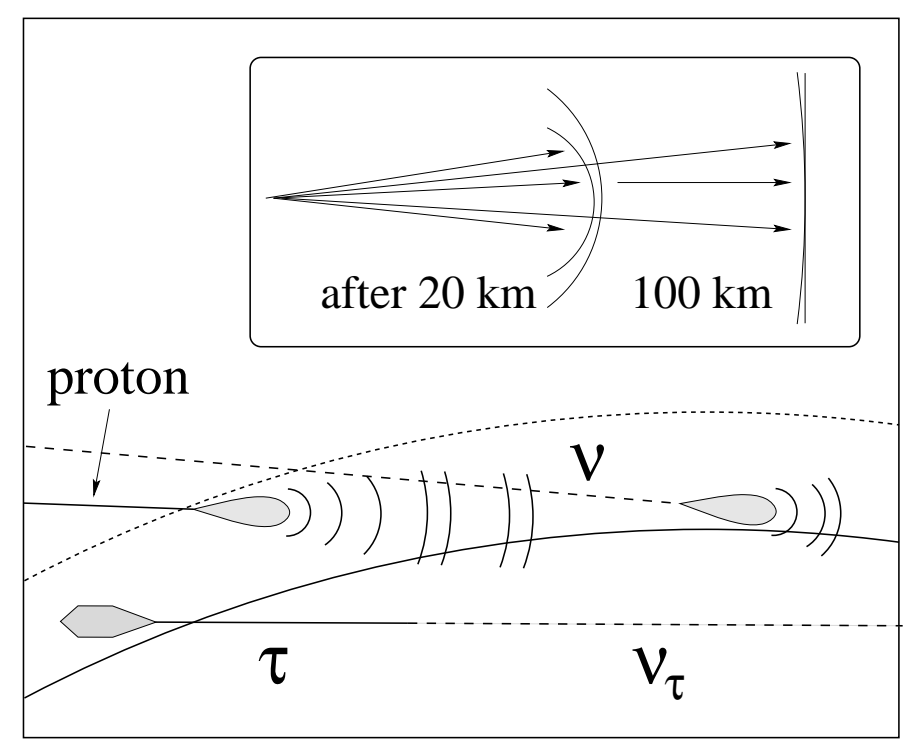

Figure 47. Detection of particles or fluorescence light emitted by horizontal or upwards-directed air showers from neutrino interactions.

[282 and GRB neutrinos [283]. It is not obvious that JEM-EUSO will detect neutrinos from known astrophysical or cosmogenic sources, but its measurements will explore possible neutrino fluxes in a hitherto inaccessible energy region.

\subsection{Radio detection}

Electromagnetic cascades, e.g. generated by high energy neutrino interactions in ice or salt, emit coherent Cherenkov radiation at radio frequencies. The effect was predicted in 1962 284 and confirmed by measurements at accelerators [285, 286]. Electrons from the material traversed are swept into the developing shower, which thus acquires an electric net charge. This charge propagates like a relativistic pancake of about $1 \mathrm{~cm}$ thickness and $10 \mathrm{~cm}$ diameter. For wavelengths exceeding the cascade diameter, coherent emission of electromagnetic radiation (Cherenkov radiation and synchrotron radiation caused by the Earth magnetic field) occurs. The signal amplitude increases with the square of the net charge in the cascade, i.e. it is proportional to $E_{\nu}^{2}$, thus making the method particularly attractive for high-energy cascades. The resulting bipolar pulse is in the radio frequency band and has a width of $1-2$ ns. In ice, attenuation lengths of more than a kilometre are observed for radio signals, depending on the frequency band and the ice temperature, implying that for energies above a few $10 \mathrm{PeV}$ radio detection becomes competitive or superior to optical detection (with its attenuation length of the order $100 \mathrm{~m}$ ) [287].

The prototype "Radio Ice Cerenkov Experiment (RICE)" was operated at the South Pole, with 20 receivers and emitters buried at depths between 120 and $300 \mathrm{~m}$. From the non-observation of very large pulses, limits on the diffuse flux of neutrinos with $E>100 \mathrm{PeV}$ and on the flux of relativistic magnetic monopoles have been derived [288].

The "Antarctic Impulsive Transient Array (ANITA)" [289] is an array of radio antennas which has been flown at a balloon on an Antarctic circumpolar path in 2006 and 2008/09 (see Fig. 48, left). From $35 \mathrm{~km}$ altitude it searched for radio pulses from neutrino interactions in the thick ice cover and monitored, with a threshold in the $\mathrm{EeV}$ range, a volume of the 
order of $10^{6}$ Gigatons. The resulting neutrino flux limits [206] are presented in Fig. 36 in Sect. 6.2.1.
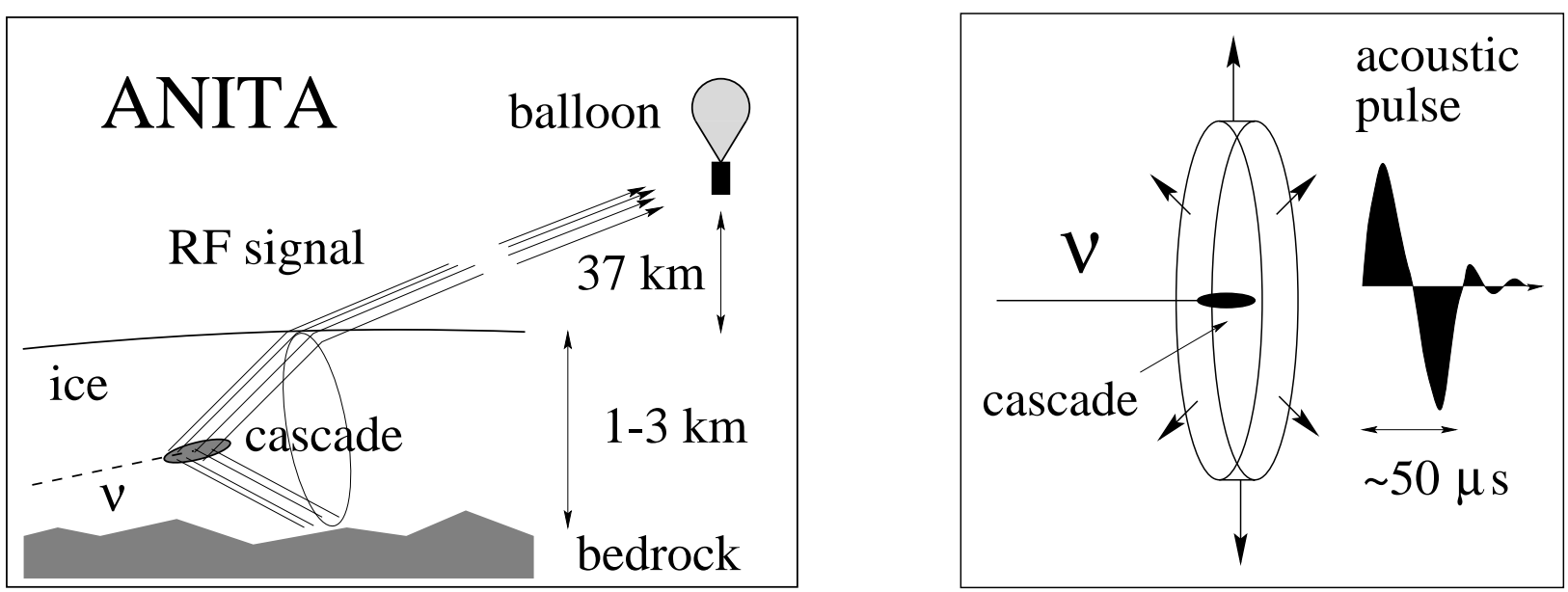

Figure 48. Principles of radio and acoustic detection of neutrinos. Left: ANITA balloon experiment. Right: Acoustic emission of a particle cascade.

Future plans for radio detection in ice foresee large arrays of antennas on the surface of the antarctic ice shelf (ARIANNA [290]) or in the South Polar ice close to the surface (ARA [291]).

Even more exotic is the search for radio emission from extremely-high energy cascades induced by neutrinos or cosmic rays skimming the moon surface. An example is the "Goldstone Ultra-high Energy Neutrino Experiment (GLUE)" which used two NASA antennas and reached a maximum sensitivity at several $\mathrm{ZeV}=1000 \mathrm{EeV}$ [292]. With the same method, the NuMoon experiment at the Westerbork Radio Telescope was searching for extremely energetic neutrinos [293]. Similar activities are under preparation in the context of the LOFAR experiment [294].

\subsection{Acoustic detection}

Production of pressure waves by charged particles depositing energy in liquids or solid media was predicted in 1957 [295] and experimentally proven with high-intensity proton beams two decades later [296]. In the case of a particle cascade, its entire energy is deposited into the medium, mostly through ionisation, and converted to heat on a time scale that is very short compared to the typical time scales relevant for generation and propagation of acoustic pulses. The effect is a fast expansion, generating a bipolar acoustic pulse with a width of a few ten microseconds in water or ice (see Fig. 48, right), corresponding to a peak signal power at $20 \mathrm{kHz}$. Transversely to the pencil-like cascade, the acoustic pulse propagates into the medium within a disk-shaped volume with a thickness corresponding to the cascade length of about $10 \mathrm{~m}$. Exploiting this method would require to detect the acoustic pulses on the background of the ambient and intrinsic noise with a sparsely instrumented detector. This implies a very high detection threshold, in the EeV range at best. 
Acoustic detection is an option both for ice and sea water. For ice, the signal itself is expected to be higher and ambient noise to be lower than in sea water. A test array, SPATS (South Pole Acoustic Test Setup), has been deployed at the South Pole in order to determine the depth dependence of the speed of sound [297], the attenuation length of acoustic signals [298] and the ambient noise [299]. The results of the latter two measurements are slightly discouraging as the attenuation length turns out to be about $300 \mathrm{~m}$, an order of magnitude smaller than expected, and the noise level is roughly the same as in the deep sea at a calm sea state. As a variation of the ice approach, even the use of permafrost as medium has been discussed [300].

Test of acoustic detection in sea water have been performed close to Sicily ( $\nu \nu \mathrm{DE}$ setup, see Sect. 4.5), close to Scotland and in Lake Baikal (see [301 303] for overviews). Another project, SAUND, has been using a very large but extremely sparsely instrumented hydrophone array of the US Navy, close to the Bahamas 304 306. The array of hydrophones covers an area of $250 \mathrm{~km}^{2}$, has good sensitivity at $1-500 \mathrm{kHz}$ and can trigger on events above $100 \mathrm{EeV}$ with a tolerable background rate.

An extended test configuration with various hydrophones, named AMADEUS, has been deployed together with the ANTARES detector [307]. The sensors are arranged in local clusters (size scale one metre), with inter-cluster distances between $12.5 \mathrm{~m}$ and $340 \mathrm{~m}$. First studies focussed on the ambient noise levels (see Fig 49) and their dependence on sea state and precipitation, with the conclusion that under calm conditions the noise in the relevant frequency range is below typical acoustic neutrino signals. In addition, the 3-dimensional arrangement of hydrophones allows for locating transient signals and investigating the rate of background signals of the expected bipolar shape that come from an appropriately defined fiducial volume. For the first time in the deep sea, the intrinsic background to acoustic neutrino signals can thus be studied down to an amplitude level of a few 10 mbar, corresponding to detection thresholds at the EeV scale.

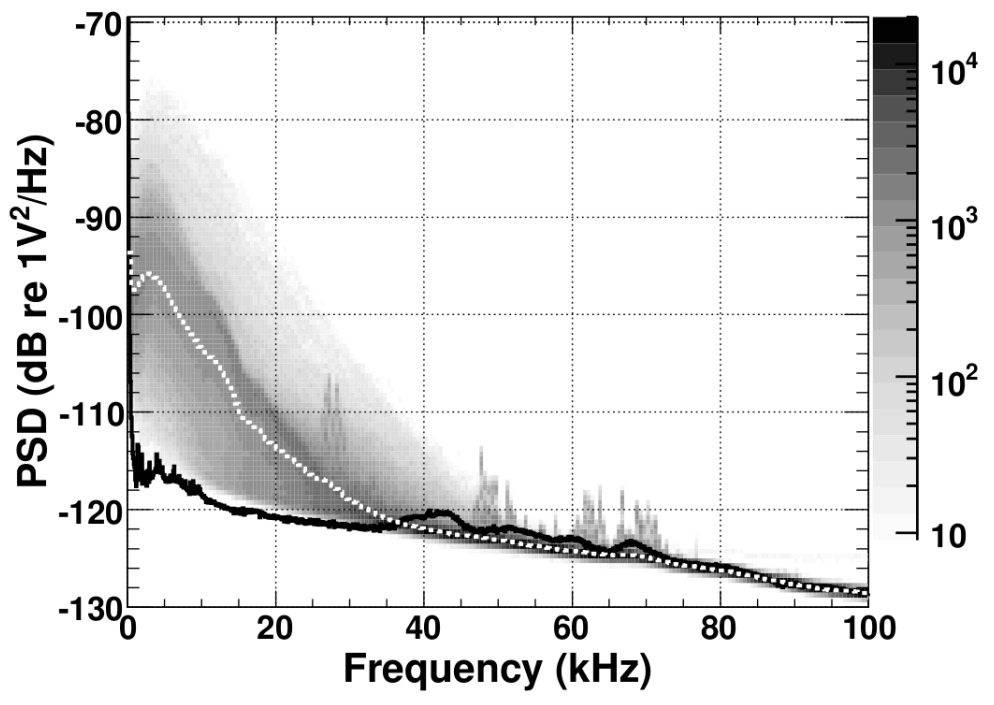

Figure 49. Power spectral density (PSD) of the ambient noise recorded in AMADEUS. Shown in shades of grey is the occurrence rate in arbitrary units, where dark colours indicate higher rates. The white dotted line is the median value of the in-situ PSD and the black solid line indicates the noise level recorded in the laboratory prior to deployment. 


\section{Summary and Outlook}

Five decades after first proposals, and three decades after first practical attempts to build high-energy neutrino telescopes, we may be close to a turning point. IceCube, the first cubic-kilometre neutrino telescope, has started data taking in its final configuration, and preparations for counterparts on the Northern hemisphere are advanced.

The strong case for high-energy neutrino astronomy has remained unchanged over all the time, but the requirements on the necessary sensitivity have tightened continuously. The detection of first high-energy neutrino sources from outer space lays still ahead and may, with some optimism, be expected in the next few years. Galactic "Pevatrons" as those detected by Milagro are in reach after a few years of IceCube data taking if the corresponding predictions are correct. Models assigning the most energetic cosmic rays to Gamma Ray Bursts will be tested within a couple of years. However, clear observations are all but guaranteed.

Discoveries or non-discoveries of IceCube will have a strong impact on the future of the field and possibly mark a "moment of truth". Clear evidence for neutrino sources would pave the straight way for KM3NeT in the Mediterranean Sea, with possible modifications to the present design (high energies vs. low energies) according to the IceCube results. KM3NeT would then complement IceCube in its field of view; for results not depending on the hemisphere (e.g. diffuse fluxes, exotic particles) it could confirm and extend the IceCube findings using a detector with different systematic uncertainties, and in addition it would explore the the central parts of the Galactic plane with superior sensitivity.

Missing or marginal evidence for sources from IceCube may lead to different developments. One option would be to continue the venue of detectors which explore the energy range characteristic for Gamma Ray Bursts and Active Galactic Nuclei. This would require configurations with significantly larger spacing than the present KM3NeT design, resulting in a penalty at energies below a few tens of $\mathrm{TeV}$, and in sacrificing many Galactic source candidates. Abundance and characteristics of extragalactic sources are not expected to differ much between the Northern and Southern skies. A factor of three or so in sensitivity compared to IceCube would therefore only provide a limited additional discovery window for extragalactic sources. Since firm flux predictions are absent, the resulting "gambling" would be justified only if indeed a substantially larger sensitivity increase with respect to IceCube could be reached.

Predictions for Galactic sources are much more firm, provided we assume dominantly hadronic emission. In contrast to extragalactic sources, we have good reasons to assume that we may be close to the discovery region, so that a sensitivity increase by a factor $3-5$, with a telescope observing the Southern hemisphere, indeed counts. Therefore a second option would be to focus on Galactic sources like Supernova Remnants and tailor the array to these sources, i.e. keeping high sensitivity down to about a $\mathrm{TeV}$. The uncertainty here comes from the question to what extent the observed gamma rays are indeed due to hadronic production.

The third option would be an even larger leap in size than for the first option. It would address energies above $100 \mathrm{PeV}$ with the help of new technologies like radio or acoustic detection and envisage 100-1000 cubic kilometres of instrumented volume. This option 
might still have sensitivity to neutrinos from AGN jets but would also well cover the energy range of cosmogenic neutrinos. In contrast to optical detectors, new-technology detectors are still in the R\&D stage and also have no natural calibration source like atmospheric neutrinos for optical detectors.

The fourth option would define, at least for the time being, an end to the search for neutrinos from cosmic accelerators. The field would focus on optical neutrino detection with dense detectors optimised for investigating oscillations of accelerator neutrinos (Mediterranean Sea) and atmospheric neutrinos, or, even more pretentious, to study supernova bursts beyond our own Galaxy or even proton decay.

For the moment, however, we don't see a reason to be pessimistic. We have made a factor-of-thousand step in sensitivity compared to a dozen years ago. This is far more than the traditional factor of ten which so often led to the discovery of new phenomena. For instance, looking back to our own field, the prospects for discovery had not been estimated overly high before launching the first X-ray rocket in 1962, or before detecting the Crab in $\mathrm{TeV}$ gamma rays in 1989. History told a different story, as we know by today.

We have a good chance to open a new window to the Universe, but we don't know it for sure - and that, at the end, may be the most tempting and challenging situation that we possibly can imagine!

Acknowledgements: The authors wish to thank their colleagues in the ANTARES, Baikal, IceCube and KM3NeT collaborations for valuable input and in particular Jürgen Brunner and Alexander Kappes for carefully reading the manuscript and for many important remarks and corrections. 


\section{References}

[1] K. Greisen, Cosmic ray showers, Ann. Rev. Nucl. Part. Sci. 10 (1960) 63.

[2] F. Reines, Neutrino interactions, Ann. Rev. Nucl. Part. Sci. 10 (1960) 1.

[3] M.A. Markov, On high energy neutrino physics, in: Proc. 10th ICHEP, Rochester, 1960, p. 578.

[4] V.S. Berezinsky and G.T.Zatsepin, Cosmic rays at ultra high energies (neutrino?), Phys. Lett. B 28 (1969) 423.

[5] T.K. Gaisser, F. Halzen and T.Stanev, Particle astrophysics with high-energy neutrinos, Phys. Rep. 258 (1995) 173. arXiv:hep-ph/9410384.

[6] J.G. Learned and K. Mannheim, High-energy neutrino astrophysics, Ann. Rev. Nucl. Part. Sci. 50 (2000) 679 .

[7] A.B. McDonald et al., Astrophysical neutrino telescopes, Rev. Sci. Instrum. 75 (2004) 293. arXiv:astro-ph/0311343.

[8] J.K. Becker, High-energy neutrinos in the context of multimessenger physics, Phys. Rep. 458 (2008) 173. arXiv:0710.1557 [astro-ph].

[9] T. Chiarusi and M.Spurio, High-energy astrophysics with neutrino telescopes, Eur. Phys. J. C 65 (2010) 649. arXiv:0906.2634 [astro-ph.HE].

[10] L.A. Anchordoqui and T. Montaruli, In search for extraterrestrial high energy neutrinos, Ann. Rev. Nucl. Part. Sci. 60 (2010) 129. arXiv:0912.1035 [astro-ph.HE]

[11] Fréjus Coll., W. Rhode et al., Limits on the flux of very high-energetic neutrinos with the Fréjus detector, Astropart. Phys. 4 (1996) 217.

[12] M. Harwit, Cosmic discovery, Basic Books Inc., New York, 1981.

[13] F. Halzen. Ice fishing for neutrinos [online] (1995). Available from: http://icecube.berkeley.edu/amanda/ice-fishing.html.

[14] J. Blümer, R. Engel and J.R. Hörandel, Cosmic rays from the knee to the highest energies, Prog. Part. Nucl. Phys. 63 (2009) 293.

[15] E. Fermi, On the origin of the cosmic radiation, Phys. Rev. 75 (1949) 1169.

[16] E. Fermi, Galactic magnetic fields and the origin of cosmic radiation, Astrophys. J. 119 (1954) 1.

[17] V.L. Ginzburg and V.S. Ptuskin, On the orgin of cosmic rays: Some problems in high-energy astrophysics, Rev. Mod. Phys. 48 (1976) 161.

[18] R. Blandford and D. Eichler, Particle acceleration at astrophysical shocks: A theory of cosmic ray orgin, Phys. Rep. 154 (1987) 1.

[19] A.M. Hillas, Can diffuse shock acceleration in supernova remnants account for high-energy galactic cosmic rays?, J. Phys. G 31 (2005) R95.

[20] D. Caprioli, P. Blasi, E. Amato and M. Vietri, Dynamical feedback of self-generated magnetic fields in cosmic rays modified shocks, Mon. Not. Roy. Astron. Soc. 395 (2009) 895.

[21] T. Gaisser, Cosmic rays and particle physics, Cambridge University Press, Cambridge, UK, 1991.

[22] W. Baade and F. Zwicky, Remarks on super-novae and cosmic rays, Phys. Rev. 46 (1934) 76.

[23] I.F. Mirabel, Gamma-ray binaries, Astrophys. Space Sci. 309 (2007) 267. arXiv:astro-ph/0610707.

[24] Pierre Auger Coll., P. Abreu et al., The Pierre Auger Observatory I: The cosmic ray energy spectrum and related measurements, contribution to 32nd Int. Cosmic Ray Conf., Beijing, China (2011). arXiv: 1107.4809.

[25] K. Greisen, End to the cosmic ray spectrum?, Phys. Rev. Lett. 16 (1966) 748.

[26] G.T.Zatsepin and V.A. Kuzmin, Upper limit of the spectrum of cosmic rays, JETP Lett. 4 (1966) 78.

[27] T.K. Gaisser, Neutrino astronomy: Physics goals, detector parameters, OECD Megascience Forum, Taormina, Italy (1997). arXiv:astro-ph/9707283.

[28] R.A. Ong, The status of VHE gamma-ray astronomy, rapporteur Talk at 29th Int. Cosmic Ray Conf., Pune, India (2005). arXiv:astro-ph/0605191.

[29] J.Hinton, Ground based gamma-ray astronomy with Cherenkov Telescopes, New J. Phys. 11 (2009) 055005. arXiv:0803.1609 [astro-ph].

[30] H.E.S.S. Coll., F.A. Aharonian et al., High-energy particle acceleration in the shell of a supernova remnant, Nature 432 (2004) 75. 
[31] Fermi LAT Coll., A.A. Abdo et al., Observations of the young supernova remnant RX J1713.7-3946 with the Fermi Large Area Telescope, Astrophys. J. 734 (2011) 28. arXiv:1103.5727 [astro-ph.HE].

[32] D.C. Ellison, D.J. Patnaude, P. Slane and J. Raymond, Efficient cosmic ray acceleration, hydrodynamics, and self-consistent thermal X-ray emission applied to SNR RX J1713.7-3946, Astrophys. J. 712 (2010) 287. arXiv:1001.1932 [astro-ph.HE].

[33] H.E.S.S. Coll., F.A. Aharonian et al., Detection of TeV $\gamma$-ray emission from the shell-type supernova remnant RX J0852.0-4622 with HESS, Astron. \& Astrophys. L7 (2005) 437. arXiv: astro-ph/0505380.

[34] H.E.S.S. Coll., F.A. Aharonian et al., Discovery of very-high-energy gamma-rays from the Galactic Centre ridge, Nature 439 (2006) 695.

[35] D. Berge, M. Lemoine-Goumard and M. de Naurois for the H.E.S.S. Coll., Observations of SNR RX J1713.7-3946 with H.E.S.S., AIP Conf. Proc. 745 (2005) 223, contribution to 3rd Int. Symp. High-Energy Gamma-Ray Astron., Heidelberg, 2004.

[36] A. Kappes et al., Potential neutrino signals from Galactic gamma-ray sources, Astrophys. J. 656 (2007) 870. arXiv:astro-ph/0607286.

[37] A.A. Abdo et al., Discovery of TeV gamma-ray emission from the Cygnus region of the Galaxy, Astrophys. J. 658 (2007) L33. arXiv:astro-ph/0611691.

[38] A.A. Abdo et al., A measurement of the spatial distribution of diffuse TeV gamma ray emission from the Galactic Plane with Milagro, Astrophys. J. 688 (2008) 1078. arXiv:0805.0417 [astro-ph].

[39] F. Halzen, A. Kappes and A. O'Murchadha, Prospects for identifying the sources of the Galactic cosmic rays with IceCube, Phys. Rev. D 78 (2008) 063004. arXiv:0803.0314 [astro-ph].

[40] F.A. Aharonian et al., Microquasar LS 5039: A TeV gamma-ray emitter and a potential TeV neutrino source, J. Phys. Conf. Ser. 39 (2007) 408. arXiv: astro-ph/0508658.

[41] P. Lipari, Perspectives of high energy neutrino astronomy, Nucl. Inst. Meth. A 567 (2006) 405. arXiv: astro-ph/0605535.

[42] W. Bednarek, T.F. Burgio and T. Montaruli, Galactic discrete sources of high energy neutrinos, New Astron. Rev. 49 (2005) 1. arXiv:astro-ph/0404534

[43] F. Vissani, Neutrinos from galactic sources of cosmic rays with known gamma-ray spectra, Astropart. Phys. 26 (2006) 310. arXiv:astro-ph/0607249.

[44] NEMO Coll., S. Aiello et al., Sensitivity of an underwater Črenkov $\mathrm{km}^{3}$ telescope to TeV neutrinos from Galactic Microquasars, Astropart. Phys. 28 (2007) 1. arXiv:astro-ph/0608053.

[45] F. Vissani, F. Aharonian and N. Sahakyan, On the detectability of high-energy Galactic neutrino sources, Astropart. Phys. 34 (2011) 778. arXiv:1101.4842 [astro-ph.HE].

[46] Fermi LAT Coll., A.A. Abdo et al., Fermi Large Area Telescope bright gamma-ray source list, Astrophys. J. Suppl. 183 (2009) 46. arXiv:0902.1340 [astro-ph]

[47] F.A. Aharonian, J. Buckley, T. Kifune and G. Sinnis, High energy astrophysics with ground-based gamma ray detectors, Rep. Prog. Phys. 71 (2008) 096901.

[48] W. Benbow et al., for the H.E.S.S. Coll., A spectcular VHE gamma-ray outburst from PKS 2155-304 in 2006, in: R. Caballero et al. (Ed.), Proc. 30th Int. Cosmic Ray Conf., Merida, Mexico, Vol. 3, 2007, p. 1081. arXiv:0709.4608 [astro-ph].

[49] H. Krawczynski et al., Multiwavelength observations of strong flares from the TeV-blazar $1 E S 1959+650$, Astrophys. J. 601 (2004) 151. arXiv: astro-ph/0310158.

[50] E. Waxman and J.N. Bahcall, High energy neutrinos from cosmological gamma-ray burst fireballs, Phys. Rev. Lett. 78 (1997) 2292. arXiv:astro-ph/9701231.

[51] K. Razzaque, P. Meszaros and E. Waxman, Neutrino tomography of gamma-ray bursts and massive stellar collapses, Phys. Rev. D 68 (2003) 083001. arXiv:astro-ph/0303505

[52] E. Waxman and J.N. Bahcall, Neutrino afterglow from gamma-ray bursts: $\sim 10^{18} \mathrm{eV}$, Astrophys. J. 541 (2000) 707. arXiv:hep-ph/9909286.

[53] H.E.S.S. Coll., F. Acero et al., Detection of gamma rays from a starburst galaxy, Science 326 (2009) 1080. arXiv:0909.4651 [astro-ph.HE].

[54] N. Karlsson for the VERITAS Coll., Discovery of VHE gamma-ray emission from the starburst 
galaxy M82, presented at FERMI symposium, Washington D.C., USA (2009). arXiv:0912.3807 [astro-ph.HE].

[55] A. Loeb and E. Waxman, The cumulative background of high energy neutrinos from starburst galaxies, J. Cosm. Astropart. Phys. 0605 (2006) 003. arXiv:astro-ph/0601695

[56] F.W.Stecker and M.H.Salamon, High-energy neutrinos from quasars, Space Sci. Rev. 75 (1996) 341. arXiv:astro-ph/9501064.

[57] F.W.Stecker, A note on high energy neutrinos from AGN cores, Phys. Rev. D 72 (2005) 107301. arXiv:astro-ph/0510537.

[58] IceCube Coll., R. Abbasi et al., The energy spectrum of atmospheric neutrinos between 2 and $200 \mathrm{TeV}$ with the AMANDA-II detector, Astropart. Phys. 34 (2010) 48. arXiv:1004.2357 [astro-ph.HE].

[59] L.V. Volkova, Energy spectra and angular distributions of atmospheric neutrinos, Sov. J. Nucl. Phys. 31 (1980) 784.

[60] E. Waxman and J. Bahcall, High-energy neutrinos from astrophysical sources: An upper bound, Phys. Rev. D 59 (1999) 023002. arXiv:hep-ph/9807282.

[61] K. Mannheim, R.J. Protheroe and J.P. Rachen, On the cosmic ray bound for models of extragalactic neutrino production, Phys. Rev. D 63 (2001) 023003. arXiv:astro-ph/9812398.

[62] EGRET Coll., P. Sreekumar et al., EGRET observations of the extragalactic gamma-ray emission, Astrophys. J. 494 (1998) 523. arXiv:astro-ph/9709257.

[63] Fermi-LAT Coll. A. Abdo et al., The spectrum of the isotropic diffuse gamma-ray emission derived from first-year Fermi Large Area Telescope data, Phys. Rev. Lett. 104 (2010) 101101. arXiv: 1002.3603 [astro-ph.HE]

[64] V. Berezinsky, A. Gazizov, M. Kachelriess and S.Ostapchenko, Restricting UHECRs and cosmogenic neutrinos with Fermi-LAT, Phys. Lett. B 695 (2011) 13. arXiv:1003.1496[astro-ph.HE].

[65] V.S. Berezinsky and A.Y.Smirnov, Cosmic neutrinos of ultra-high energies and detection possibility, Astrophys. Space Sci. 32 (1975) 461.

[66] C. Barbot, M. Drees, F. Halzen and D. Hooper, Neutrinos associated with cosmic rays of top-down origin, Phys. Lett. B 555 (2003) 22. arXiv:hep-ph/0205230

[67] Pierre Auger Coll., J. Abraham et al., Upper limit on the cosmic-ray photon flux above $10^{19} \mathrm{eV}$ using the surface detector of the Pierre Auger Observatory, Astropart. Phys. 29 (2008) 243. arXiv:0712.1147 [astro-ph].

[68] A. Silvestri, Limit on ultra high energy neutrino flux, Ph.D. thesis, University of California, Irvine, ISBN 978-0-549-48228-4 (2007). Available from: http://www.ps.uci.edu/tilsilvestr/DISSERTATION/DISSERTATION.pdf

[69] IceCube Coll., J. Ahrens et al., Sensitivity of the IceCube detector to astrophysical sources of high energy muon neutrinos, Astropart. Phys. 20 (2004) 507. arXiv:astro-ph/0305196.

[70] R.C. Cotta, J.S. Gainer, J.L. Hewett and T.G. Rizzo, Dark matter in the MSSM, New J. Phys. 11 (2009) 105026. arXiv:0903.4409[hep-ph].

[71] R. Gilmore, Mass limits on neutralino dark matter, Phys. Rev. D 76 (2007) 043520. arXiv:0705.2610[hep-ph].

[72] K. Olive, Dark matter in SuperGUT unification models, J. Phys. Conf. Ser. 315 (2011) 012021. arXiv: 1009.0232[hep-ph].

[73] G. Jungmann, M. Kamionkowski and K. Griest, Supersymmetric dark matter, Phys. Rep. 267 (1996) 195. arXiv:hep-ph/9506380.

[74] F. Halzen and D. Hooper, The indirect search for dark matter with IceCube, New J. Phys. 11 (2009) 105019. arXiv:0910.4513 [astro-ph.HE].

[75] G. Bertone, D. Hooper and J.Silk, Particle dark matter: Evidence, candidates and constraints, Phys. Rep. 405 (2005) 279. arXiv:hep-ph/0404175.

[76] L. Bergström, Dark matter candidates, New J. Phys. 11 (2009) 105006. arXiv:0903.4849 [hep-ph].

[77] J. Ellis, New light on dark matter from the LHC, in: N.S.M. Borštnik, H.B. Nielsen and D. Lukman (Eds.), Proc. 13th Workshop What Comes Beyond the Standard Models (2010), Bled, Slovenia, 2010, p. 33. arXiv:1012.0222[hep-ph]. 
[78] P.A.M. Dirac, Quantized singularities in the electromagnetic field, Proc. Royal Soc. Lond. A 133 (1931) 60.

[79] G. Giacomelli, S. Manzoor, E. Medinaceli and L. Patrizii, Searches for magnetic monopoles, nuclearites and Q-balls, J. Phys. Conf. Ser. 116 (2008) 012005. arXiv:hep-ex/0702050

[80] S.D. Wick, T.W. Kephart, T.J. Weiler and P.L. Biermann, Signatures for a cosmic flux of magnetic monopoles, Astropart. Phys. 18 (2003) 663. arXiv:astro-ph/0001233.

[81] D. Ryu, H. Kang and P.L. Biermann, Cosmic magnetic fields in large scale filaments and sheets, Astron.\& Astrophys. 335 (1998) 19. arXiv:astro-ph/9803275

[82] V.A. Rubakov, Superheavy magnetic monopoles and proton decay, JETP Lett. 33 (1981) 644.

[83] A. De Rujula and S.L. Glashow, Nuclearites: A novel form of cosmic radiation, Nature 312 (1984) 734.

[84] D. Bakari et al., Magnetic monopoles, nuclearites, Q-balls: A qualitative picture. arXiv: hep-ex/0004019.

[85] A. Kusenko, Solitons in the supersymmetric extensions of the standard model, Phys. Lett. B 405 (1997) 108.

[86] M. Fujii and K. Hamaguchi, Non-thermal dark matter via Afflek-Dine baryogenesis and its detection possibility, Phys. Rev. D 66 (2002) 083501. arXiv:hep-ph/0205044

[87] M. Dine and A. Kusenko, The origin of matter-antimatter asymmetry, Rev. Mod. Phys. 76 (2004) 1. arXiv:hep-ph/0303065.

[88] W. Buchmüller, R.D. Peccei and T. Yanagida, Leptogenesis as the origin of matter, Ann. Rev. Nucl. Part. Sci. 55 (2005) 311. arXiv:hep-ph/0502169

[89] L. Wolfenstein, Neutrino oscillations in matter, Phys. Rev. D 17 (1978) 2369.

[90] S.P. Mikheev and A.Y.Smirnov, Resonance amplification of oscillations in matter and spectroscopy of solar neutrinos, Sov. J. Nucl. Phys. 42 (1985) 913.

[91] Particle Data Group, K. Nakamura et al., Review of particle physics, J. Phys. G 37 (2010) 075021.

[92] D. Grant, D.J. Koskinen and C. Rott for the IceCube Coll., Fundamental neutrino measurements with IceCube DeepCore, contribution to 31st Int. Cosmic Ray Conf., Łódź, Poland (2009). Available from: http://icrc2009.uni.lodz.pl/proc/pdf/icrc1336.pdf.

[93] J.J. Learned and S. Pakvasa, Detecting tau-neutrino oscillations at PeV energies, Astropart. Phys. 3 (1995) 267. arXiv:hep-ph/9405296, hep-ph/9408296

[94] J.F. Beacom et al., Measuring flavor ratios of high-energy astrophysical neutrinos, Phys. Rev. D 68 (2003) 093005. arXiv: hep-ph/0307025.

[95] O. Mena, I. Mocioiu and S. Razzaque, Neutrino mass hierarchy extraction using atmospheric neutrinos in ice, Phys. Rev. D 78 (2008) 093003. arXiv:0803.3044 [hep-ph].

[96] E. Fernandez-Martinez, G. Giordano, O. Mena and I. Mocioiu, Atmospheric neutrinos in ice and measurement of neutrino oscillation parameters, Phys. Rev. D 82 (2010) 093011. arXiv: 1008.4783[hep-ph]

[97] S. Coleman and S.L. Glashow, High-energy tests of Lorentz invariance, Phys. Rev. D 59 (1999) 116008. arXiv:hep-ph/9812418.

[98] IceCube Coll., R. Abbasi et al., Determination of the atmospheric neutrino flux and searches for new physics with AMANDA-II, Phys. Rev. D 79 (2009) 102005. arXiv:0902.0675[astro-ph.HE].

[99] D. Morgan, E. Winstanley, J. Brunner and L.F. Thompson, Neutrino telescope modelling of Lorentz invariance violation in oscillations of atmospheric neutrinos, Astropart. Phys. 29 (2008) 345. arXiv:0705.1897 [astro-ph]

[100] P. Favali et al., NEMO-SN-1 the first "real-time" seafloor observatory of ESONET, Nucl. Inst. Meth. A 567 (2006) 462.

[101] NESTOR Coll., G. Aggouras et al., LAERTIS, a multidisciplinary station, Nucl. Inst. Meth. A 567 (2006) 468.

[102] P. Favali, A. de Santis and L. Beranzoli, Sea floor observatories, Springer praxis books in geophysical sciences, Springer, Berlin, 2011, ISBN 978-3-642-11373-4.

[103] KM3NeT Coll., P. Bagley et al., Technical Design Report, ISBN 978-90-6488-033-9 (2010). Available 
from: www.km3net.org.

[104] EMSO homepage. Available from: http://www.emso-eu.org

[105] AMANDA Coll., P. Askebjer et al., Optical properties of the South Pole ice at depths between $0.8 \mathrm{~km}$ and $1 \mathrm{~km}$, Science 267 (1995) 1147.

[106] AMANDA Coll., M. Ackermann et al., Optical properties of deep glacial ice at the South Pole, Journ. Geophys. Res. 111 (2006) D13203.

[107] N.E. Bramall et al., A deep high-resolution optical log of dust, ash, and stratigraphy in South Pole glacial ice, Geophys. Res. Lett. 32 (2005) L21815.

[108] P.B. Price, Microbial life in glacial ice and implications for a cold origin of life, FEMS Microbiol. Ecol. 59 (2007) 217.

[109] U.F. Katz, Deep-Inelastic Positron-Proton Scattering in the High-Momentum-Transfer Regime of HERA, Vol. 168 of Springer Tracts in Modern Physics, Springer, Berlin, Heidelberg, 2000.

[110] S. Forte, Parton distributions at the dawn of the LHC, Acta Phys. Pol. B 41 (2010) 2859. arXiv: 1011.5247 [hep-ph].

[111] A. Connolly, R.S. Thorne and D. Waters, Calculation of high energy neutrino-nucleon cross sections and uncertainties using the MSTW parton distribution functions and implications for future experiments, Phys. Rev. D 83 (2011) 113009. arXiv:1102.0691[hep-ph].

[112] R. Gandhi, C. Quigg, M.H. Reno and I. Sarcevic, Neutrino interactions at ultrahigh energies, Phys. Rev. D 58 (1998) 093009. arXiv:hep-ph/9807264.

[113] A. Cooper-Sarkar and S. Sarkar, Predictions for high energy neutrino cross-sections from the ZEUS global PDF fits 0801 (2008) 075. arXiv:0710.5303 [hep-ph].

[114] ANTARES Coll., E. Aslanides et al., A deep sea telescope for high-energy neutrinos (1999). arXiv:astro-ph/9907432.

[115] I.F.M Albuquerque, J. Lamoureux and G.F. Smoot, Astrophysical neutrino event rates and sensitivity for neutrino telescopes, Astrophys. J. Suppl. 141 (2002) 195. arXiv:hep-ph/0109177.

[116] R. Gandhi, C. Quigg, M.H. Reno and I. Sarcevic, Ultrahigh-energy neutrino interactions, Astropart. Phys. 5 (1996) 81. arXiv:hep-ph/9512364.

[117] C. Spiering, Neutrino detectors under water and ice, in: C.W. Fabjan and H. Schopper (Eds.), Landolt Börnstein, Vol. I/21/B/2/6.2, Springer, Heidelberg, New York, 2011.

[118] Photomultiplier handbook (1989). Available from: http://psec.uchicago.edu/links/Photomultiplier\uscHandbook.pdf.

[119] Hamamatsu, Photomultiplier tubes - basics and applications (2006). Available from: http://sales.hamamatsu.com/assets/applications/ETD/pmt \uschandbook\usccomplete.pdf

[120] D.E. Groom, N.V. Mokhov and S.I. Striganov, Muon stopping power and range tables $10 \mathrm{MeV}-100 \mathrm{TeV}$, Atom. Data Nucl. Data Tabl. 78 (2001) 183.

[121] IceCube Coll., R. Abbasi et al., A search for a diffuse flux of astrophysical muon neutrinos with the IceCube 40-string detector, Phys. Rev. D 84 (2011) 082001. arXiv:1104.5187 [astro-ph.HE].

[122] Whipple Coll., T.C. Weekes et al., Observation of TeV gamma rays from the Crab nebula using the atmospheric Cerenkov imaging technique, Astrophys. J. 342 (1989) 379.

[123] HEGRA Coll., D. Petry et al., Detection of VHE gamma-rays from Mkn-421 with the HEGRA Cherenkov telescopes, Astron. \& Astrophys. 311 (1996) L13. arXiv:astro-ph/9606159.

[124] AMANDA Coll., J. Ahrens et al., Muon track reconstruction and data selection techniques in AMANDA, Nucl. Inst. Meth. A 524 (2004) 169. arXiv:astro-ph/0407044.

[125] ANTARES Coll., J.A. Aguilar et al., A fast algorithm for muon track reconstruction and its application to the ANTARES neutrino telescope, Astropart. Phys. 34 (2011) 652.

[126] Baikal Coll., V.A. Balkanov et al., Registration of atmospheric neutrinos with the BAIKAL neutrino telescope NT-96, Astropart. Phys. 12 (1999) 75. arXiv:astro-ph/9903341

[127] AMANDA Coll., M. Ackermann et al., Search for neutrino-induced cascades with AMANDA, Astropart. Phys. 22 (2004) 127. arXiv:astro-ph/0405218.

[128] B. Hartmann, Reconstruction of neutrino-induced hadronic and electromagnetic showers with the ANTARES experiment, Ph.D. thesis, Univ. Erlangen (2006). arXiv:astro-ph/0606697. 
[129] Baikal Coll., A.V Avrorin et al., Search for high-energy neutrinos in the Baikal neutrino experiment 35 (2009) 651.

[130] A. Roberts, The birth of high-energy neutrino astronomy: A personal history of the DUMAND project, Rev. Mod. Phys. 64 (1992) 259.

[131] DUMAND Coll., P. Bosetti et al., DUMAND II: Proposal to construct a deep-ocean laboratory for the study of high energy neutrino astrophysics and particle physics, Tech. Rep. HDC-2-88, Hawaii DUMAND Center, University of Hawaii (1988).

[132] DUMAND Coll., E. Babson et al., Cosmic ray muons in the deep ocean, Phys. Rev. D 42 (1990) 3613.

[133] Baikal homepage. Available from: http://baikalweb.jinr.ru/

[134] Baikal Coll., I.A. Belolaptikov et al., The Baikal underwater neutrino telescope: Design, performance, and first results, Astropart. Phys. 7 (1997) 263.

[135] Baikal Coll., L.B. Bezrukov et al., Progress report on Lake Baikal neutrino experiment: Site studies and stationary string, in: Proc. XI. Conf. on Neutrino Physics and Astrophysics, Nordkirchen, Germany, 1984, p. 550.

[136] G.V.Domogatsky et al., Present status of Baikal deep underwater experiment, in: Proc. XII. Conf. on Neutrino Physics and Astrophysics, Sendai, Japan, 1986, p. 737.

[137] L.B. Bezrukov et al., Search for superheavy magnetic monopoles in deep underwater experiments at Lake Baikal (in Russian), Sov. J. Nucl. Phys. 52 (1990) 54.

[138] Baikal Coll., R.V. Balkanov et al., Reconstruction of atmospheric neutrinos with the Baikal neutrino telescope NT-96, proc. 25th Int. Cosmic Ray Conf., Durban, South Africa (1997). arXiv:astro-ph/9705244.

[139] R. Bagduev et al., The optical module of the Baikal deep underwater neutrino telescope, Nucl. Inst. Meth. A 420 (1999) 138. arXiv:astro-ph/9903347.

[140] Baikal Coll., V. Aynutdinov et al., Search for a diffuse flux of high-energy extraterrestrial neutrinos with the NT200 neutrino telescope, Astropart. Phys. 25 (2006) 140. arXiv:astro-ph/0508675.

[141] Baikal Coll., V. Aynutdinov et al., The BAIKAL neutrino experiment: From NT200 to NT200+, Nucl. Inst. Meth. A 567 (2006) 433. arXiv:astro-ph/0609743.

[142] AMANDA Coll., E. Andrés et al., The AMANDA neutrino telescope: Principle of operation and first results, Astropart. Phys. 13 (2000) 1. arXiv:astro-ph/9906203.

[143] AMANDA Coll., E. Andrés et al., Observation of high-energy neutrinos using čerenkov detectors embedded deep in Antarctic ice, Nature 410 (2001) 441.

[144] ANTARES homepage. Available from: http://antares.in2p3.fr.

[145] F. Blondeau for the ANTARES Coll., The ANTARES demonstrator: Towards a high-energy undersea neutrino telescope, Prog. Part. Nucl. Phys. 40 (1998) 413.

[146] F. Feinstein for the ANTARES Coll., The ANTARES demonstrator towards an undersea neutrino telescope, Nucl. Phys. Proc. Suppl. 70 (1999) 445.

[147] ANTARES Coll., P. Amram et al., Background light in potential sites for the ANTARES undersea neutrino telescope, Astropart. Phys. 13 (2000) 127.

[148] ANTARES Coll., P. Amram et al., Sedimentation and fouling of optical surfaces at the ANTARES site, Astropart. Phys. 19 (2003) 253. arXiv:astro-ph/0206454.

[149] ANTARES Coll., M. Ageron et al., ANTARES: The first undersea neutrino telescope, Nucl. Inst. Meth. A 656 (2011) 11. arXiv:1104.1607 [astro-ph.IM].

[150] ANTARES Coll., M. Ageron et al., Studies of a full-scale mechanical prototype line for the ANTARES neutrino telescope and tests of a prototype instrument for deep-sea acoustic measurements, Nucl. Inst. Meth. A 581 (2007) 695.

[151] ANTARES Coll., P. Amram et al., The ANTARES optical module, Nucl. Inst. Meth. A 484 (2002) 369. arXiv:astro-ph/0112172

[152] ANTARES Coll., J.A. Aguilar et al., Study of large hemispherical photomultiplier tubes for the ANTARES neutrino telescope, Nucl. Inst. Meth. A 555 (2005) 132. arXiv:physics/0510031[physics.ins-det]

[153] ANTARES Coll., J.A. Aguilar et al., Transmission of light in deep sea water at the site of the 
ANTARES neutrino telescope, Astropart. Phys. 23 (2005) 131. arXiv:astro-ph/0412126.

[154] J. Brunner for the ANTARES Coll., The ANTARES neutrino telescope - status and first results, Nucl. Inst. Meth. A 626-627 (2011) S19.

[155] ANTARES Coll., J.A. Aguilar et al., The data acquisition system for the ANTARES neutrino telescope, Nucl. Inst. Meth. A 570 (2007) 107. arXiv:astro-ph/0610029.

[156] ANTARES Coll., J.A. Aguilar et al., Time calibration of the ANTARES neutrino telescope, Astropart. Phys. 34 (2011) 539. arXiv:1012.2204 [astro-ph.IM]

[157] ANTARES Coll., M. Ageron et al., The ANTARES optical beacon system, Nucl. Inst. Meth. A 578 (2007) 498. arXiv:astro-ph/0703355.

[158] M. Ardid for the ANTARES Coll., Positioning system of the ANTARES neutrino telescope, Nucl. Inst. Meth. A 602 (2009) 174.

[159] A.M. Brown for the ANTARES Coll., Positioning system of the ANTARES neutrino telescope, contribution to 31st Int. Cosmic Ray Conf., Łódź, Poland (2009). Available from: http://icrc2009.uni.lodz.pl/proc/pdf/icrc0178.pdf, arXiv:0908.0814 [astro-ph. IM]

[160] ANTARES Coll., J.A. Aguilar et al., AMADEUS - The acoustic neutrino detection test system of the ANTARES deep-sea neutrino telescope, Nucl. Inst. Meth. A 626-627 (2011) 128. arXiv:1009.4179 [astro-ph.IM].

[161] NEMO homepage. Available from: http://nemoweb.lns.infn.it.

[162] NEMO Coll., E. Migneco et al., NEMO: Status of the project, Nucl. Phys. Proc. Suppl. 136 (2004) 61.

[163] NEMO Coll., E. Migneco et al., Status of NEMO, Nucl. Inst. Meth. A 567 (2006) 444.

[164] NEMO Coll., A. Capone et al., Recent results and perspectives of the NEMO project, Nucl. Inst. Meth. A 602 (2009) 47.

[165] NEMO Coll., S. Aiello et al., Measurement of the atmospheric muon flux with the NEMO Phase-1 detector, Astropart. Phys. 33 (2010) 263. arXiv:0910.1269[astro-ph.IM].

[166] NEMO Coll., M. Taiuti et al., The NEMO project: A status report, Nucl. Inst. Meth. A 626-627 (2011) S25.

[167] NEMO Coll., G. Riccobene et al., Long-term measurements of acoustic background noise in very deep sea, Nucl. Inst. Meth. A 604 (2009) S149.

[168] F. Bénard-Coudal, P. Giraudet and H. Glautin, Whale 3D monitoring using astrophysic NEMO ONDE two meters wide platform with state optimal filtering by Rao-Blackwell Monte Carlo data association, App. Acou. 71 (2010) 994.

[169] NESTOR homepage. Available from: http://www.nestor.noa.gr/.

[170] NESTOR Coll., L.K. Resvanis et al., NESTOR: A neutrino particle astrophysics underwater laboratory for the Mediterranean, Nucl. Phys. Proc. Suppl. 35 (1994) 294.

[171] NESTOR Coll., G. Aggouras et al., Operation and performance of the NESTOR test detector, Nucl. Inst. Meth. A 552 (2005) 420.

[172] NESTOR Coll., G. Aggouras et al., A measurement of the cosmic-ray muon flux with a module of the NESTOR neutrino telescope, Astropart. Phys. 23 (2005) 377.

[173] IceCube homepage. Available from: http://www.icecube.wisc.edu/.

[174] IceCube Coll., J. Ahrens et al., Sensitivity of the IceCube detector to astrophysical sources of high energy muon neutrinos, Astropart. Phys. 20 (2004) 507. arXiv:astro-ph/0305196.

[175] IceCube Collaboration, IceCube Preliminary Design Document (2001). Available from: http://www.icecube.wisc.edu/science/publications/pdd/.

[176] IceCube Coll., R. Abbasi et al., The IceCube data acquisition system: Signal capture, digitization, and timestamping, Nucl. Inst. Meth. A 601 (2009) 294. arXiv:0810.4930[physics.ins-det].

[177] C. Wiebusch for the IceCube Coll., Physics capabilities of the IceCube DeepCore detector, contribution to 31st Int. Cosmic Ray Conf., Łódź, Poland (2009). Available from: http://icrc2009.uni.lodz.pl/proc/pdf/icrc1352.pdf, arXiv:0907.2263[astro-ph. IM]

[178] T. Waldenmaier for the IceCube Coll., IceTop - cosmic ray physics with IceCube, Nucl. Inst. Meth. A 588 (2008) 130. arXiv:0802.2540 [astro-ph].

[179] AMANDA Coll., J. Ahrens et al., Search for supernova neutrino-bursts with the AMANDA detector, 
Astropart. Phys. 16 (2002) 345. arXiv:astro-ph/0105460

[180] T. Kowarik, T. Griesel and A. Piégsa for the IceCube Coll., Supernova search with the AMANDA / IceCube detectors, contribution to 31st Int. Cosmic Ray Conf., Łódź, Poland (2009). Available from: http://icrc2009.uni.lodz.pl/proc/pdf/icrc1251.pdf, arXiv:0908.0441 [astro-ph.HE]

[181] P. Antonioli et al., SNEWS: The supernova early warning system, New J. Phys. 6 (2004) 114. arXiv:astro-ph/0406214.

[182] The High Energy Neutrino Astrophysics Panel, E. Feernandez et al., High energy neutrino observatories (2002). Available from: www.lngs.infn.it/lngs\uscinfn/contents/docs/pdf/panagic/henap2002.pdf.

[183] KM3NeT Coll., P. Bagley et al., Conceptual Design Report, ISBN 978-90-6488-031-5 (2008). Available from: www.km3net.org.

[184] European Strategy Forum on Reasearch Infrastructures (ESFRI), European roadmap for research infrastructures, report 2006 (2006). Available from: ftp://ftp.cordis.europa.eu/pub/esfri/docs/esfri-roadmap-report-26092006/uscen.pdf.

[185] European Strategy Forum on Reasearch Infrastructures (ESFRI), European roadmap for research infrastructures, update 2008 (2008). Available from: ftp://ftp.cordis.europa.eu/pub/esfri/docs/esfri \uscroadmap \uscupdate \usc2008.pdf.

[186] O. Kavatsyuk for the KM3NeT Coll., Photo-sensor characteristics for a multi-PMT optical module in KM3NeT, contribution to 31st Int. Cosmic Ray Conf., Łódź, Poland (2009). Available from: http://icrc2009.uni.lodz.pl/proc/pdf/icrc0767.pdf.

[187] Baikal Coll., V. Aynutdinov et al., The prototype string for the km3-scale Baikal neutrino telescope, Nucl. Inst. Meth. A 602 (2009) 227. arXiv:0811.1110 [astro-ph]

[188] Baikal Coll., A. Avrorin et al., The Baikal experiment - from Megaton to Gigaton, J. Phys. Conf. Ser. 203 (2010) 012123.

[189] S. Gabici et al., The diffuse neutrino flux from the inner Galaxy: Constraints from very high energy gamma-ray observations, Astropart. Phys. 30 (2008) 180. arXiv:0806.2459 [astro-ph].

[190] G.D. Barr, T. Gaisser, S. Robbins and T. Stanev, Uncertainties in atmospheric neutrino fluxes, Phys. Rev. D 74 (2006) 094009. arXiv:astro-ph/0611266

[191] K. Daum et al., Determination of the atmospheric neutrino spectra with the Fréjus detector, Z. Phys. C 66 (1995) 417.

[192] M.C. González-García, M. Maltoni and J. Rojo, Determination of the atmospheric neutrino fluxes from experimental data, Astrophys. Space Sci. 309 (2007) 447.

[193] IceCube Coll., R. Abbasi et al., First search for extremely high energy cosmogenic neutrinos with the IceCube neutrino observatory, Phys. Rev. D 82 (2010) 072003. arXiv: 1009.1442 [astro-ph.C0].

[194] IceCube Coll., R. Abbasi et al., Measurement of the atmospheric neutrino energy spectrum from $100 \mathrm{GeV}$ to $400 \mathrm{TeV}$ with IceCube, Phys. Rev. D 83 (2011) 012001. arXiv: 1010.3980 [astro-ph.HE]

[195] IceCube Coll., R. Abbasi et al., Search for a Lorentz-violating siderial signal with atmospheric neutrinos in IceCube, Phys. Rev. D 82 (2010) 112003. arXiv:1010.4096 [astro-ph.HE].

[196] M. Kowalski, Measuring diffuse neutrino fluxes with IceCube, J. Cosm. Astropart. Phys. 0505 (2005) 010. arXiv:astro-ph/0505506

[197] IceCube Coll., A. Achterberg et al., Multi-year search for a diffuse flux of muon neutrinos with AMANDA-II, Phys. Rev. D 76 (2007) 042008, erratum ibid., D 77 (2008) 089904(E). arXiv:0705.1315 [astro-ph].

[198] ANTARES Coll., J.A. Aguilar et al., Search for a diffuse flux of high-energy $\nu_{\mu}$ with the ANTARES neutrino telescope, Phys. Lett. B 696 (2011) 16. arXiv:1011.3772 [astro-ph.HE].

[199] IceCube Coll., R. Abbasi et al., Search for neutrino-induced cascades with five years of AMANDA data, Astropart. Phys. 34 (2011) 420.

[200] A. Kochanov for the Baikal Coll., Search for a diffuse flux of high-energy neutrinos with the Baikal neutrino telescope NT200, contribution to 31st Int. Cosmic Ray Conf., Łódź, Poland (2009). Available from: http://icrc2009.uni.lodz.pl/proc/pdf/icrc1093.pdf, 
arXiv:0909.5562 [astro-ph.HE].

[201] IceCube Coll., R. Abbasi et al., First search for atmospheric and extraterrestrial neutrino-induced cascades with the IceCube detector (2011). arXiv:1101.1692 [astro-ph.HE].

[202] MACRO Coll., M. Ambrosio et al., Search for diffuse neutrino flux from astrophysical sources with MACRO, Astropart. Phys. 19 (2003) 1. arXiv:astro-ph/0203181.

[203] IceCube Coll., R. Abbasi et al., Constraints on the extremely-high energy cosmic neutrino flux with the IceCube 2008-2009 data, Phys. Rev. D 83 (2011) 092003. arXiv:1103.4250 [astro-ph.CO].

[204] RICE Coll., I. Kravchenko et al., RICE limits on the diffuse ultrahigh energy neutrino flux, Phys. Rev. D 73 (2006) 082002. arXiv:astro-ph/0601148.

[205] P. Billoir for the Pierre Auger Coll., Limit on the diffuse flux of ultra high energy neutrinos using the Pierre Auger Observatory, J. Phys. Conf. Ser. 203 (2010) 012125.

[206] ANITA Coll., P.W. Gorham et al., Observational constraints on the ultrahigh energy cosmic neutrino flux from the second flight of the ANITA experiment, Phys. Rev. D 82 (2010) 022004, Erratum in arXiv:1011.5004 [astro-ph.HE]. arXiv:1003.2961 [astro-ph.HE].

[207] K. Kotera and A.V. Olinto, The astrophysics of ultrahigh energy cosmic rays, Ann. Rev. Astron. Astrophys. 49 (2011) 1. arXiv:1101.4256 [astro-ph.HE].

[208] G.B. Gelmini, O. Kalashev and D.V.Semikoz, Gamma-ray constraints on maximum cosmogenic neutrino fluxes and UHECR source evolution models, to appear in JHEP (2011). arXiv: 1107.1672 [astro-ph.CO].

[209] MACRO Coll., M. Ambrosio et al., Neutrino astronomy with the MACRO detector, Astrophys. J. 546 (2001) 1038. arXiv:astro-ph/0002492.

[210] Super-Kamiokande Coll., E. Thrane et al., Search for astrophysical neutrino point sources at Super-Kamiokande, Astrophys. J. 704 (2009) 503. arXiv:0907.1594 [astro-ph.HE].

[211] IceCube Coll., R. Abbasi et al., Search for point sources of high energy neutrinos with final data from AMANDA-II, Phys. Rev. D 79 (2009) 062001. arXiv:0809.1646 [astro-ph].

[212] J. Braun et al., Methods for point source analysis in high energy neutrino telescopes, Astropart. Phys. 29 (2008) 299. arXiv:0801.1604 [astro-ph]

[213] J. Hernandez, Northern hemisphere neutrino telescopes, presentation at Very Large Volume Neutrino Telescopes (VLVnT11), Erlangen, Germany, Oct. 2011 (2011). Available from: https://indico.cern.ch/contributionDisplay.py?contribId=5 \ampconf Id=143656.

[214] IceCube Coll., R. Abbasi et al., Time-integrated searches for point-like sources of neutrinos with the 40-string IceCube detector, Astrophys. J. 732 (2011) 18. arXiv:1012.2137.

[215] IceCube Coll., R. Abbasi et al., First neutrino point-source results from the 22 string IceCube detector, Astrophys. J. 701 (2009) L47. arXiv:0905.2253 [astro-ph.HE].

[216] IceCube Coll., R. Abbasi et al., Extending the search for neutrino point sources with IceCube above the horizon, Phys. Rev. Lett. 103 (2009) 221102. arXiv:0911.2338[astro-ph.HE]

[217] Amanda Coll., E. Andres et al., Results from the AMANDA high-energy neutrino detector, Nucl. Phys. Proc. Suppl. 91 (2001) 423. arXiv:astro-ph/0009242.

[218] G. Morlino, E. Amato and P. Blasi, Gamma ray emission from SNR RX J1713.7-3946 and the origin of galactic cosmic rays, Mon. Not. Roy. Astron. Soc. 392 (2009) 240. arXiv:0810.0094 [astro-ph].

[219] H.B.J. Koers and P. Tinyakov, Relation between the neutrino flux from Centaurus A and the associated diffuse neutrino flux, Phys. Rev. D 78 (2008) 083009. arXiv:0802.2403 [astro-ph].

[220] J. Braun et al., Time-dependent point source search methods in high energy neutrino astronomy, Astropart. Phys. 33 (2010) 175. arXiv:0912.1572 [astro-ph. IM]

[221] J.L. Bazo Alba, E. Bernardini and R. Lauer for the IceCube Coll., Search for neutrino flares from point sources with IceCube, contribution to 31st Int. Cosmic Ray Conf., Łódź, Poland (2009). Available from: http://icrc2009.uni.lodz.pl/proc/pdf/icrc0960.pdf, arXiv:0908.4209 [astro-ph.HE]

[222] D. Góra, E. Bernardini and A.H. Cruz Silva, A method for untriggered time-dependent searches for multiple flares from neutrino point sources, Astropart. Phys. 35 (2011) 201. arXiv:1103.2644 [astro-ph.IM], 
[223] IceCube Coll., R. Abbasi et al., Time-dependent searches for point sources of neutrinos with the 40-string and 22-string configurations of IceCube (2011). arXiv: 1104.0075 [astro-ph.HE].

[224] M. Ackermann, Searches for signals from cosmic point-like sources of high energy neutrinos in 5 years of AMANDA-II data, Ph.D. thesis, Humboldt University Berlin (2006). Available from: http://edoc.hu-berlin.de/docviews/abstract.php?lang=ger \ampid=27726

[225] E. Bernardini for the IceCube Coll., Multi-messenger studies with AMANDA / IceCube: Observations and strategies, contribution to the Cherenkov 2005 Conference, Palaiseau, France (2005). arXiv: astro-ph/0509396.

[226] A. Reimer, M. Böttcher and S. Postnikov, Neutrino emission in the hadronic synchrotron mirror model: the "orphan" TeV flare from 1ES 1959+650, Astrophys. J. 630 (2005) 186. arXiv: astro-ph/0505233.

[227] F. Halzen and D. Hooper, High energy neutrinos from the TeV blazar 1ES 1959+650, Astropart. Phys. 23 (2005) 537. arXiv:astro-ph/0502449.

[228] IceCube Coll., R. Abbasi et al., Constraints on high-energy neutrino emission from SN 2008D, Astron. \& Astrophys. 527 (2011) A28. arXiv:1101.3942 [astro-ph.HE]

[229] D. Guetta et al., Neutrinos from individual gamma-ray bursts in the BATSE catalog, Astropart. Phys. 20 (2004) 429. arXiv:astro-ph/0302524.

[230] IceCube and the InterPlanetary Network Coll., A. Achterberg et al., The search for muon neutrinos from northern hemisphere gamma-ray bursts with AMANDA, Astrophys. J. 674 (2008) 357. arXiv:0705.1186 [astro-ph].

[231] IceCube Coll., R. Abbasi et al., Limits on neutrino emission from gamma-ray bursts with the 40 string IceCube detector, Phys. Rev. Lett. 106 (2011) 141101. arXiv:1101.1448[astro-ph.HE].

[232] P. Redl for the IceCube Coll., Limits on neutrino emission from gamma-ray bursts with the 59 string IceCube detector, contribution to 32nd Int. Cosmic Ray Conf., Beijing, China (2011). Available from: http://galprop.stanford.edu/elibrary/icrc/2011/papers/HE2.3/icrc0764.pdf.

[233] M. Kowalski and A. Mohr, Detecting neutrino transients with optical follow-up observations, Astropart. Phys. 27 (2007) 533. arXiv: astro-ph/0701618

[234] S. Basa et al., Neutrino alert systems for Gamma Ray Bursts and transient astronomical sources, Nucl. Inst. Meth. A 602 (2009) 275. arXiv:0810.1394 [astro-ph]

[235] ANTARES Coll., M. Ageron et al., The ANTARES telescope neutrino alert system, subm. to Astropart. Phys arXiv:1103.4477 [astro-ph. IM]

[236] IceCube and MAGIC Coll., M. Ackermann et al., Neutrino triggered target of opportunity (NToO) test run with AMANDA-II and MAGIC, in: R. Caballero et al. (Ed.), Proc. 30th Int. Cosmic Ray Conf., Merida, Mexico, Vol. 3, 2008, p. 1257. arXiv:0709.2640 [astro-ph].

[237] Th. Kowarik, T. Griesel and A. Piégsa for the IceCube Coll., Supernova search with the AMANDA / IceCube detectors, contribution to 31st Int. Cosmic Ray Conf., Łódź, Poland (2009). Available from: http://icrc2009.uni.lodz.pl/proc/pdf/icrc1251.pdf, arXiv:0908.0441 [astro-ph.HE]

[238] G. Raffelt, Supernova neutrino observations: What can we learn?, proc. 22nd Int. Conf. Neutrino Physics and Astrophysics, Santa Fe, USA (2007). Available from: http://lss.fnal.gov/conf/C0606131/Neutrino06-Proceedings.pdf, arXiv: astro-ph/0701677

[239] AMANDA Coll., M. Ackermann et al., Limits to the muon flux from neutralino annihilations in the Sun with the AMANDA detector, Astropart. Phys. 24 (2006) 459. arXiv:astro-ph/0508518.

[240] G.M.A. Lim for the ANTARES Coll., First results on the search for dark matter in the Sun with the ANTARES neutrino telescope, contribution to 31st Int. Cosmic Ray Conf., Łódź, Poland (2009). Available from: http://icrc2009.uni.lodz.pl/proc/pdf/icrc0031.pdf.

[241] H. Motz for the ANTARES Coll., Indirect search for dark matter with the ANTARES neutrino telescope, DOI 10.1142/9789814293792_0041, proc. 7th Int. Conf. on Dark Matter in Astrophysics and Particle Physics (Dark09), Christchurch, New Zealand (2009).

[242] G.M.A.Lim, Searching for dark matter with the ANTARES neutrino telescope, Ph.D. thesis, University of Amsterdam, The Netherlands (2011). Available from: 
http://www.nikhef.nl/pub/services/biblio/theses\uscpdf/thesis \uscG \uscLim.pdf

[243] C. Rott for the IceCube Coll., Search for dark matter from the Galactic halo with IceCube (2009). arXiv:0912.5183 [astro-ph.HE].

[244] C. de los Heros for the IceCube Coll., Dark matter searches with IceCube, proc. 8th Int. Workshop on Identification of Dark Matter (IDM2010), Montpellier, France (2010). arXiv:1012.0184.

[245] J. Conrad, Indirect detection of dark matter with gamma-rays - status and perspectives, proc. 8th Int. Workshop on Identification of Dark Matter (IDM2010), Montpellier, France (2011). arXiv:1103.5638 [astro-ph.C0].

[246] T.A. Porter, R.P. Johnson and P.W. Graham, Dark matter searches with astroparticle data, submitted to Ann. Rev. Astron. Astrophys (2011). arXiv:1104.2836 [astro-ph.HE].

[247] R. Kappl and M.W. Winkler, New limits on dark matter from Super-Kamiokande, Nucl. Phys. B 850 (2011) 505. arXiv:1104.0679[hep-ph].

[248] IceCube Coll., R. Abbasi et al., Limits on a muon flux from Kaluza-Klein dark matter annihilations in the Sun from the IceCube 22-string detector, Phys. Rev. D 81 (2010) 057101. arXiv:0910.4480 [astro-ph.C0].

[249] Baikal Coll., V. Aynutdinov et al., Search for relativistic magnetic monopoles with the Baikal neutrino telescope, Astropart. Phys. 29 (2008) 366.

[250] IceCube Coll., R. Abbasi et al., Search for relativistic magnetic monopoles with the AMANDA-II neutrino telescope, Eur. Phys. J. C 69 (2010) 361.

[251] ANTARES Coll., S. Adrián-Martínez et al., Search for relativistic magnetic monopoles with the ANTARES neutrino telescope (2011). arXiv:1110.2656 [astro-ph.HE].

[252] IceCube Coll., Search for relativistic monopoles with IceCube, to be subm. to Phys. Rev. Lett. (2011).

[253] M.S. Turner, E.N. Parker and T.J. Bogdan, Magnetic monopoles and the survival of Galactic magnetic fields, Phys. Rev. D 26 (1982) 1296.

[254] G. Giacomelli, S. Manzoor, E. Medinaceli and L. Patrizii, Searches for magnetic monopoles, nuclearites and Q-balls, J. Phys. Conf. Ser. 116 (2008) 012005. arXiv:hep-ex/0702050.

[255] I.A. Belolpatikov et al., The experimental limits on Q-ball flux with the Baikal deep underwater array 'Gyrlyanda' (1998). arXiv: astro-ph/9802223.

[256] I. Sokalski for the Baikal Coll., Search for magnetic monopoles with deep underwater Cherenkov detectors at Lake Baikal (1995). arXiv:9601160.

[257] A. Pohl, Search for subrelativistic particles with the AMANDA neutrino telescope, Ph.D. thesis, University of Uppsala, Sweden (2009). Available from: http://wwwiexp.desy.de/groups/astroparticle/pubs/Thesis.Arvid.090210.pdf.

[258] AMANDA and SPASE Coll., J. Ahrens et al., Measurement of the cosmic ray composition at the knee with the SPASE-2/AMANDA-B10 detectors, Astropart. Phys. 21 (2004) 565.

[259] AMANDA and SPASE Coll., J. Ahrens et al., Calibration and survey of AMANDA with the SPASE detectors, Nucl. Inst. Meth. A 522 (2004) 347.

[260] D.J. Boersma, L. Gladsstone and A. Karle for the IceCube Coll., Moon shadow observation by IceCube, contribution to 31st Int. Cosmic Ray Conf., Łódź, Poland (2009). Available from: http://icrc2009.uni.lodz.pl/proc/pdf/icrc1173.pdf, arXiv:1002.4900 [astro-ph.HE]

[261] ANTARES Coll., J.A. Aguilar et al., Zenith distribution and flux of atmospheric muons measured with the 5-line ANTARES detector, Astropart. Phys. 34 (2010) 179. arXiv: 1007.1777 [astro-ph.HE],

[262] E.V. Bugaev et al., Atmospheric muon flux at sea level, underground and underwater, Phys. Rev. D 58 (1998) 054001. arXiv:hep-ph/9803488.

[263] MILAGRO Coll., A.A. Abdo et al., The large-scale cosmic-ray anisotropy as observed with Milagro, Astrophys. J. 698 (2009) 2121. arXiv:0806.2293 [astro-ph]

[264] Tibet-AS $\gamma$ Coll., M. Amenomori et al., Anisotropy and corotation of Galactic cosmic rays, Science 314 (2006) 439. arXiv:astro-ph/0610671.

[265] S. Vernetto, Z. Guglielmotto and J.L.Zhang for the ARGO-YBJ Coll., Sky monitoring with ARGO-YBJ, contribution to 31st Int. Cosmic Ray Conf., Łódź, Poland (2009). Available from: 
http://icrc2009.uni.lodz.pl/proc/pdf/icrc0399.pdf, arXiv:0907.4615[astro-ph.HE]

[266] Super-Kamiokande Coll., G. Guillian et al., Observation of the anisotropy of 10 TeV primary cosmic ray nuclei flux with the Super-Kamiokande-I detector, Phys. Rev. D 75 (2007) 062003. arXiv:astro-ph/0508468.

[267] A.H. Compton and I.A. Getting, An apparent effect of Galactic rotation on the intensity of cosmic rays, Phys. Rev. 47 (1935) 817.

[268] IceCube Coll., R. Abbasi et al., Measurement of the anisotropy of cosmic ray arrival directions with IceCube, Astrophys. J. 718 (2010) L194. arXiv:1005.2960 [astro-ph.HE].

[269] IceCube Coll., R. Abbasi et al., Observation of anisotropy in the arrival directions of Galactic cosmic rays at multiple angular scales with IceCube. arXiv:1105.2326 [astro-ph.HE].

[270] L. Drury and F. Aharonian, The puzzling MILAGRO hot spots, Astropart. Phys. 29 (2008) 420. arXiv:0802.4403 [astro-ph].

[271] A. Lazarian and P. Desiati, Magnetic reconnection as the cause of cosmic ray excess from the heliospheric tail, Astrophys. J. 722 (2010) 188. arXiv:1008.1981 [astro-ph.CO].

[272] Pierre Auger Observatory homepage. Available from: http://www.auger.org.

[273] M.Sasaki, Y. Asaoka and M. Jobashi, Detecting very high energy neutrinos by the Telescope Array, Astropart. Phys. 19 (2003) 37. arXiv:astro-ph/0204167.

[274] D. Fargion, Discovering ultra-high-energy neutrinos by horizontal and upward $\tau$ air-showers: Evidences in terrestrial gamma flashes?, Astrophys. J. 570 (2002) 909. arXiv:astro-ph/0002453.

[275] D. Fargion, P.G. De Sanctis Lucentini and M. De Santis, Tau air showers from Earth, Astrophys. J. 613 (2004) 1285. arXiv:hep-ph/0305128

[276] Pierre Auger Coll., J. Abraham et al., Upper limit on the diffuse flux of UHE tau neutrinos from the Pierre Auger Observatory, Phys. Rev. Lett. 100 (2008) 211101. arXiv:0712.1909[astro-ph].

[277] EUSO Coll., A. Petrolini et al., The Extreme Universe Space Observatory (EUSO) instrument, Nucl. Phys. Proc. Suppl. 113 (2002) 329.

[278] JEM-EUSO homepage. Available from: http://jemeuso.riken.jp/en/index.html.

[279] F. Kajino for the JEM-EUSO Coll., The JEM-EUSO mission to explore the extreme universe, Nucl. Inst. Meth. A 623 (2010) 422, contribution to 1st Int. Conf. on Technology and Instrum. in Part. Phys. (TIPP09), Tsukuba, Japan.

[280] D. Fargion, Highest energy neutrino showers in EUSO (2002). arXiv:astro-ph/0212342.

[281] K. Shinozaki for the JEM-EUSO Coll., Extreme energy gamma rays and neutrinos and their observation in JEM-EUSO mission, AIP Conf. Proc. 1238 (2010) 377, contribution to 7th Tours Symp. on Nucl. Phys. and Astrophys., Kobe, Japan.

[282] K. Kotera, D. Allard and A.V. Olinto, Cosmogenic neutrinos: Parameter space and detectabilty from PeV to ZeV, J. Cosm. Astropart. Phys. 1010 (2010) 013. arXiv:1009.1382 [astro-ph.HE].

[283] K. Asano, K. Shinozaki and M. Teshima for the JEM-EUSO Coll., Sensitivity of JEM-EUSO to GRB neutrinos, contribution to 31st Int. Cosmic Ray Conf., Łódź, Poland (2009). Available from: http://icrc2009.uni.lodz.pl/proc/pdf/icrc0692.pdf, arXiv:0908.0392 [astro-ph.HE]

[284] G.A.Askaryan, Excess negative charge of an electron-photon shower and its coherent radio emission, Sov. Phys. JETP 14 (1962) 441.

[285] D. Saltzberg et al., Observation of the Askaryan effect: Coherent microwave Cherenkov emission from charge asymmetry in high energy particle cascades, Phys. Rev. Lett. 86 (2001) 2802. arXiv: hep-ex/0011001.

[286] P.W. Gorham et al., Observations of the Askaryan effect in ice, Phys. Rev. Lett. 99 (2007) 171101. arXiv:hep-ex/0611008.

[287] B. Price, Comparison of optical, radio, and acoustical detectors for ultrahigh-energy neutrinos, Astropart. Phys. 5 (1996) 43. arXiv:astro-ph/9510119.

[288] I. Kravchenko et al., RICE limits on the diffuse ultra-high energy neutrino flux, Phys. Rev. D 73 (2006) 082002. arXiv:astro-ph/0601148.

[289] ANITA Coll., S. Barwick et al., Constraints on cosmic neutrino fluxes from the ANITA experiment, Phys. Rev. Lett. 96 (2006) 171101. arXiv:astro-ph/0512265. 
[290] S. Barwick, ARIANNA: A new concept for UHE neutrino detection, J. Phys. Conf. Ser. 60 (2007) 278. arXiv:astro-ph/0610631.

[291] ARA Coll., P. Allison et al., Design and initial performance of the Askaryan Radio Array prototype EeV neutrino detector at the South Pole, subm. to Astropart. Phys.

arXiv: 1105.2854 [astro-ph.IM]

[292] P.W. Gorham et al., Experimental limit on the cosmic diffuse ultrahigh energy neutrino flux, Phys. Rev. Lett. 93 (2004) 041101. arXiv:astro-ph/0310232.

[293] O. Scholten et al., Improved flux limits for neutrinos with energies above $10^{22} \mathrm{eV}$ from observations with the Westerbork Synthesis Radio Telescope, Phys. Rev. Lett. 103 (2009) 191301. arXiv:0910.4745[astro-ph.HE].

[294] A. Horneffer et al., Cosmic ray and neutrino measurements with LOFAR, Nucl. Inst. Meth. A 617 (2010) 482.

[295] G.A. Askaryan, Hydrodynamic radiation from the tracks of ionizing particles in stable liquids, Sov. J. Atom. Energy 3 (1957) 921.

[296] J.G. Learned, Acoustic radiation by charged atomic particles in liquids: An analysis, Phys. Rev. D 19 (1979) 3293.

[297] IceCube Coll., R. Abbasi et al., Measurement of sound speed vs. depth in South Pole ice for neutrino astronomy, Astropart. Phys. 33 (2010) 277. arXiv:0909.2629 [astro-ph.IM]

[298] IceCube Coll., R. Abbasi et al., Measurement of acoustic attenuation in South Pole ice, Astropart. Phys. 34 (2010) 382. arXiv:1004.1694 [astro-ph.IM].

[299] IceCube Coll., R. Abbasi et al., Background studies for acoustic neutrino detection at the South Pole (2011). arXiv: 1103.1216 [astro-ph.IM].

[300] R. Nahnhauer, A.A. Rostovtsev and D. Tosi, Permafrost - An alternative target material for ultra-high energy neutrino detection?, Nucl. Inst. Meth. A 587 (2008) 29. arXiv:0707.3757 [astro-ph].

[301] R. Nahnhauer and S. Boeser (Eds.), Proc. 1st Int. Workshop on Acoustic and Radio EeV Neutrino detection Activities (ARENA), Zeuthen, Germany, Int. J. Mod. Phys. A, World Scientific, Singapore, 2006, ISBN 978-981-256-755-0.

[302] L. Thompson and S. Danaher (Eds.), Proc. 2nd Int. Workshop on Acoustic and Radio EeV Neutrino detection Activities (ARENA 2006), Northumbria, United Kingdom, Vol. 81 of J. Phys.: Conf. Ser., Institute of Physics Publ., Bristol, United Kingdom, 2007, ISBN 978-160-560-262-2.

[303] F. Ameli et al. (Ed.), Proc. 3rd Int. Workshop on Acoustic and Radio EeV Neutrino detection Activities (ARENA 2006), Rome, Italy, Vol. A 604 of Nucl. Inst. Meth., Elsevier, 2009.

[304] N. Lehtinen et al., Sensitivity of an underwater acoustic array to ultra-high energy neutrinos, Astropart. Phys. 17 (2002) 279. arXiv:astro-ph/0104033

[305] J. Vandenbroucke, G. Gratta and N. Lehtinen, Experimental study of acoustic ultrahigh-energy neutrino detection, Astrophys. J. 621 (2005) 301. arXiv:astro-ph/0406105.

[306] SAUND II Coll., N. Kurahashi et al., Study of acoustic ultra-high energy neutrino detection phase II, Int. J. Mod. Phys. A 21 S 1 (2006) 217.

[307] ANTARES Coll., J.A. Aguilar et al., AMADEUS - The acoustic neutrino detection test system of the ANTARES deep-sea neutrino telescope (2010). arXiv:1009.4179 [astro-ph.IM] 